NASACONTRACTOR REPOR T

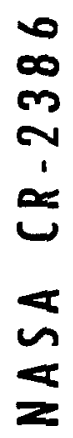

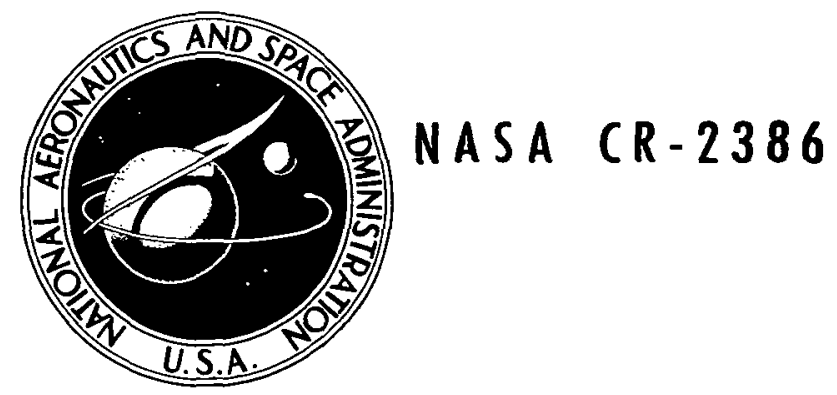

\title{
A STUDY OF SURFACE PRESSURE FLUCTUATIONS IN HYPERSONIC TURBULENT BOUNDARY LAYERS
}

by K. R. Raman

Prepared by

NIELSEN ENGINEERING \& RESEARCH, INC.

Mountain View, Calif. 94043

for Ames Researcb Center

NATIONAL AERONAUtics AND SPACE ADMINISTRATION - WASHINGTON, D. C. - FEBRUARY 1974 


\begin{tabular}{|c|c|c|c|}
\hline $\begin{array}{l}\text { 1. Report No. } \\
\text { NASA CR }-2386\end{array}$ & 2. Government Accession No. & \multicolumn{2}{|c|}{ 3. Recipient's Catalog No. } \\
\hline \multirow{2}{*}{$\begin{array}{l}\text { 4. Title and Subtitle } \\
\text { "Surface Pressure Fluctuations i } \\
\text { Layers" }\end{array}$} & \multirow{2}{*}{ in Hypersonic Turbulent Boundary } & \multicolumn{2}{|c|}{$\begin{array}{l}\text { 5. Report Date } \\
\text { February } 1974\end{array}$} \\
\hline & & \multicolumn{2}{|c|}{ 6. Performing Organization Code } \\
\hline \multirow{2}{*}{$\begin{array}{l}\text { 7. Author(s) } \\
\text { K. R. Raman }\end{array}$} & & \multicolumn{2}{|c|}{ 8. Performing Organization Report No. } \\
\hline & & \multirow[t]{2}{*}{ 10. Work Unit No. } & . \\
\hline \multirow{3}{*}{$\begin{array}{l}\text { 9. Performing Organization Name and Address } \\
\text { Nielsen Engineering \& Research; } \\
\text { Mountain View, California } 94043\end{array}$} & \multirow{3}{*}{ Inc. } & & \\
\hline & & \multicolumn{2}{|c|}{$\begin{array}{l}\text { 11. Contract or Grant No. } \\
\text { NAS } 2-6838\end{array}$} \\
\hline & & \multirow{2}{*}{\multicolumn{2}{|c|}{$\begin{array}{l}\text { 13. Type of Report and Period Covered } \\
\text { Final Report }\end{array}$}} \\
\hline \multirow{2}{*}{\multicolumn{2}{|c|}{$\begin{array}{l}\text { 12. Sponsoring Agency Name and Address } \\
\text { National Aeronautics \& Space Administration } \\
\text { Washington, D.C. } 20546\end{array}$}} & & \\
\hline & & \multicolumn{2}{|c|}{ 14. Sponsoring Agency Code } \\
\hline \multicolumn{4}{|l|}{ 15. Supplementary Notes } \\
\hline \multicolumn{4}{|c|}{$\begin{array}{l}\text { 16. Abstract } \\
\text { The surface pressure fluctuations on a flat-plate model at hyper } \\
5.2,7.4 \text { and } 10.4 \text { with an attached turbulent boundary layer were } \\
\text { mounted small piezoelectric sensors. A high frequency resolutio } \\
\text { was achieved using specially designed small piesoelectric sensor } \\
\text { had a good frequency response well above } 300 \mathrm{KHz} \text {. The RMS press } \\
\text { energy spectra for alI above Mach numbers are presented. The co } \\
\text { obtained from space-time correlation considerations are equal to } \\
\text { indicate the RMS pressures vary from } 5 \text { to } 25 \text { percent of the mean } \\
\text { ratios of RMS pressure to dynamic pressure are less than the uni } \\
\text { subsonic value of } 6 \times 10^{-3} \text {. The ratio decreases in value as the } M \\
\text { pressure is increased. The ratio of RMS pressure to wall shear } \\
\text { satisfies } 1 \leq \mathrm{p} / \tau_{\mathrm{w}} \leq 3 \text {. Th }\end{array}$} \\
\hline $\begin{array}{l}\text { 17. Key Words (Suggested by Author(s)) } \\
\text { Fluctuating Pressures } \\
\text { Boundary Layer Turbulence } \\
\text { Hypersonic Flow } \\
\text { Aerodynamic Noise }\end{array}$ & 18. Distribution Stat & IFIED-UNLIMITED & Cat.12 \\
\hline $\begin{array}{l}\text { 19. Security Classif. (of this report) } \\
\text { UNCLASSIFIED }\end{array}$ & $\begin{array}{l}\text { 20. Security Classif. (of this page) } \\
\text { UNCLASSIF IED }\end{array}$ & $\begin{array}{l}\text { 21. No. of Pages } \\
90\end{array}$ & $\begin{array}{l}\text { 22. Price } \\
\$ 3.75\end{array}$ \\
\hline
\end{tabular}

* For sale by the National Technical Information Service, Springfield, Virginia 22151 
TABLE OF CONTENTS

Page

No.

SUMMARY

INTRODUCTION.

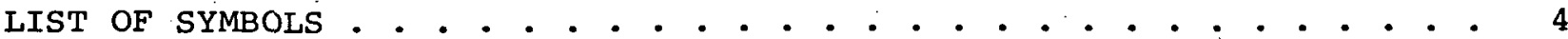

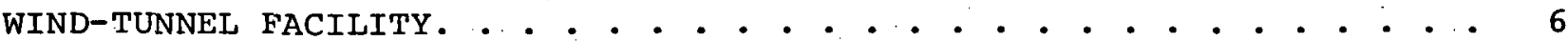

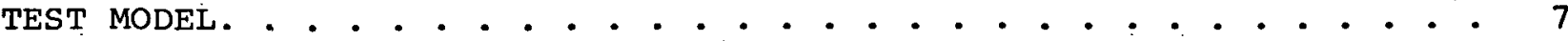

INSTRUMENT PLUG . . . . . . . . . . . . . . . . . . . . . . . 8

INSTRUMENTATION • . . . . . . . . . . . . . . . . . . . . . . . . . 10

Data Acquisition/Retreival System. . . . . . . . . . . . . 10

Shock Tube Calibration Apparatus . . . . . . . . . . . . . . . 10

Pressure Sensors . . . . . . . . . . . . . . . . . . . . . 11

Amplifiers . . . . . . . . . . ... . . . . . . . . . . . 14

Accelerometer. . . . . . . . . . . . . . . . . 16

Temperature Sensor ... . . . . . . . . . . . . . . . . 16

Correlator . . . . . . . . . . . . . . . . . . . . 17

Spectrum Analyzer. . . . . . . . . . . . . . . . . . . . . 17

Root-Mean-Square Meter . . . . . . . . . . . . . . . . . 18

TEST PROCEDURE. . . . . . . . . . . . . . . . . . . . . . . . . 18

DATA ANALYSIS . . . . . . . . . . . . . . . . . . . . . . . . . . 19

MEAN AERODYNAMIC BOUNDARY-LAYER PARAMETERS. . . . . . • • . . . . . . . 20

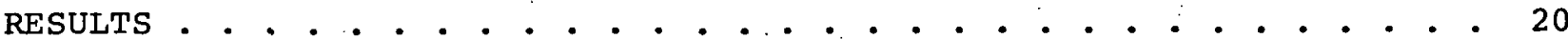

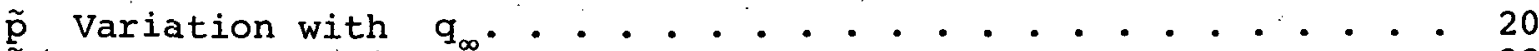

$\tilde{\mathrm{p}} / \mathrm{p}$ Variation with $\mathrm{q}_{\infty} \cdot$. . . . . . . . . . . . . . . . . 22

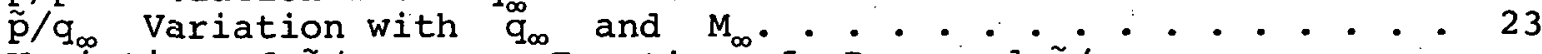

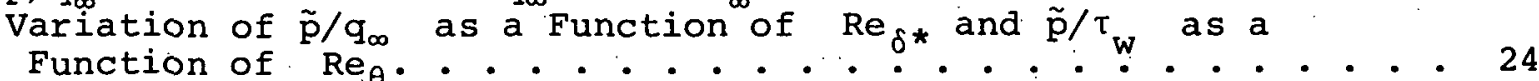

Spectral Considerations. . . . . . . . . . . . . . . . . 25

Correlation Functions. . . . . . . . . . . . . . . . . . 28

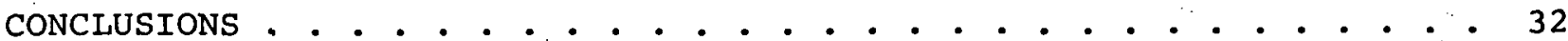

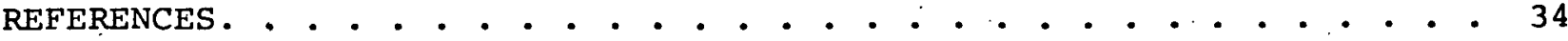

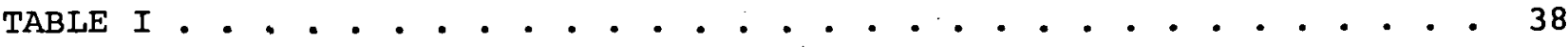

FIGURES 1 THROUGH 39. . . . . . . . . . . . . . . . . . . . . 


\section{A STUDY OF SURFACE PRESSURE FLUCTUATIONS}

IN HYPERSONIC TURBULENT BOUNDARY LAYERS

By K. R. Raman

Nielsen Engineering \& Research, Inc.

\section{SUMMARY}

An experimental investigation of the surface pressure fluctuations on a flat-plate model at hypersonic speeds of $5.2,7.4$ and 10.4 with an attached turbulent boundary layer was carried out at Ames Research Center. The statistical data obtained include RMS pressures and nondimensionalized energy spectra for all the three Mach numbers. Space-time correlations and the convective velocities were obtained for tests at Mach number of 7.4 only. High frequency resolution of the pressure field was achieved using specially designed small piezoelectric sensors (diameter $=0.5 \mathrm{~mm}$ ) that had a frequency response well above $300 \mathrm{KHz}$. The results indicate the RMS pressures vary from 5 to 25 percent of the mean static pressures. The ratio of RMS pressures to dynamic pressures is less than the universally accepted subsonic value of $6 \times 10^{-3}$. The ratio decreases in value as the Mach number or the dynamic pressure is increased. The ratio of RMS pressure to wall shear stress for $M_{\infty}=7.4$ satisfies $1 \leq \tilde{p} / \tau_{w} \leq 3$. The broad-band space-time correlations with different streamwise spatial separations are characterized by a convective velocity $\mathrm{U}_{\mathrm{C}}$ nearly equal to 0.7 of the free-stream velocity.

\section{INTRODUCTION}

The generation of sound is in general due to vibrations of surfaces or fluctuating shear flows in a compressible medium. These vibrations produce a fluctuating force in the medium and cause density and pressure fluctuations that radiate energy outward. If the intensity and frequency of these pressure fluctuations fall in the audible region we perceive them as sound. Knowledge of the pressure fluctuations on the surface within a turbulent boundary layer at various Mach numbers is essential in many problems. Although considerable work has been performed to investigate such fluctuations at subsonic and supersonic speeds, little information is 
availalbe at hypersonic flows. At subsonic and supersonic speeds and at large Reynolds numbers, a major portion of the exterior surface of flight vehicles is subjected to pressure fluctuations due to attached and/or separated turbulent flow. The intensity of these fluctuating pressures and their frequency components $(\leq 300 \mathrm{KHz}$ expected at hypersonic free-stream velocities) could significantly influence the dynamic load analysis of the structure and lead to fatigue of the vehicle.

The fatigue loads caused by the fluctuating acoustic pressures may determine design requirements for the skin of hypersonic re-entry vehicles, cruise vehicles, or logistic resupply space vehicles. The vibrations and noise transmitted through the structure can affect adversely the components in guidance instruments, decrease flight crew efficiency. and affect the protective thermal shield of space shuttle vehicles. For structural dynamics and analysis, the designer is interested in being able to predict pressure data related to the aerodynamic parameters of the turbulent external flow over the surface of the vehicle at hypersonic speeds. These fluctuating pressure data enable one to assess the intensity of a turbulencegenerated sound field that radiates its energy from sources in the boundarylayer region. For a successful suppression of the unwanted noise thus generated, it would be necessary to understand the source characteristics.

The noise produced by the fluctuating pressure field in a turbulent boundary layer can be classified into "far" and "near" fields. The turbulent fluctuations will (i) radiate a sound field into the external flow, the "far" field and (ii) create pressure and shear fluctuations adjacent to the surface, the "near" field. If the bounding surface is flexible, coupling exists between the wall motion and the external flow field.

A literature survey on the fluctuating pressure field in turbulent boundary layers reveals theoretical treatments of the problem by philips (refs. I and 2), Curle (ref. 3), Kraichnan (refs. 4-6), Lilley and Hodgson (ref: 7), and others. In addition, several experimental investigations concerning incompressible boundary layers in wind tunnels have been carried out by Harrison (ref. 8), Willmarth (refs. 9-14), Bull (refs. 15-17), and Serafini (ref. 18). Laufer (ref. 19 and 20) considered the supersonic far field problem. Chen (ref. 21), Kistler and Chen (ref. 22), Lilley (ref. 23), Speaker and Ailman (ref. 24), Murphy et al. (ref. 25) Maestrello (ref. 26), and coe and Chyu (ref. 27) carried out experiments in the turbulent boundary 
layer at supersonic speeds up to Mach number 3.5. The pressure field investigation of separated flow in the boundary layer due to a step or interacting oscillating incident shock wave has been performed by speaker and Ailman (ref. 24) and coe (refs. 27 and 28). In addition to all the above literature, there are some free flight data obtained from a glider flight, and from powered airplanes (refs. 29-35).

All the above literature indicates that the statistical pressure information for the incompressible, attached turbulent boundary layer is fairly complete. At transonic and supersonic speeds, measurements of the near field pressure intensity have been carried out; in addition there exists some spectral and correlation information for the attached supersonic flows. From these limited experimental results at supersonic speeds, it can be concluded that the data are consistent with the findings at subsunic range and that the information on the pressure field is well in hand. Beyond the supersonic range there is a scarcity of data and no direct pressure fluctuation measurements have so far been reported in the 1 iterature. Wallace (ref. 36) and Harvey et al. (ref. 37) have given some pressure intensity values at hypersonic velocities. These were obtained indirectly by calculations from temperature and density fluctuation data. These measurements were made with the use of an electron beam technique at hypersonic speed. The present investigation of near field pressure fluctuations at hypersonic speeds is one of the first studies that provide direct measurements.

The experimental research reported here is concerned with the problem of a fluctuating pressure field within an attached turbulent boundary layer over a flat-plate model when the free-stream velocities are in the hypersonic range $\left(5 \leq M_{\infty} \leq 10.4\right)$. The tests were carried out in the Ames Research Center 3.5 Foot Hypersonic Wind Tunnel, and the range of Reynolds numbers in the experiments was from 0.9 to 15 million.

A major part of the work reported here was carried out at Ames Research Center, Moffett Field, CA by the author as an employee of NASA prior to October 1971. The data analysis and preparation of the report were carried out at Nielsen Engineering \& Research, Inc., Mountain View, CA on Contract No. NAS2-6838 from Ames Research Center. 
A

$c_{f}$

d

f

$f_{L F}$

$\mathrm{f}_{\mathrm{HF}}$

g

$G(f)$

$H(\omega)$

$M_{\infty}$

$\mathrm{M}_{\mathbf{S}}$

$p(\vec{x}, t)$

$p^{2}(\vec{x}, f)$

$\overline{p^{2}(\vec{x})}$

$\tilde{p}$

$\mathrm{P}_{\mathrm{R}}$

$\Delta \mathrm{p}$

$q_{\infty}$

R

$\operatorname{Re}$

$R_{i i}(\vec{x}, \tau)$ constant related to ratio of RMS pressure to dynamic pressure see equation (13)

local skin-friction coefficient

diameter of sensor, meters

frequency, $\mathrm{Hz}$

low frequency roll-off setting, $\mathrm{Hz}$

high frequency roll-off setting, $\mathrm{Hz}$

gravitational acceleration, $980 \mathrm{~cm} / \mathrm{sec}^{2}$

nondimensional power spectral density function of $p(t)=p^{2}(f) U_{\infty} / q_{\infty}^{2} \delta^{*}$, see equation (10)

transfer function of sensor in frequency domain

free-stream Mach number

shock wave speed

static pressure, $\mathrm{N} / \mathrm{m}^{2}$

energy or power spectral density function of $\mathrm{p}(\mathrm{t}),\left(\mathrm{N} / \mathrm{m}^{2}\right)^{2} / \mathrm{Hz}$

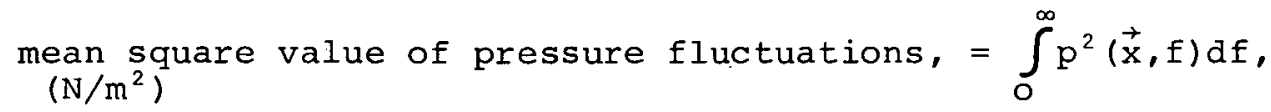

root-mean-square (RMS) value of pressure fluctuations,

$\sqrt{\overline{\mathrm{p}^{2}(\overrightarrow{\mathrm{x}})}}, \mathrm{N} / \mathrm{m}^{2}$

reservoir pressure, $\mathrm{N} / \mathrm{m}^{2}$

pressure step obtained in the shock tube calibration apparatus, $\mathrm{N} / \mathrm{m}^{2}$

free-stream dynamic pressure, $\mathrm{N} / \mathrm{m}^{2}$

percent variation in Reynolds number, see equation (6)

Reynolds number

normalized auto-correclation function, $R_{i i}(\vec{x}, \tau)=$ $\overline{p_{i}(\vec{x}, t) p_{i}(\vec{x}, t+\tau)} / \tilde{p}_{i}^{2}$ 


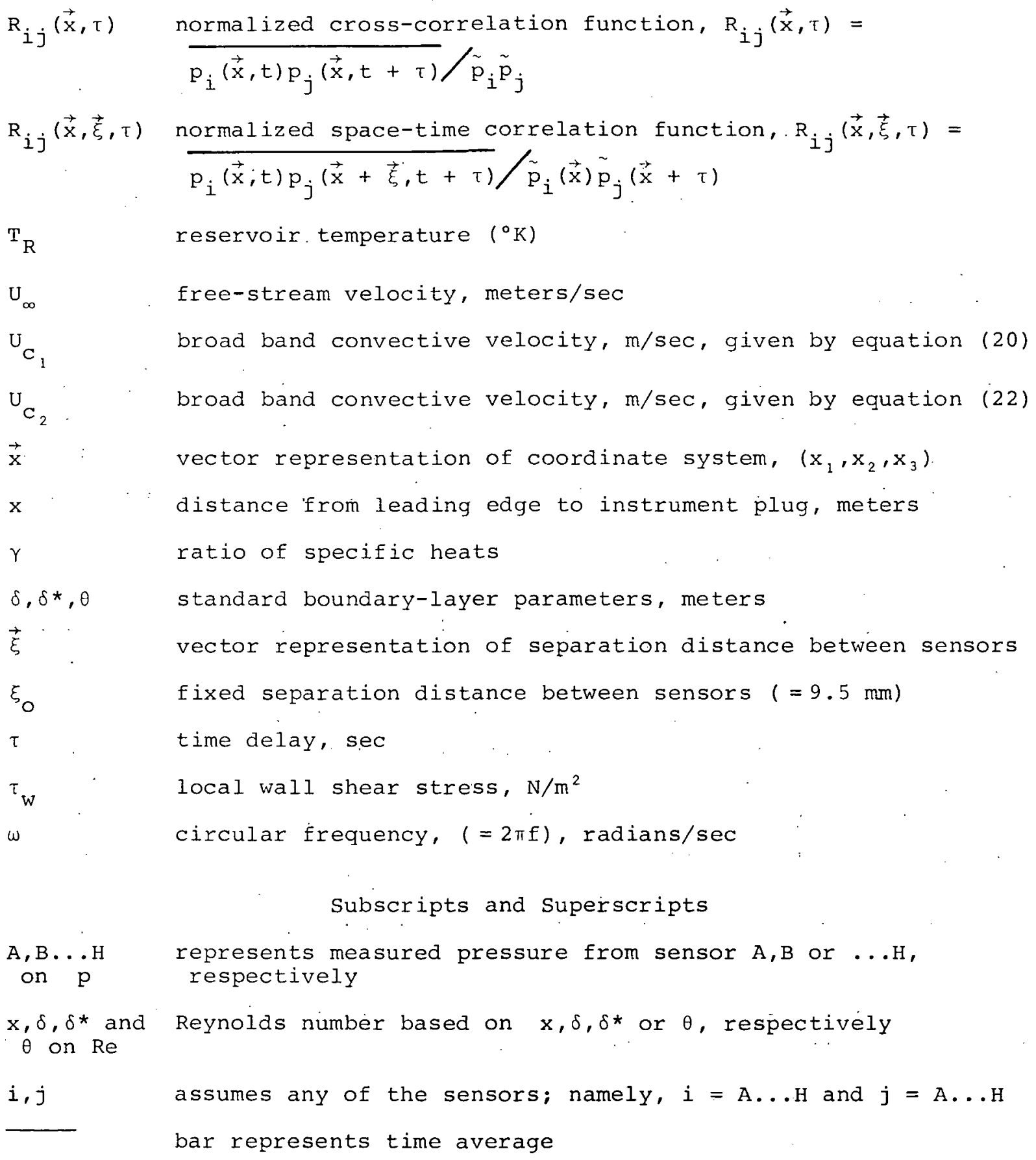




\section{WIND-TUNNEL FACILITY}

The tests for this investigation were carried out in the Ames 3.5-Foot Hypersonic Wind Tunnel. Schematic drawings of the facility are given in figure $l(a)$ and (b). Air, free of dust and water vapor, from high pressure tanks passes through a heated alumina pebble bed chamber, an interchangeable nozzle section, a test section and a diffuser portion of the tunnel prior to reaching large evacuated spheres. From thesé spheres the air is returned to the high pressure tanks. The air is preheated by the pebble heat exchanger in order to prevent liquefaction in the test section.

Because of the large frictional losses due to large velocity gradients at the interior wall surfaces, small amounts of helium are injected tangentially to the interior surface at the throat of the nozzle section. This reduces the power required for the wind-tunnel operation and increases the useful core cross section of uniform test stream and substantially maintains the tunnel walls at a low temperature. After each test run the air is cleaned to remove dust and moisture, and the helium is separated by a cryogenic separation process. The air is then returned to the high pressure: storage tanks.

The wind tunnel is capable of maintaining a continuous steady flow in the test section for any of the preselected nozzle configurations at hypersonic speeds for a maximum duration of 5 minutes. For each preselected test stream Mach number of $5.2,7.4$ or 10.4, the appropriate nozzle configuration must be installed between the pebble bed heater and the test section. The wind-tunnel temperature and pressures were monitored both in the reservoir (in the pebble bed itself) and at several locations in the test section.

The tunnel conditions for each of the tests are given in Table I. In general the tests spanned the following conditions:

\footnotetext{
$5.2 \leq$ Mach number, $M_{\infty} \leq 10.4$

$1 / 2 \leq$ duration of test run, minutes $\leq 1$

$6.4 \times 10^{5} \leq$ reservoir pressure, $\mathrm{N} / \mathrm{m}^{2} \leq 1.10 \times 10^{7}$

$700 \leq$ reservoir temperature, ${ }^{\circ} \mathrm{K} \leq 1200$
} 


$$
\begin{aligned}
6.8 \times 10^{3} & \leq \text { dynamic pressure, } \mathrm{N} / \mathrm{m}^{2} \leq 6.6 \times 10^{4} \\
9 \times 10^{5} & \leq \text { Reynolds number, Re } \mathrm{Re} \leq 1.45 \times 10^{7}
\end{aligned}
$$

The model was injected into the test stream once the preselected conditions were reached in the wind tunnel. The model injector mechanism, shown in figure 2 , is operated hydraulically. The injector mechanism permitted retention of the test model in the stream for a minute or less, the time required for data acquisition on a high speed tape recorder. When the quick insert mechanism is activated, the side wall of the tunnel slides out. The model mounted on the quick insert arm is inserted into the middle of the test section at the predetermined angle of attack and the sive wall closes to prevent the hot test stream from flowing into the quick insert chamber. After the data are taken, the model is retracted and the exact reverse of the above described process takes place.

In cases of emergency, the model can be retracted quickly by a built-in alternative system combining a spring and explosive charge cartridge. At a Mach number of 5.2 and high dynamic pressures, this alternative system was used.

\section{TEST MODEL}

The model used in these tests, figure 3 , was a $1.9-\mathrm{cm}$ thick aluminum plate, $120 \mathrm{~cm}$ long and $45 \mathrm{~cm}$ wide. The fine dust from the disintegrating alumina pebbles in the heater impinged on the leading edge. The windtunnel dust particles were not a major problem within the boundary layer: A hard Inconel leading edge with a nose radius of $0.05 \mathrm{~mm}$ and internal water cooling to withstand the high stagnation temperature was used in the tests. The entire surface was honed and lapped to provide a highly polished smooth surface. The surface was anodized in order to prevent rough irregular surface oxidization due to moisture from ambient air.

The plate was shock mounted on the injector mechanism to prevent any gross extraneous wind-tunnel shell, or injector unit vibrations from affecting the measurements. The photographs of the test model in figures 3 and 4 show front, side and back views of the model with all the items described above identified. 
The plate was provided with two holes of $5.7 \mathrm{~cm}$ diameter along the centerline, about $73 \mathrm{~cm}$ and $84 \mathrm{~cm}$ downstream of the leading edge to accept the instrument plugs. Underneath the smooth test surface was a completely enclosed chamber for the instruments to protect them from the heated air and high stream velocities (fig. 4). The electrical wires from all the instruments passed through a double-walled, water-cooled conduit from the instrument box and emerged from the injector unit described in the wind-tunnel section.

In order to protect the flow on the smooth side of the plate from any spill-over from the underside flow conditions, rigid skirts were provided along the entire length of the plate on each side, figure 3. This also insured a two-dimensional mean flow condition at the instrument plug.

\section{INSTRUMENT PLUG}

The instrumentation was concentrated in a plug mounted in the flat plate. The instrument plug is a circular $5.7 \mathrm{~cm}$ diameter and $1.9 \mathrm{~cm}$ thick disk which could be installed flush with the plate into either hole along the longitudinal axis of the test model. For the present tests the instrument was installed $73 \mathrm{~cm}$ from the leading edge. A noninstrument insert was installed at the second hole, $83 \mathrm{~cm}$ from the leading edge. The disk with various instruments was shock mounted using o'rings as shown in figure 5 in order to eliminate mechanical vibrations and acceleration effects. In the photograph of the fully instrumented plug the sensors are labeled from $A$ to $\mathrm{H}$ and the two steady-state static pressure sensors are labeled $I$ and II. The sensors are located $9.5 \mathrm{~mm}$ apart center to center in both longitudinal and lateral directions.

Each of these sensors was carefully mounted under a binocular microscope, and the annular gap (less than $0.02 \mathrm{~mm}$ ) between the sensor and the instrument plug was filled with silicone rubber to avoid any presence of Helmholtz resonance and the accompanying discrete frequency sound. The rubber could withstand high'temperatures without appreciable swelling or contraction. This precaution was essential in several respects: it avoided any roughness causing local stream perturbations and it eliminated local effects of Helmholtz resonance and acted as a shock mounting for the sensor. 
The back side of the instrument plug is shown in figure 6 . Various sensors used for pressure and temperature monitoring and the sourcefollower amplifiers are pointed out. The steady-static pressure reference was obtained from the two static pressure orifices by connecting each orifice to a coil of 3.5 meters long hypodermic țubing with a $0.5 \mathrm{~mm}$ inner diameter. The attenuation of the fluctuating pressure components in long, small diameter tubes is well known (refs. 38 and 39) and the pressures so obtained were fed to a common manifold located on the back side of the instrument plug. The manifold supplied the required reference pressure for all the diaphragm sensors. The steady-static pressures (prior to common manifold) were recorded on tape during the tests. The reason for two separate steady-static pressures venting to the manifold was to avoid loss of data or sensor due to unexpected clogging of the orifice by dust particles.

The thermocouples used were made by thin-film technique and the thermocouple elements were chromel-constantum. Because there was very little mass, the thermocouples were good for high frequency measurements. The thermocouples $\mathrm{T}_{1}$ to $\mathrm{T}_{4}$ shown in figure 5 were carefully mounted on a kel-F heat insulator to avoid the instrument plug acting as a heat sink during the data acquisition period. One temperature sensor ( $\mathrm{T}_{5}$ in figure 5 ) was mounted directly on the plate to monitor the temperature rise during the tests.

The sensors mounted on the instrument plug are listed below:

(1) 4 crystal sensors ( $1 \mathrm{KHz} \leq$ freq $\leq 500 \mathrm{KHz}$ )

(2) 4 diaphragm sensors $(0.3 \mathrm{KHz} \leq$ freq $\leq 80 \mathrm{KHz})$

(3) 4 thin-film thermocouples mounted on Kel-F heat insulators

(4) I thin-film thermocouple to sense the plate temperature during tests

(5) 2 static pressure orifices (steady-state pressures)

(6) 1 accelerometer

(7) 1 reference manifold 


\section{INSTRUMENTATION}

In these tests several sensors were used with their associated electronic amplifiers to obtain signal levels sufficient for data acquisition on an analog tape recorder system. The parameters measured were the surface static pressure time history at several locations, the acceleration of the test model, the surface temperature and the static and total pressure and total temperature in the test section. The data acquisition/retrieval system, the amplifiers, and various pressure, temperature and accelerometer sensors used will be discussed next.

\section{Data Acquisition/Retrieval System}

A specially designed 20-channel analog magnetic tape record/reproduce system made by Ampex (Model 1800H) was used with a recording tape speed of 3.048 meters/second. The recorder has nine channels of wide band direct $(400 \mathrm{~Hz}-1.5 \mathrm{MHz}$ ) record-reproduce and nine channels of FM (DC - $40 \mathrm{KHz}$ ) record/reproduce modes of operational capability. In addition two channels were used for time-code and tape control. An edge track voice channel was also available. All necessary additional electronics for reproduce phase at two additional speeds of 0.381 and 0.048 meters/second were provided also.

A one-megahertz tape speed reference oscillator signal was recorded on a direct data track and was useful in assessing any tape stretch problem and in determining dynamic skewness between two adjacent channels. This also provided a means of obtaining information on the cross-talk between channels. The record and reproduce operations are bi-directional at all the selected speeds. The tape recorder can shuttle between any two preselected start and stop time code information off the recorded data, which is useful in the data analysis phase.

The Time Code Generator (Model 6190), Time Code Translator (Model 5220) and Tape Search and Control (Model 5224) were all made by Astrodata. These units were integrated into the tape recorder system as a single unit.

Shock Tube Calibration Apparatus

A shock tube was built for static and dynamic calibration of pressure sensors and is shown in figure 7. When the aluminum foil diaphragm is 
ruptured, the atmospheric pressure generates a one-dimensional shock wave that travels down the $180 \mathrm{~cm}$ long, $5.7 \mathrm{~cm}$ diameter low pressure chamber. The shock speed is obtained by determining the lapse time between two sensors located $5.1 \mathrm{~cm}$ apart on the side wall of the low pressure chamber. The instrument plug is mounted at the end of the tube. Once the shock speed, $M_{S}$ and the ambient initial test conditions are known, the input pressure pulse experienced by the instruments can be obtained from normal shock relations (ref. 40). The shock tube, being portable, was easy to use at the wind-tunnel location for periodic calibration checks on the sensors. Further, the low pressure chamber could be brought against the test model and the suction in the chamber made a perfect seal between the shock tube and the model prior to diaphragm rupture. Thus, the entire bank of sensors could be checked without seriously disrupting the tests. The low pressure chamber was evacuated to $280 \mathrm{~mm} \mathrm{Hg}$ before the $2.54 \times 10^{-3} \mathrm{~cm}$ thick aluminum foil failed. In all the calibration tests, the shock speed did not exceed $M_{s}=3.0$.

The static calibration of the diaphragm sensors was also accomplished with the shock tube apparatus. The low pressure chamber of the shock tube was evacuated in several steps and held at each pressure for the static sensor response to be recorded. For this calibration, the backside of the diaphragm sensors were vented to ambient atmospheric pressure while the manifold, venting to various sensors, was closed. The output of each diaphragm sensor was directly read (without the amplifiers in the circuit) through the use of a digital millivolt meter at each low pressure setting. This procedure was necessary since the Princeton amplifiers have a high pass filter setting of $10 \mathrm{~Hz}$ (the lowest operational frequency limit) and cannot register any DC voltages.

\section{Pressure Sensors}

A study of the statistical pressure characteristics of boundary-layer turbulence through spectral and correlation (space-time) considerations demands a very small sensor with a flat frequency response from low audio frequency to ultrasonic frequency. The subsonic and supersonic turbulent boundary-layer literature indicates that at hypersonic velocities, pressure fluctuations with acoustic energy of interest could be expected up to $300 \mathrm{KHz}$ (refs.2l and 22). Since no commercially available transducer with 
the required frequency response characteristics could be found, a piezoelectric sensor was designed and developed for this program (ref. 4l). A schematic drawing of the sensor is shown in figure 8 .

The basic element of the sensor is a Lead-zirconate-Titanate crystal. The unique design feature of the crystal sensor is the shock mounting of the sensing element. This is essential to avoid a signal being generated by mechanical vibration of the plate when the plate is inserted into the tunnel. Subsequent tests of the sensor mounted in the plate indicated negligible response even when hitting the plate with a mallet.

The piezoelectric material is ideally suited as an active transducer element. The desired qualities are (i) its high sensitivity and linearity to applied stresses, (ii) its very high natural frequency characteristics, (iii) its wide dynamic range of operation, (iv) its adaptability to subminiaturization, and $(v)$ its ready availability in disk or cylinder forms. In addition, present-day electronics make it possible to use the metal oxide semiconductor (MOS) and/or field effect transistor (FET) circuitry for the matching preamplifier (Emitter-follower or Cathode-follower circuit) with high input impedance and low output impedance. The use of these FET amplifiers directly connected to the sensor eliminated the usual parallel cable capacitance problem (due to long leads) that degrades the sensor's output. This approach also avoided the extraneous noise source generated by the motion of the connecting cable. The approach of directly connecting a small impedance matching electronics circuit eliminated the need for a special low noise connecting cable between the sensor and the FET amplifier. A comercially available integrated circuit source-follower amplifier (PCB Piezotronics Model 402All) was connected directly to the sensor in the tests (see fig. 6).

The choice of Titanium backing material for the piezoelectric sensing element provided almost perfect acoustic impedance matching. This is essential to minimize the interface interference effects. Further, the Titanium rod used in the sensor construction was brought to a point. This sharp point provided proper focusing of the incoming acoustic wave to progress along the backing material and dissipate its energy at the tip (ref. 42). Thus, the usual ringing phenomenon observed in sensors of this type was completely eliminated from the sensor used in these tests. 
In addition to the crystal pressure sensors, commercially available diaphragm-type Kulite sensors (CPL-070-4) with an overall diameter of 1.78 $\mathrm{mm}$ and a useful frequency range from zero to about $80 \mathrm{KHz}$ were used in these tests. These sensors in combination with the crystal sensors made it possible to obtain data from very low to very high frequencies. The Kulite sensor consists of a stretched thin diaphragm on which four piezoresistive strain gages are vacuum deposited to form a wheatstone bridge. The presence of all the elements on the diaphragm eliminates temperature compensation problems. The upper frequency range of the sensor is directly dependent on the resonant frequency of the stretched diaphragm.

The calibration curves for both Kulite and crystal sensors are given in figures 9(a), (b) and (c). The small shock tube (discussed previously) was used for obtaining both static and dynamic calibration data on these sensors. The solid symbols in these figures were obtained from dynamic tests and were usually lower than the static data by about 5 percent.* Similar results have been observed by Kendall (ref. 43). The crystal sensor calibration data were obtained by dynamic tests only. The calibration data. of all the sensors used in the tests are given in figure 10, in terms of accepted decibel terminology with reference sensitivity given as $1 \mathrm{~V} / \mu \mathrm{bar}$. In addition, the time-rise response of less than one microsecond of the crystal sensor was determined by its response to a sudden pressure step generated by the nose shock off a bullet (Super 225 winchester) in flight at Mach 3 that swept across the face of the transducer (fig. 11). From the time history of the input and the measured output of the sensor response one can determine through Fourier transform methods the transfer function for the sensor. The complex transfer function is given by

$$
\begin{aligned}
& H(\omega)= \text { Fourier transform of sensor response/Fourier } \\
& \text { transform of input pressure step }
\end{aligned}
$$

The frequency response of both amplitude and phase thus obtained from $\mathrm{H}(\omega)$ is given in figure 12 (ref. 44). Shadowgraph pictures of the flight of the bullet as the nose shock wave swept the sensor surface were obtained

\footnotetext{
*The difference between static and dynamic calibration data depends on various parameters, none of which have been systematically established to date. It is fortunate that in the present case the dynamic data fell systematically lower than the static data; and there is only a small percent error incurred in using static calibration data.
} 
in order to determine the pressure step, $\Delta \mathrm{p}$ imposed on the sensor. From these pictures the incident shock angle and the angle of reflection over the sensor's flat surface were determined. Using the known forward velocity of the bullet (equal to shock speed), the ambient atmospheric condition ahead of shock wave, the measured incident and reflected angles, the step pressure, $\Delta \mathrm{p}$ imposed on the sensor, was calculated. In the above a two-dimensional flow condition was assumed (curvature of the spreading shock cone was ignored) and tables from reference 40 were used. The sensitivity of the sensor obtained in this fashion was identical with the ones obtained from calibration with the shock tube.

To avoid prolonged exposure of the sensitive instruments to the heated air stream in the test section, the model was injected into the stream for a short time and then retracted (fig. 13). During the model's stay in the tunnel, data were recorded on magnetic tape at 3.048 meters/second tape speed. The surface wall temperatures of the model were measured at the instrument plug during the model's stay in the tunnel; and data for 30 seconds prior to model retraction from the stream are given in figure 14. The temperature rose from the ambient conditions by less than 10 percent of the breakdown Curie temperature of $360^{\circ} \mathrm{C}$ for PZT-5 material. The reservoir temperatures for these runs at the two Mach numbers given in the plots were around $1100^{\circ} \mathrm{C}$. The temperature drop immediately after the modél was retracted into the quick insert chamber is noticeable in the figure.

\section{Amplifiers}

Three different types of amplifiers were used in these tests. These amplifiers were selected for their compatibility with the sensor and the frequency range of interest of the parameter being measured.

The piezoelectric transducers, being self-generating charge devices, exhibit high source impedance to the signal conditioning electronics and are essentially all capacitance. Thus, the amplitude and phase characteristics will be direct functions of the load on the system. In order to utilize the excellent undamped high frequency response of the piezoelectric sensors and avoid adverse effects on their phase characteristics, the associated broad-band amplifier should possess matching high input impedance and low electrical (or Johnson) noise. The interconnecting cable 
lengths between the sensor and the matching amplifier should also be kept to a minimum in order to avoid degradation of the low frequency characteristics of the sensor.

The choice for the tests was a small integrated circuit sourcefollower amplifier (accomplishing the same functions as a cathode-follower or an emitter-follower) made by PCB Piezotronics, Inc. (Model 402Al1). The input resistance and capacitance were $10^{9} \mathrm{ohms}$ and $10 \mathrm{pf}$ respectively. The impedance matching was accomplished without any polarity change, and a high-meg resistor drained off any DC components such as those generated by temperature. This noninverting amplifier had a voltage gain of unity. Thus we needed additional signal-conditioning low noise amplifiers. These FET-MOS amplifiers were $6 \mathrm{~mm}$ in diameter. and $2.5 \mathrm{~cm}$ long. The sourcefollower amplifier installed in the instrument plug is shown in figure 6 .

The Princeton Applied Research low noise, lock-in amplifiers (PAR Model CR-4) powered by rechargeable nickel-cadmium battery packs* were selected. These amplifiers were provided with selectable panel switches for high and low frequency roll-off settings. In these tests the low frequency roll-off was set at $300 \mathrm{~Hz}$ and high frequency roll-off at $300 \mathrm{KHz}$. The schematic circuitry for the pressure sensors (Kulite and crystal) is given in figures $15(a)$ and (b). In order to obtain a low signal-to-noise ratio, the source impedance was adjusted by the addition of 2500 ohms in series with sensor impedance. Typical amplifier noise figure contours for the PAR CR-4 amplifiers are given in figure 16, and the selected operational range for the tests is shown in the hatched band in the figure.

In addition to these amplifiers several wide band differential DC amplifiers (Astrodata Model 886) were used for signal conditioning of sensors monitoring steady-state quantities like mean static pressure, surface temperatures, dynamic pressure, plate location in the test section, etc. The measured frequency response of the amplifiers (both PAR CR- 4 and Model 886) is given in figure 17 . The observed spread between amplifiers used in these tests fallswithin the band shown in figure 17. A mean curve is used for the data analysis. The PAR CR-4 amplifier data satisfy

\footnotetext{
* Battery operation is preferable to rectified 110 VAC power supply. There is always some residual ripple left over which cannot be eliminated and shows up as noise.
} 


$$
\text { Net Relative Gain } *=\frac{1}{\left[1+\left(f_{L F} / f\right)^{2}\right]\left[1+\left(f / f_{H F}\right)^{2}\right]}
$$

where $f, f_{L F}$ and $f_{H F}$ respectively denote the frequencies at which calibration data were taken, selected low frequency roll-off and the high frequency roll-off setting.

\section{Accelerometer}

An Endevco Accelerometer Model 2225 was mounted on the test model to monitor the mechanical vibrations during the pressure fluctuation measurements. A mounting stud (Endevco \#29863) was used to avoid electrical ground loop problems. The sensitivity of the accelerometer and amplifier combination $^{\star *}$ was adjusted to give $0.707 \mathrm{vrms} / 1 \mathrm{~g}$. The accelerometer traces for a few test runs are given in figure 18. They indicate that during initial insertion of the model into the test stream the acceleration is less than $1.5 \mathrm{~g}^{\prime} \mathrm{s}$ and after that time the shock mounting successfully isolates the model from the quick insert mechanism. A similar phenomenon occurs during the retraction phase.

\section{Temperature Sensor}

The thermocouple sensors used were manufactured by Heat Technology Laboratory (Model TCS-102, with chromel-constantan elements). The sensor is $0.8 \mathrm{~mm}$ in diameter and about $4 \mathrm{~cm}$ long and can be used in an environment where the steady-state operating temperature is $1250^{\circ} \mathrm{K}$. The time rise given by the manufacturer is around 1 usec.

The TCS Series thermocouple probes consists of a coaxial center lead wire (first thermocouple element) housed in a thick wall tube (second thermocouple element). The two metals are insulated from each other by a special aluminum oxide-type insulation which is effective to about

\footnotetext{
*Net Relative Gain = (amplifier output/amplifier input) $\times$ (1/gain setting) at the selected signal generator frequency.

** Source-follower amplifiers similar to the ones used for the crystal sensor were used with the accelerometer. In this case an Astrodata Amplifier 886 was used since it has an operational range from DC to $20 \mathrm{KHz}$.
} 
$1400^{\circ} \mathrm{K}$ for steady-state temperatures (and substantially higher for transient temperatures). The thermocouple junction is formed at the polished end of the coaxial assembly by a vacuum deposited metallic plating (see fig. 19). Lead wires were welded to the coaxial probe and embedded in a refractory insulating cement.

For measuring the local stream temperatures the probe was provided with a Kel-F (low thermal expansion and low coefficient of conductivity material) shielding. Without this shielding the thermocouple sensor would be in direct contact with the model and the effects of the model acting as a heat sink would be felt in the measurements.

\section{Correlator}

A Honeywell Model 9410-002 Time Delay Correlator was used in cbtaining auto- and cross-correlation functions from the recorded random pressure time histories of sensors A to $\mathrm{H}$. The Correlator was tested by using a sinusoidal signal up to $500 \mathrm{KHz}$ *

The Correlator accepts three input time histories simultaneously from the tape recorder, say $1,2,3$, and provides the auto-correlations $R_{11}(\tau)$, $R_{22}(\tau), R_{33}(\tau)$ and the cross-correlations $R_{12}(\tau), R_{23}(\tau), R_{31}(\tau)$. If the sensors are separated by fixed distances, the three space-time correlations are obtained directly when the output of the correlator is recorded, namely, $R_{12}\left(x_{1}, \tau\right), R_{23}\left(x_{2}, \tau\right)$ and $R_{31}\left(x_{3}, \tau\right)$.

The output of the correlator can be recorded with an XY plotter or as an oscilloscope trace captured on a polaroid. picture. Both the above methods were used for recording the analyzed data.

\section{Spectrum Analyzer}

For analyzing signals with random contents an estimate of the long term power spectrum (or power spectral density characteristics) is usually desired. For spectral analysis of the pressure data a Real Time Analyzer Model SD301B (Spectral Dynamics Corp.) was used. With its compatible Ensemble Averager Model SD302 an estimate of the average spectrum characteristics of a random signal can be performed up to a frequency of $20 \mathrm{KHz}$.

\footnotetext{
*Even though the manufacturers claim frequency response from $50 \mathrm{~Hz}$ to $250 \mathrm{KHz}$, the calibration tests carried out prior to our data analysis indicated this unit to be good far beyond $250 \mathrm{KHz}$.
} 
The confidence level of the input signal is improved directly in proportion to the number of ensemble averages that can be accomplished. With an SD302 unit the averaging is accomplished in the frequency domain by examining an ensemble of spectral functions, and then obtaining the average sum over a predetermined length of time.

The analysis frequency range of the combination of the SD301B and SD302 units can be extended up to 1 megahertz by adding a spectrum translator, SD303, and supplying an associated reference signal from a high quality Synthesizer (Hewlett Packard 3320A-002).

The power spectral plots were obtained with linear horizontal frequency scale and log vertical spectral density amplitude scale. The output is recorded on a polaroid picture from the oscilloscope display or used in an $X Y$ plotter.

\section{Root-Mean-Square Meter}

The root-mean-square values of the recorded pressure fluctuations were obtained for each of the eight pressure sensors with a Ballentine True RMS meter (Model 320A) which is good for frequencies from $5 \mathrm{~Hz}$ to $4 \mathrm{MHz}$, and has an accuracy of \pm 1 percent from the true RMS value.

\section{TEST PROCEDURE}

While the wind tunnel was being prepared for the test run with the preselected reservoir pressure and temperature conditions to obtain required test dynamic pressure, periodic calibrations of all the sensors were carried out using the portable shock tube apparatus, and the data were recorded on tape. Once this was performed, the side walls were closed and the model retracted into the quick insert chamber, in preparation for the actual tests. When the proper reservoir conditions were achieved, the hydraulically actuated valve at the pebble bed heater was opened. As soon as steady test section conditions were reached, the model was injected into the stream for a short duration. During the model's stay in the stream for one minute, the data were taken on the 20-channel tape recorder, both direct and FM modes of operation, at 3.048 meters per second. Immediately after the run the recorded data were visually examined on an oscilloscope in order to ascertain that no data were lost. 
At each wind-tunnel test condition four nearly identical tests were carried out in order to assure repeatability of tests and to increase the statistical confidence level of the recorded data during the analysis. .

At each test stream Mach number, visual flow observations were made to ascertain the location of the boundary-layer transition. The plate was sprayed with a saturated solution of Fluorene in a petroleum ether base before it was injected into the test section. Transition was found to occur 7 to $10 \mathrm{~cm}$ from the leading edge in all cases. This indicated that the inconel leading-edge portion and flat-plate surface did not match perfectly and thus acted as a triggering mechanism for the initiation of transition. This was an unexpected result, but assured a fully developed turbulent flow region over the instrument plug.

DATA ANALYSIS

The analysis of the recorded wall pressure data was first examined for the stationarity conditions. The pressures recorded by sensors $A$ to $H$ are the fluctuations about a mean static pressure; and the root-mean-square (RMS) of these fluctuations from any one of the sensors for a given test stream condition remained an invariant irrespective of the length of record examined (above an optimum minimum playback of about 5 seconds at a tape speed of $3.048 \mathrm{~m} / \mathrm{sec}$ ). The weak stationarity condition requires that first and second moments of time history of the pressure fluctuations be independent of time. These are satisfied by the results; namely, the time average of the fluctuating static pressure (about the mean wall static pressure) is zero and the RMS pressure is independent of time.

The instrument noise was recorded with the entire model in the test section, with no stream flow in the wind tunnel and with the outputs of all the sensors recorded at the same amplifier gains as those in the actual Mach 7.4 tests. The noise results given in terms of ratios of an equivalent RMS pressure for each sensor to dynamic pressure, $q_{\infty}$, are in figure 20. The curve corresponding to each sensor is labeled. 
MEAN AERODYNAMIC BOUNDARY-LAYER PARAMETERS

- The aerodynamic parameters necessary for reducing the measured pressure fluctuations to generally accepted nondimensional form are the boundarylayer thickness $(\delta)$, displacement thickness $(\delta *)$, momentum thickness $(\theta)$ and local skin-friction coefficient $\left(c_{f}\right)$. In the tests in-situ measurement of these parameters was not possible due to malfunctioning of the boundarylayer traverse apparatus. However, Bies (ref. 45) who examined the existing literature on turbulent boundary-layer flows on a flat-plate model, derived an empirical formula in terms of Reynolds number, $R_{x}$ and Mach Number, $M_{\infty}$. These empirical relations are given below:

$$
\begin{aligned}
& \frac{\delta}{\mathrm{x}}=0.37 \operatorname{Re}_{\mathrm{x}}^{-0.2}\left[1+\left(\frac{\operatorname{Re}_{\mathrm{x}}}{6.9 \times 10^{7}}\right)^{2}\right]^{-0.1} \\
& \frac{\delta^{*}}{\mathrm{x}}=\frac{\left(1.3+0.43 \mathrm{M}_{\infty}^{2}\right)(\delta / \mathrm{x})}{10.4+0.5 \mathrm{M}_{\infty}^{2}\left(1+2 \times 10^{-8} \mathrm{Re}_{\mathrm{x}}\right)^{0.333}} \\
& \frac{\delta^{*}}{\theta}=\left(1.3+0.43 \mathrm{M}_{\infty}^{2}\right)
\end{aligned}
$$

Using these empirical relationships and the procedure given by Bies for obtaining local skin-friction coefficient, $c_{f}$, the necessary boundary-layer parameters for present tasks were calculated from the known information on free-stream Mach number $M_{\infty}$ and the Reynolds number at the center of the instrument plug, $R e_{X}$ and these are given in Table $I$. These results were compared with some available data on a similar flat-plate model tested in the Ames Research Center 3.5-Foot Hypersonic wind Tunnel (ref. 46), and the agreement is good. In all the above considerations one has to keep in mind that our model is essentially a cold plate (i.e., surface temperature less than adiabatic wall temperature) in the test stream.

\section{RESULTS}

$\tilde{\mathrm{p}}$ Variation with $\mathrm{q}_{\infty}$

The measured RMS surface pressures, $\tilde{p}$, from each sensor $A$ to $H$ are plotted against dynamic pressure, $q_{\infty}$ and are given in figures $21(a),(b)$ 
(c) and (d) for the three Mach numbers; namely, 5.2, 7.4, and 10.4. Within the expected experimental scatter the RMS pressures obtained from the Kulite or the crystal sensor, located at the same downstream position from the leading edge, were found to be equal; that is, $\tilde{\mathrm{p}}_{\mathrm{A}} \doteq \tilde{\mathrm{p}}_{\mathrm{E}}, \tilde{\mathrm{p}}_{\mathrm{B}}=\tilde{\mathrm{p}}_{\mathrm{F}}$ and so on. This indicates that the overall energy contribution from the high frequency portion of the power spectral density plots towards RMS pressure value is small. Hence, in all the discussions on the overall statistical properties of the pressures no distinction will be made regarding the two types of sensors. In figure 21 the RMS pressures are plotted against dynamic pressure, $q_{\infty}$ and show that the pressures increase with the dynamic pressure, $\mathrm{q}_{\infty}$ and seem to reach a maximum around $q_{\infty}=5 \times 10^{4} \mathrm{~N} / \mathrm{m}^{2}$. A composite plot of all the RMS pressures shown in figure 22 is obtained from. figure $21(a)$ to (d), in order to extract the general trend from the data. The RMS pressures from sensor A to $D$ or from sensor $\mathrm{E}$ to $\mathrm{H}$ satisfies

$$
\tilde{\mathrm{p}}_{\mathrm{A}, \mathrm{E}}: \tilde{\mathrm{p}}_{\mathrm{B}, \mathrm{F}}: \tilde{\mathrm{p}}_{\mathrm{C}, \mathrm{G}}: \tilde{\mathrm{p}}_{\mathrm{D}, \mathrm{H}}:=1: 0.96: 0.92: 0.89
$$

and these are expressible in terms of the percent change of Reynolds number from sensor to sensor as compared with that of the leading sensor; namely, A or E. That is,

$$
\tilde{p}_{i}=\tilde{p}_{A, E} \exp (300 R)
$$

where

$$
R=\left(\frac{R e_{A}-R e_{i}}{R e_{A}}\right) \frac{1}{100}
$$

$i$ assumes A, B, C or D according to the sensor in consideration. Further, as Mach number increases, the value of $\tilde{p}$ decreases at any given $q_{\infty}$. The orientation of sensors with respect to mean stream direction is given in figure 5. In figure 22 the solid lines, obtained by the method of least squares, represent the measured data. An analytic expression has been obtained which fits all the measured data and is given below. 


$$
\begin{aligned}
\tilde{\mathrm{p}}_{\mathrm{A}, \mathrm{E}}= & {\left[1.1 \times 10^{-2} \mathrm{q}_{\infty}-6.959 \times 10^{-8} \mathrm{q}_{\infty}^{2}-4.138\right] \mathrm{exp}-\left[\left(1.017 \times 10^{-1}\right.\right.} \\
& \left.\left.+.1 .305 \times 10^{-6} \mathrm{q}_{\infty}-1.261 \times 10^{-11} \mathrm{q}_{\infty}^{2}\right) \mathrm{M}_{\infty}\right]
\end{aligned}
$$

This expression is valid for $5.2<M_{\infty}<10.4$ and for $6.5 \times 10^{3} \leq q_{\infty} \leq$ $7 \times 10^{4} \mathrm{~N} / \mathrm{m}^{2}$. The dashed lines were obtained from calculation of the analytic expression given in equation (7) for the sensor $A$ or $E$. For convenience the ordinate scale at the right in figure 22 is given in $\mathrm{db}^{\prime} \mathrm{s}$ based on reference RMS pressure equal to $2 \times 10^{-5} \mathrm{~N} / \mathrm{m}^{2}$.

\section{$\tilde{\mathrm{p}} / \mathrm{p}$ Variation with $\mathrm{q}_{\infty}$}

In figure 23(a), (b), (c) and (d) the ratio of the RMS pressure to the mean static pressure at the surface, $\tilde{\mathrm{p}} / \mathrm{p}$ is plotted against dynamic pressure, $q_{\infty}$ for sensors $A$ to $H$. The ratio of $\tilde{p} / p$ decreases as $q_{\infty}$ is increased from $2 \times 10^{4} \mathrm{~N} / \mathrm{m}^{2}$ for $M_{\infty}=5.2$ and 7.4 tests. But, for Mach number 10.4 , this ratio of $\tilde{p} / p$, within the available data, increases as $\mathrm{q}_{\infty}$ is increased up to $2 \times 10^{4} \mathrm{~N} / \mathrm{m}^{2}$. A composite plot obtained by separating the data for each Mach number 5.2, 7.4 and 10.4 is given in figure 24(a), (b) and (c) respectively. The composite plot (fig. 24) showsithat $\tilde{p} / p$ reaches a maximum value around $q_{\infty}=2 \times 10^{4} \mathrm{~N} / \mathrm{m}^{2}$ for $M_{\infty}=7.4$ and then rapidly falls off to a small value as $q_{\infty}$ is increased. Wind-tunnel operational limitations severely hampered efforts to obtain data for $\mathrm{q}_{\infty}<2 \times 10^{4} \mathrm{~N} / \mathrm{m}^{2}$ at $\mathrm{M}_{\infty}=5.2$ or 7.4 ; and for $\mathrm{q}_{\infty}>2 \times 10^{4} \mathrm{~N} / \mathrm{m}^{2}$ at $M_{\infty}=10.4$. Thus, the entire range of $q_{\infty}$, for a given Mach number, was not possible. All the same it is of interest here to point out that the RMS pressures vary from a maximum of 25 percent to a minimum of 5 percent of the mean static pressure and depend on the dynamic pressure, $q_{\infty}$. From the experimental results of Kistler and Chen (ref. 22), one notes the value of $\tilde{p}$ to vary from 8 to 10 percent of the mean static pressures at $M_{\infty}=5$, while chen (ref. 21) reports for the same test conditions a vari-. ation of up to 20 percent. Laufer (refs. 19 and 20) while examining the far field problem observed $\tilde{\mathrm{p}} / \mathrm{p}$ equal to 1 percent outside the turbulent boundary layer, and pointed out the need for more experimental information in the near field at $M_{\infty}>5$. Thus the present data seem to be more in agreement with Chen's data than with Kistler and Chen's results. 


$$
\tilde{\mathrm{p}} / \mathrm{q}_{\infty} \text { Variation with } \mathrm{q}_{\infty} \text { and } \mathrm{M}_{\infty}
$$

The ratios of the RMS pressure, $\tilde{p}$ to the free-stream dynamic pressure, $\mathrm{q}_{\infty}$ are given in figures $25(\mathrm{a}),(\mathrm{b}),(\mathrm{c})$ and (d) for each sensor and for all the three tested Mach numbers. These values are considerably smaller than the $6 \times 10^{-3}$ value obtained for the subsonic case (refs. 9 and 10). Further, the present data differ from the subsonic results in that the ratio $\tilde{p} / q_{\infty}$ depends on $q_{\infty}$. Kistler and Chen (ref. 22) obtained $\tilde{p} / q_{\infty}$ around $5 \times 10^{-3}$ at $M_{\infty}=5$ and the dependence of this ratio on $q_{\infty}$ was not evident from their data. In figure 26 we have reproduced the exact plots given in Chen's thesis (ref. 21, fig. 16). He gives $\tilde{p} / q_{\infty}$ variation as a function of $R e_{\delta *}$ and $M_{\infty} ;$ and this is an order of magnitude larger than the data obtained from present tests (see fig. 28).

The composite plot of $\tilde{\mathrm{p}} / \mathrm{q}_{\infty}$ against $\mathrm{q}_{\infty}$ in figure 27 is obtained from replotting data from figure 25 from all the sensors. It is interesting to compare the data with that of figure 20, the instrument noise plot for each sensor and the associated electronics for $M_{\infty}=7.4$ tests.

In order to assess the signal to noise in the instrumentation, we obtain the ratio of $\tilde{\mathrm{p}} / \mathrm{q}_{\infty}$ from figure 27 and divide it by the converted noise value (from fig. 20 in equivalent $\tilde{p} / q_{\infty}$ terms) at the selected $q_{\infty}$ and $M_{\infty}$. As an example consider sensor $A$, at $M_{\infty}=7.4$ and $q_{\infty}=5 \times 10^{4} \mathrm{~N} / \mathrm{m}^{2}$. In this case we obtain a signal to noise ratio of $2.5 \times 10^{-3} / 1.7 \times 10^{-4}=14.7$. Thus, the signal is nearly 15 times larger than the threshhold noise from all the electronic amplifiers in circuit for sensor $A$ at $q_{\infty}=5 \times 10^{4}$ and $M_{\infty}=7.4$.

In figure $28, \tilde{\mathrm{p}} / \mathrm{q}_{\infty}$ is replotted against the free-stream Mach number, $M_{\infty}$ for sensor $A$ or $E$. The data shown inside the boxes are obtained from figure $25(\mathrm{a})$. It can be seen as $q_{\infty}$ increases the $\tilde{p} / q_{\infty}$ ratio tends towards Lowson's empirical curve (ref. 27). The data obtained in these tests generally fall between Houbolt (ref. 48) and Lowson's empirical curves and are smaller than the value obtained at subsonic speeds. * The data in the range of free-stream Mach number, $8.25<M_{\infty}<9$ are from Harvey et al. (ref. 37) who measured the velocity and temperature fluctuations through the use of electron-beam techniques. From these

ॠ The vertical scale in these plots is logarithmic and does exaggerate the spread of data from the empirical curves. 
measurements they were able to calculate the pressure fluctuations indirectly. These are shown in figure 28 and at high $\mathrm{q}_{\infty}$ the data from the present investigation tends toward their results. In addition Harvey et al. calculated $\tilde{p} / q_{\infty}$ values using Wallace's data for adiabatic and cold wall conditions and these are in figure 28 at $M_{\infty}=9$. The adiabatic wall data of Harvey's coincides with that of Lowson's (ref. 47). The results from Chen (ref. 2l) are an order of magnitude above all of other known experimental results.

Variation of $\tilde{\mathrm{p}} / \mathrm{q}_{\infty}$ as a Function of $R e_{\delta *}$ and $\tilde{\mathrm{p}} / \tau_{\mathrm{w}}$ as a Function of $\operatorname{Re}_{\theta}$ In figure $29, \tilde{\mathrm{p}} / q_{\infty}$ is plotted against the Reynolds number based on displacement thickness, $\mathrm{Re}_{\delta^{*}}$. The data satisfies the relation

$$
\frac{\tilde{p}}{q_{\infty}} \times 10^{3}=\left(40.587-1.678 M_{\infty}\right) R_{\delta *}^{-0.205}
$$

The variation of $\tilde{\mathrm{p}} / \mathrm{q}_{\infty}$ as a function of $\operatorname{Re}_{\delta}$ suggests that one should examine these RMS pressures using local skin friction or local wall shear stress, $\tau_{w}$ ' as the characteristic nondimensionalizing parameter. Hence, we have plotted the RMS pressure data in terms of $\tilde{p} / \tau_{w}$ against the Reynolds number based on momentum thickness, $\mathrm{Re}_{\theta}$. The data shown in figure 30 may be represented by the relation

$$
\frac{\tilde{\mathbf{p}}}{\tau_{\mathrm{W}}}=\mathrm{A}_{2} \operatorname{Re}_{\theta}^{\mathrm{B}}
$$

where $A_{2}=0.28$ and $B_{2}=0.27$.

The $\tilde{\mathrm{p}} / \tau_{\mathrm{w}}$ data obtained in the present experiments for a given $\operatorname{Re}_{\theta}\left(=4.3 \times 10^{4}\right)$ is presented in terms of $M_{\infty}$ in figure 31. The data in general are lower in value than Kistler and Chen (ref. 22). Also, in figure 32. we have reproduced data from Chen's thesis (ref. 21). These are considerably higher than any other results. In general the present data show the same trends as speaker and Ailman (ref. 24) even though they are slightly higher in value. In the present investigation no special efforts were made to hold $\operatorname{Re}_{\theta}$ constant in order to obtain $\tilde{p} / \tau_{w}$ shown in figure 31. But fortunately the test range covered a sufficient number of $\operatorname{Re}_{\theta}$ values from which the data for $\operatorname{Re}_{\theta}=4.3 \times 10^{4}$ could be selected for all the three Mach numbers considered. 
In figure 31 the data from the present investigation are extended to the subsonic range. This extrapolated value is in agreement with the results of Williams (ref. 31) and Belcher (ref. 32).

\section{Spectral Considerations}

The power spectrum of the recorded time history of the pressure fluctuations from each sensor $A$ to $H$ was carried out using a real time spectral analyzer and the associated electronic units described in the section on instrumentation. The entire spectral analyzer, a composite of several compatible electronic units, was calibrated by checking for the line spectral output for sinusoidal inputs of known RMS voltage in the frequency range from $\mathrm{I} \mathrm{KHz}$ to $\mathrm{I} \mathrm{MHz}$. The performance was also checked by examining the output of the analyzer for a square wave input at $1 \mathrm{KHz}$ and $20 \mathrm{KHz}$ and comparing the spectrum with the theoretical results. In addition, a random white noise generator of known RMS voltage with a frequency range up to $20 \mathrm{KHz}$ was used as an input signal, and the spectral output obtained was compared with the known results.

The nondimensional pressure spectral density and frequency were chosen to be of the form $\mathrm{p}^{2}(\mathrm{f}) \mathrm{U}_{\infty} / \mathrm{q}_{\infty}^{2} \delta *$ and $\mathrm{f} \delta * / \mathrm{U}_{\infty}$ respectively. The choice of these parameters was due to the ready availability of the aerodynamic parameters $\mathrm{U}_{\infty}$ and $q_{\infty}$. Also, the physically meaningful boundary-layer thickness, $\delta *$ is retained. Each of the spectral curves represent 256 ensemble averages.

The nondimensional pressure spectral density plots are given in figures 33, 34 and 35 for the three Mach numbers investigated. The plots represent a composite of spectral data obtained by analyzing the data three times in frequency range of (i) $1 \mathrm{KHz} \leq \mathrm{f} \leq 20 \mathrm{KHz}$, (ii) $3.2 \mathrm{KHz} \leq \mathrm{f} \leq$ $160 \mathrm{KHz}$, and (iii) $25 \mathrm{KHz} \leq f \leq 320 \mathrm{KHz}$. In each of these plots there are eight curves, each representing the output of one particular sensor, $A$ to $H$ as indicated. On the first line at the top of each plot the test run number and the RMS pressures of sensors $A$ to $H$ (PRMS, $N / \mathrm{m}^{2}$ ) are given. The second line contains pertinent test condition information; namely, the free-stream dynamic pressure, $\mathrm{q}_{\infty}\left(\mathrm{QINF}, \mathrm{N} / \mathrm{m}^{2}\right)$, the stream velocity, $\mathrm{U}_{\infty}$ (UINF, $\mathrm{m} / \mathrm{sec}$ ), Reynolds number in millions, $\mathrm{Re}_{\mathrm{x}}$ (Reynolds number), and the displacement thickness $\delta^{*}$ (DELSTR, $m$ ). Using the Reynolds number $\operatorname{Re}_{x}$ given in the plots and the aerodynamic parameters $\delta, \delta *, \theta$ and $c_{f}$ given in Table $I$, any of the commonly accepted nondimensionalizing parameters can be obtained for replotting the presented data. 
The RMS pressures in figures 33-35 were obtained using Simpson's rule for area integration of the spectral density plot and were checked against the values that were directly obtained through the use of a Ballantine meter. The agreement between these two ways of obtaining $\tilde{p}$ is within 2 percent of the reduced pressure data.

One common noticeable feature of the spectral density plots is that a sharp drop occurs at large strouhal number, $f \delta^{*} / \mathrm{U}_{\infty}$. This characteristic drop is attributable to the finite size of the sensor. As the turbulent eddy size becomes comparable or smaller than the sensor diameter, the sensor response falls off drastically. In a convected turbulent boundary layer flow the small eddies correspond to high frequency components. Thus, at some high frequency (determined by the ratio of sensor diameter to boundary layer displacement thickness, $d / \delta^{*}$ ) cancellation between positive and negative pressure fluctuations begin to occur over the sensor surface. willmarth (ref. 12), Serafini (ref. 18) and others have suggested on the basis of their experimental investigations that the ratio of $d / \delta$ * is to be kept as small as possible in order to obtain high frequency (small eddy size) resolution of the convected pressure field. In figure 36 the effect of the ratio of sensor diameter $d$, to displacement thickness, $\delta *$ is indicated. In addition, as the size of the sensor is reduced the sensitivity is decreased. In spite of all these considerations, the finite physical size of the sensor allows us to record only the pressure that has suffered some attenuation through the filtering effect of the measuring instrument. In order to obtain the true value from the measured quantity a correction is essential if $d / \delta^{*}$ is large. Corcos (ref. 49), Willmarth and Roos (ref. 14), Chandiramani (ref. 50), Gilchrist et al. (ref. 51) and White (ref. 52) investigated this problem both experimentally and theoretically. From their results one is able to obtain the expected reduction in pressure sensitivity of the sensor due to its finite size; and this is given in figure 37. In the present investigation using an average free-stream velocity of $1.4 \mathrm{Km} / \mathrm{sec}$ the sensors satisfy the following condition.

$$
\begin{aligned}
& \text { Kulite Sensor : } 0.10<\frac{\mathrm{d}}{\delta^{\star}}<0.35 ; \text { and } 0.3<\frac{\omega \mathrm{d}}{\mathrm{U}_{\infty}}<0.65 \\
& \text { Crystal sensor: } 0.03<\frac{\mathrm{d}}{\delta^{\star}}<0.10 ; \text { and } 0.3<\frac{\omega_{\mathrm{d}}}{\mathrm{U}_{\infty}}<0.75
\end{aligned}
$$


From figure 12, the calculated attenuation for the crystal sensor used in the tests at $300 \mathrm{KHz}$ is equal to $0.3 \mathrm{dbs}$. Thus, the corrections for the size of the sensor are negligible and hence no corrections were made.

For a free-stream Mach number of 5.2, the power spectral density exhibits some scatter at low strouhal number between adjacent measuring stations. Similar results have been observed by previous investigators while carrying out tests in the supersonic region of $M_{\infty}=3.5$ (refs. 24 and 27). However, at higher strouhal numbers all these data coalesce nicely. Various explanations have been offered for low strouhal number behavior; namely, some weak shock wave interaction, random eruptions of turbulence bursts due to surface roughness and the probability of having a region where the boundary layer turbulence is not fully developed. In the present tests one finds that the pebbles in the heater chamber are iiterally lifted up and settled back for the high dynamic pressure and mass flows at a test section Mach number of 5.2. This process releases all the disintegrated alumina oxide particles as dust. In order to alleviate the problem a periodic wind-tunnel dust blow-down, without the model in the stream was carried out. In spite of this precaution the problem of dust was present for the Mach 5.2 tests.

The phenomenon of dust in the stream disappears at Mach numbers of 7.4 and 10.4 and is one of the unexplained idiosyncrasies of the tunnel operation. The effect on the power spectral density plot is less scatter of data at these Mach numbers $\left(M_{\infty}=7.4\right.$ or 10.4$)$.

In order to obtain the general trends and to arrive at an analytic expression, a composite of several of these power spectral density plots for a given Mach number were examined. The spectral density is in general satisfied by the following equation for all the Mach numbers considered.

$$
G(f)=\frac{p^{2}(f) U_{\infty}}{q_{\infty}^{2} \delta^{*}}=\frac{A^{2}}{\left[1+\left(f \delta * / U_{\infty}\right)^{2}\right]^{3 / 2}}
$$

The constant A is related to the RMS pressure as can be seen by simple integration.

$$
\frac{\overline{p^{2}}(f) U_{\infty}}{q_{\infty}^{2} \delta^{*}}=\frac{U_{\infty}}{\delta^{*}} \int_{0}^{\infty} \frac{A^{2} d\left(f \delta * / U_{\infty}\right)}{\left[1+\left(f \delta^{*} / U_{\infty}\right)^{2}\right]^{3 / 2}}
$$


i.e.,

$$
\frac{\tilde{\mathrm{p}}}{\mathrm{q}_{\infty}}=\sqrt{\frac{\overline{\mathrm{p}^{2}}(f)}{\mathrm{q}_{\infty}^{2}}}=\left\{\left[\frac{\mathrm{A}^{2}\left(\mathrm{f} \delta * / \mathrm{U}_{\infty}\right)}{\sqrt{1+\left(\mathrm{f} \delta * / \mathrm{U}_{\infty}\right)^{2}}}\right]_{0}^{\infty}\right\}^{1 / 2}
$$

i.e.,

$$
\frac{\tilde{p}}{q_{\infty}}=A
$$

An analytic expression for $\tilde{p} / q_{\infty}$ which fits most of the measurements has already been obtained (eq. (7)). Thus, all the power spectral density plots for the pressure fluctuations obtained in these tests can be collapsed by considering

$$
\frac{p^{2}(f) U_{\infty}}{q_{\infty}^{2} \delta^{*}} \cdot \frac{1}{A^{2}}=\frac{1}{\left[1+\left(f \delta^{*} / U_{\infty}\right)^{2}\right]^{3 / 2}}
$$

i.e.,

$$
\frac{p^{2}(f) / q_{\infty}^{2}}{\left(\tilde{p} / q_{\infty}\right)^{2}} \cdot \frac{U_{\infty}}{\delta^{*}}=\frac{1}{\left[1+\left(f \delta^{*} / U_{\infty}\right)^{2}\right]^{3 / 2}}
$$

Lowson (ref. 47) suggested a similar expression after examining some of the supersonic experimental results. The amplitudes of the power spectral density from the plots presented are in general lower than the measurements given by Chen (ref. 21) Kistler and Chen (ref. 22), Coe (refs. 27 and 28) and others. The data presented by Speaker and Ailman (ref. 24) for attached turbulent boundary layer flow agrees with results of this investigation. This can be predicted from $\tilde{p} / q_{\infty}$ plots versus Mach numbers (see fig. 32) which indicates lower $\tilde{p} / q_{\infty}$ values as $M_{\infty}$ is increased for a given $q_{\infty}$ value.

\section{Correlation Functions}

The correlation functions of interest in the present work are the space-time and auto correlations of the recorded surface pressure fluctuations. The space-time correlation is defined as 


$$
R_{i j}\left(\vec{x}, \vec{\xi}_{i} \tau\right)=\frac{\overline{p_{i}(\vec{x} ; \tau) p_{j}(\vec{x}+\vec{\xi} ; t+\tau)}}{\tilde{p}_{i} \tilde{p}_{j}}=R_{i j}(\xi, \tau)
$$

where the correlation function is normalized by the use of appropriate RMS pressures. Two-point space-time correlations provide information on the convective velocity of the pressure fluctuations and on the decay rate of the pressure signature as it is convected downstream.

The auto-correlation function is a special case of the space-time correlation and is obtained by letting the space separation vector $\vec{\xi}$ between sensors go to zero. This essentially implies that the pressure fluctuation considered is the output of one sensor with the usual time displacements carried out for obtaining the correlation function. In this case, since $\vec{\xi}=0, i=j$. For a stationary process the auto correlation can also be obtained by considering the Fourier transform of the power spectral density function.*. This can be expressed by using equation (10) and considering its Fourier transform. Hence

$$
R(\tau)=\frac{1}{\tilde{p}^{2}}\left(q_{\infty}^{2} \frac{\delta^{*}}{U_{\infty}}\right) \int_{0}^{\infty} S(f) e^{i 2 \pi f t} d f=\frac{1}{\tilde{p}^{2}} q_{\infty}^{2} A^{2} \cdot \int_{0}^{\infty} \frac{e^{i B f^{*}} d f *}{\left(1+f^{*}\right)^{3 / 2}}
$$

where

$$
E^{*}=\frac{f \delta^{*}}{U_{\infty}}, B=2 \pi \frac{U_{\infty} t}{\delta^{\star}} \text { and } q_{\infty} A=\tilde{p}
$$

and finally

$$
R(\tau)=\int_{0}^{\infty} \frac{e^{i B E^{*}}}{\left(1+f *^{2}\right)^{3 / 2}} d f^{*}
$$

This integration can be carried out by contour integration methods.

The entire correlation analysis has been carried out for the freestream Mach number of 7.4 using the correlator described in the section

\footnotetext{
* The pressure fluctuation data satisfy the stationarity condition; and thus the auto correlation $R_{i j}(\tau)$ and the power spectral density function, $\mathrm{p}^{2}(\mathrm{f})$ are Fourier transforms of each other.
} 
on instrumentation. This analysis is not as exhaustive as the considerations of RMS pressures and the power spectral densities. In addition, in this analysis only broad frequency bands were considered ( $1 \mathrm{KHz} \leq \mathrm{f}$ $\leq 300 \mathrm{kHz})$. The pressure sensors are located a fixed distance apart $\xi_{0}$ in the stream direction in order. to measure the longitudinal statistical characteristics of pressure fluctuations. No lateral correlation consideration was undertaken in this investigation.

Two space-time correlations can be analyzed at one time with the correlator, which accepts three separate pressure data as input simultaneously and yields output voltages proportional to the correlation function, for instance, $\mathrm{R}_{\mathrm{AB}}\left(\xi_{0}, \tau\right)$ and $\mathrm{R}_{\mathrm{BC}}\left(\xi_{0}, \tau\right)$ or $\mathrm{R}_{\mathrm{AC}}\left(2 \xi_{0}, \tau\right)$. In order to obtain all the three space-time correlations of interest with this correlator the analysis had to be performed twice for each set of test conditions. Care was exercised to examine the data in the same time interval for both the correlation analyses. Thus the correlations $R_{A B}\left(\xi_{O}, \tau\right)$ and $R_{A C}\left(\xi_{O}, \tau\right)$ from analyzing the data the first time, and $R_{A C}\left(\xi_{O}, \tau\right)$ and $\mathrm{R}_{\mathrm{AD}}\left(\xi_{O}, \mathrm{~T}\right)$ from the second analysis were obtained. In figure 38 only one trace of the common $\mathrm{R}_{A C}\left(\xi_{O}, \tau\right)$ correlation is gj.ven.

The convective velocity can be defined in two ways, each giving a slightly different result. The two convective velocities are defined as

$$
\mathrm{U}_{\mathrm{C}_{1}}=\frac{\left|\xi_{\mathrm{O}}\right|}{\tau}
$$

where: $\tau$ corresponds to a $\tau$ that satisfies the condition

$$
\frac{\partial R(\xi, \tau)}{\partial \tau}=0
$$

and

$$
U_{\mathrm{C}_{2}}=\frac{\left|\xi_{0}\right|}{\tau}
$$

where $\tau$ corresponds to a $\tau$ that satisfies the condition

$$
\frac{\partial R(\xi, \tau)}{\partial|\xi|}=0
$$


From the present analysis only $U_{C_{1}}$ can be obtained since the sensors fixed in the instrument plug cannot be easily varied in their separation distances from each other. Thus one measures the time delay, $\tau$, required between the peak values of two consecutive.correlations, for instance $R_{A B}$ and $\mathrm{R}_{A C}$, or $\mathrm{R}_{A C}$ and $\mathrm{R}_{A D}$, and since the displacement distance between. sensors are known we obtain the so-called convective velocity as equal to $\xi / \tau$..

The convective velocities so obtained are in the range

$$
0.60 \leq \frac{U_{C_{1}}}{U_{\infty}}<0.70
$$

and these values agree reasonably well with measurements given by Kistler and Chen (ref. 22), Chyu and Hanly (for $\xi_{1} / \delta^{*}=1.5-2.0$, ref. 53) and Bull (ref. 15). Even though a systematic space-time correlation for the other two Mach numbers $M_{\infty}=5.2$ and 10.4 were not performed, the results indicate Kistler and Chen's (ref. 22) findings of the $U_{C} / U_{\infty}$ ratio as a: function of $M_{\infty}$ to be valid; namely, $U_{C} / U_{\infty}$ decreases from: a value of 0.8 at subsonic flow to 0.65 at supersonic and remains constant beyond $M_{\infty}=3.5$. Similar results have been obtained by coeri(ref. 27). From än. . examination of shadowgraph pictures Jedlick et al. (ref. 54). reported convective velncities of. bursts of turbulence in the boundary layer to be about 60 percent of the free-stream velocity.

From the space-time correlation considerations $\mathrm{U}_{\mathrm{C}} / \mathrm{U}_{\infty}=0.65$ was obtained for a separation distance of $\xi_{\mathrm{O}}=0.95 \mathrm{~cm}$, and $\mathrm{U}_{\mathrm{C}} / \mathrm{U}_{\infty}=0.700$ for a separation distance of $2 \xi_{0}=1.9 \mathrm{~cm}$. Thus, as the separation distance increases, there is a siight increase in $U_{c} / U_{\infty}$. This is in agreement with the work of Bull (ref. 15) who reports an increase in the $\mathrm{U}_{\mathrm{C}_{1}} / \mathrm{U}_{\infty}$ value as separation distance increases for the subsonic case.

These results indicate that the small scale (or large wave number) components of pressure fluctuations travel downstream slowly and lose their identity rapidly; while the large scale (or small wave number) components are convected rapidly and lose their identity slowly.

The auto-correlation plot.is useful if one is interested in the microscale of pressure fluctuations (analogous to a velocity field). A representative auto-correlation plot is given in figure 39. The vertical scale in these plots are relative and are the voltage outputs of the correlator. An osculating parabola is drawn in dashed lines in order to obtain the microscale length of the pressure fluctuating field. The average of the 
intercept of this curve on the abscissa is around $50 \mu \mathrm{sec}$. If one uses the average convective velocity of $900 \mathrm{~m} / \mathrm{sec}$ then the microscale is equal to $50 \times 10^{-6} \times 900 \times 100=4.5 \mathrm{~cm}$, and is greater than the distance between sensors.

\section{CONCLUSIONS}

Fluctuating pressures have been measured on a flat-plate model at hypersonic Mach numbers of $5.2,7.4$ and 10.4 beneath an attached turbulent boundary layer for a range of Reynolds numbers from 1 to $15 \mathrm{millions}$ and for a dynamic pressure range of 6800 to $66000 \mathrm{~N} / \mathrm{m}^{2}$. The following conclusions can be drawn from the analysis.

1. With proper care the piezoelectric transducers could be successfully used in a pebble heated hypersonic wind tunnel, for measuring surface pressure fluctuations.

2. Over the range of Mach number tested, the PMS pressures increase with the dynamic pressure and reach a plateau. For any dynamic pressure, the RMS pressure decreases with an increase in Mach number. The pressure intensity decreases systematically as the pressure signature is convected downstream from sensor to sensor.

3. The variation of the RMS pressures normalized with mean static pressure is 8 to 25 percent, and the peak values occur at.dynamic pressures around $2 \times 10^{4} \mathrm{~N} / \mathrm{m}^{2}$.

4. The ratios of RMS pressures to dynamic pressure all fall below $4 \times 10^{-3}$ for these hypersonic wind-tunnel tests and this ratio decreases as dynamic pressure or Mach number is increased. For high dynamic pressure level at hypersonic speeds, the normalized RMS pressure to dynamic pressure could be approximated by Lowson's empirical formula (ref. 27).

5. The ratio of RMS pressure to dynamic pressure varies as $\left(\operatorname{Re}_{\delta^{*}}^{-0}{ }^{2}\right)$ for all three test Mach numbers.

6. The ratio of RMS pressure to wall shear stress falls between 2 and 3 and considerably lower than Kistler and Chen's results (ref. 22). As Mach number increases, this ratio decreases in accordance with the trends observed by Speaker and Ailman (ref. 24). 
7. The nondimensionalized power spectral density $p^{2}(f) U_{\infty} / q^{2} \delta$ plots against frequency parameter $f \delta * / U_{\infty}$ show that all the plots can be collapsed to a single analytic representation given in equation (15). The contribution from high frequency components (100-300 $\mathrm{kHz}$ ) of the pressure fluctuations to the pressure intensity $(\tilde{p})$ is very small. Even though the amplitudes of the high frequency components are small, these might be of interest for structural response considerations leading to fatigue problems.

8. The space-time correlation considerations yield convective velocities that are equal to 0.7 of free-stream velocities.

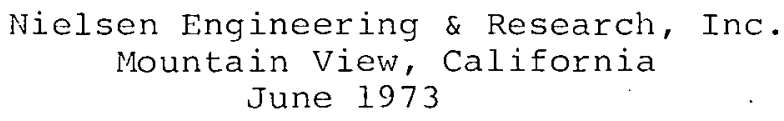


REFERENCES

1. Phillips, O. M.: Surface Noise from a Plane Turbulent Boundary Layer. British Aeronautical Research Council 16, 963-FM 2099, Aug. 1954.

2. Phillips, O. M.: On the Aerodynamic Surface Noise from a Plane Turbulent Boundary Layer. Proc. Royal Soc., Series A, vol. 234, pp. 327335, Feb. 1956.

3. Curle, N.: The Influence of Solid Boundaries Upon Aerodynamic Sound. Proc. Royal Society, Series A, vol. 231, pp. 505-513, Sept. 1955.

4. Kraichnan, Robert H.: Pressure Field within Homogeneous Anisotropic Turbulence. Jour. Acoustical Soc. Am., vol. 28, pp. 64-72, Jan. 1956.

5. Kraichnan, Robert H.: Pressure Fluctuations in Turbulent Flow over a Flat. Plate. Jour. Acoustical Soc. Am., vol. 28, pp. 378-390, May 1956.

6. Kraichnan, Robert H.: Noise Transmission from Boundary Layer Pressure Fluctuations. Jour. Acoustical Soc. Am., vol. 29, no. 1, Jan. 1957.

7. Lilley, G. M. and Hodgson, T. H.: On Surface Pressure Fluctuations in Turbulent Boundary Layers. Note 101, The College of Aero.

(Cranfield), 1960. (See also Revised Rep. 276, AGARD, Apr. 1960)

8. Harrison, M.: Pressure Fluctuations on the Wall Adjacent to a Turbulent Boundary Layer. David Taylor Model Basin Rept. 1260, Dec. 1958.

9. Willmarth, W. W.: Wall-Pressure Fluctuations in a Turbulent Boundary Layer. Jour. Acoustical Soc. Am., vol. 28, pp. 1048-1053, Nov. 1956.

10. Willmarth, W. W.: wall Pressure Fluctuations in a Turbulent Boundary Layer.. NACA TN 4139 , Mar. 1958.

11. Willmarth, W. W.: Space-Time Correlations and Spectra in a Turbulent Boundary Layer. Jour. of the Aeronautical Sciences, vol. 25, May 1958.

12. Willmarth, W. W.: Space-Time Correlations and Spectra of Wall Pressure in a Turbulent Boundary Layer. NASA Memo 3-17-59W, Mar. 1959.

13. Willmarth, W. W. and Wooldridge, C. E.: Measurements of the Fluctuating Pressure at the Wall beneath a Thick Turbulent Boundary Layer. Univ. of Mich. Tech. Rept. 02920-1-T, Apr. 1962. Also Jour. Fluid Mech., vol. 14, p. 187, 1962 .

14.. Willmarth, W. W. and Roos, F. W.: Resolution and structure of the Wall Pressure Field beneath a Turbulent Boundary Layer. Jour. of Fluid Mech., vol. 22, part 1, pp. 81-94, May 1965.

15. Bull, M. K. and Willis, J. L.: Some Results of. Experimental Investigations of the Surface Pressure Field due to a Turbulent Boundary Layer. ASD Tech. Documentary Rept. 62-425, Aug. 1962. Also A.A.S.U. Rept. 199, Nov. 1961. 
16. Bull, M. K., Wilby, J. F., and Blackman, D. R.: Wall Pressure Fluctuations in Boundary Layer Flow and Response of Simple structures to Random Pressure Fields. Univ. of Southampton, A.A.S.U. Rept. 243, July 1963.

17. Bull, M. K.: Wall-Pressure Fluctuations Associated with Subsonic Turbulent Boundary. Layer Flow. Jour. of Fluid Mech., vol. 2-, pt. 4, p. 719,1967 .

18. Serafini, J. S.: Wall-Pressure Fluctuations and Pressure-Velocity, Correlations in a Turbulent Boundary Layer. NASA'TR R-165, Dëc: 1963. Also AGARD Rept. 453, 1963.

19. Laufer, John: Sound Radiation from a Turbulent Boundary Layer. Proceedings of the Marseille Conf. on Turbulence; Aug. 1961. Published as CNRS Rept. 108, Paris, 1962.

20. Laufer, John: Some Statistical Properties of the Pressure Field Radiated by a Turbulent Boundary Layer. Physics of Fluids, vol. 7 , no. 8, Aug. 1964 .

21. Chen, W. S.: Measurements of Aerodynamic ivoise on a.Flat Plate in Supersonic Flow. Aero. Engr. Thesis, Cal. Inst. of Tech., 1961.

22. Kistler, A. L. and Chen, W. S.: The Fluctuating Pressure Field in a Supersonic Turbulent Boundary Layer. JPL Tech. Rept. no. 32-277, Aug. 1962. Also Jour. Fluid Mech., vol. 16, p. 41, 1963.

23. Lilley, G. M.: Wall Pressure Fluctuations under Turbulent Boundary Layers at Subsonic and Supersonic Speeds. Cranfield College of Aeronautics Note 140, Mar'. 1963. Also AGARD Rept. 454, Apr. 1963.

24. Speaker, W. F. and Ailman, C. M.: Spectra and Space-Time Correlations of the Fluctuating Pressures at a Wall beneath a Supersonic Turbulent Boundary Layer Perturbed by Steps and Shock Waves. Douglas Rep. SM-49806, Nov. 1965.

25. Murphy, J. S., Bies, D. A., Speaker, W: V., and Franken, P. A.: Wind Tunnel Investigation of Turbulent Boundary Layer Noise as Related to Design Criteria for High Performance Vehicles. NASA TN D-2247, Apr. 1964.

26. Maestrello, L.: Use of Turbulent Model to Calculate the Vibration and Radiation Responses of a Panel, with Practical Suggestions for Reducing Sound Level. Jour. of Sound \& Vib., vol. 5, pp. 407-448, 1967 .

27. Coe, C. F. and Chyu, W. J.: Pressure-Fluctuation Inputs and Response of Panels underlying Attached and Separated Supersonic Turbulent Boundary Layers. NASA TM X-62,189, Sept. 1972.

28. Coe, C. F, and Rechtien, R. D.: Scaling and spatial Correlation of Surface Pressure Fluctuations in Separated Flow at Supersonic Mach Numbers. Presented at AIAA Structural Dynamics \& Aeroelasticity Specialists Conference, New Orleans, LA, Apr. 16-17, 1969. 
29. Hodgson, T. H.: Pressure Fluctuations in Shear Flow Turbulence. Cranfield College of Aeronautics Note 129, 1962.

30. Mull, H. R. and Algranti, J. S.: Flight Measurements of Wall Pressure Fluctuations and Boundary Layer Turbulence. NASA TN D-280, Oct. 1960.

31. Williams, D. J. M.: Measurements of the Surface Pressure Fluctuations in a Turbulent Boundary Layer in Air at Supersonic Speeds. Univ. of Southampton, A.A.S.U. Rept. 162, Dec. 1960.

32. Belcher, P. M.: Predictions of Boundary-Layer Turbulence Spectra and Correlations for Supersonic Flight. Presented at the 5 th Intern. Acoustical Congress, Liege, Belgium, Sept. 1965.

33. Bhat, W. V.: Flight Test Measurement of Exterior Turbulent Boundary Layer Pressure Fluctuations on Boeing Model 737 Airplane. Jour. of Sound \& Vib., vol. 14, pp. 439-457, Feb. 1971 .

34. Lewis, T. L. and McLeod, N. J.: Flight Measurements of Boundary Layer Noise on the $\mathrm{X}-15$. NASA TN D-3364, March 1966.

35. Lewis, T. L., Jules, B. Dods, Jr., and Hanly, R. D.: Measurements of Surface Pressure Fluctuations on the XB-70 Airplane at Local Mach Numbers up to 2.45. NASA TN D-7226, Mar. 1973.

36. Wallace, J. E.: Hypersonic Turbulent Boundary Layer Measurements using an Electron Beam. Cornell Aero. Labs Rept. No. AN-2112-Y-1, Aug. 1968 .

37. Harvey, W. D., Bushnel1, D. M., and Beckwith, I. E.: Fluctuating Properties of Turbulent Boundary Layers for Mach Numbers up to.9. NASA TN D-5496, Oct. 1969.

38. Iberall, A. S.: Attenuation of Oscillatory Pressures in Instrument Lines. Jour. of Res., Nat. Bur. of Standards, vol. 45, pp. 85-108, July 1960 .

39. Chubb, T. W.: The Response of a Narrow Bore Pressure Measuring System to Step and Oscillatory Pressures. Royal Aircraft Estab. Tech. Rept. 68010 , Jan. 1968.

40. Ames Research Staff: Equations, Tables, and Charts for Compressible Flow. NACA Rept. 1135, 1953.

41. Raman, K. R.: Subminiature Transducer, Measures Unsteady Pressures. NASA Tech. Brief 71-10114, May 1971.

42. Blitz, T.: Fundamentals of Ultrasonics. London, Butterworth, 1963.

43. Kendall, D. N.: Dynamic Calibration of Pressure Transducers. Eng. Labs, McDonnell Aircraft Co.; presented at the 33rd Semi-Annual Meeting of the Supersonic Tunnel Assoc., El Segundo, CA, May 7-8, 1970 . 
44. Lederer, P. S. and Smith, P. O.: An Experimental Technique for Determination of the Fidelity of the Dynamic Responses of Pressure Transducers. Nat. Bur. of standards Rept. 7862, May 1963.

45. Bies, D. A.: A Review of Flight and Wind Tunnel Measurements of Boundary Layer Pressure Fluctuations and Induced Structural Response. NASA CR $626,1966$.

46. Hopkins, E. J., Keener, E. R., and Polek, T. E.: Hypersonic Turbulent Skin-Friction and Boundary-Layer Profiles on Nonadiabatic Flat Plates. AIAA vol. 10, no. 1, pp. 40-48, Jan. 1972 .

47. Lowson, M. V.: Prediction of Boundary Layer Pressure Fluctuations. AFFDL-TR-67-167, 1967.

48. Houbolt, J. C.: On the Estimation of Pressure Fluctuations ir. Boundary Layers and Wakes. Gen. Elec. Tech. Inf. Services Rept. 66 SD 296, Aug. 1966.

49. Corcos, G. M.: Resolutions of Pressure in Turbulence. Jour. Acoust. Soc. Am., vol. 35, no. 2, pp. 192-199, Feb. 1963.

50. Chandiramani, K. L.: Interpretation of Wall Pressure Measurements Under a Turbulent Boundary Layer. Bolt, Beranek and Newman Inc., Rep. No. 1310, Aug. 1965:

51. Gilchrist, R. B. and Strawderman, W. A.: Experimental Hydrophone-Size Correction Factor for Boundary-Layer Pressure Fluctuations. Jour. Acoust, Soc. Am., vol. 38, no. 2, pp. 298-302, Aug. 1965.

52. White, P. H.: Effects of Transducer Size, Shape, and Surface Sensitivity on the Measurement of Boundary Layer Pressures. Jour. Acoust. Soc. Am., vol. 41, no. 5, 1967.

53. Chyu, W. J. and Hanly, R. D.: Power and Cross-Spectra and Space-Time Correlations of Surface Fluctuating Pressures at Mach Numbers Between 1.6 and 2.5. NASA TN D-5440, sept. 1969.

54. Jedlicka, J. R., Wilkins, Max E. and Seiff, A.: Experimental Determinations of Boundary-Layer Transition on a Body of Revolution at $M=3.5$. NACA TN 3342, Oct. 1954. 


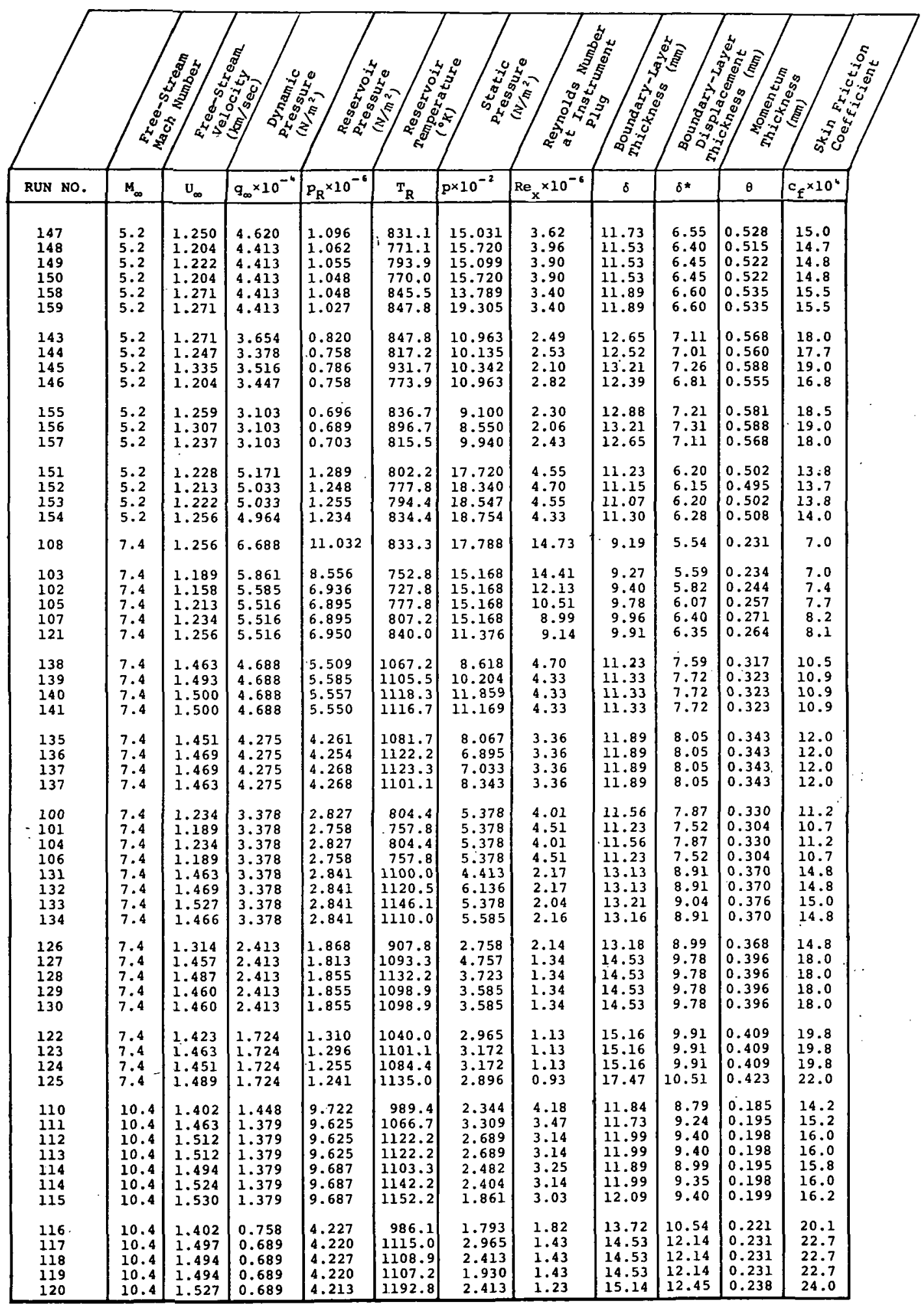

Table 1.- Summary of test conditions and aerodynomic porometers. 


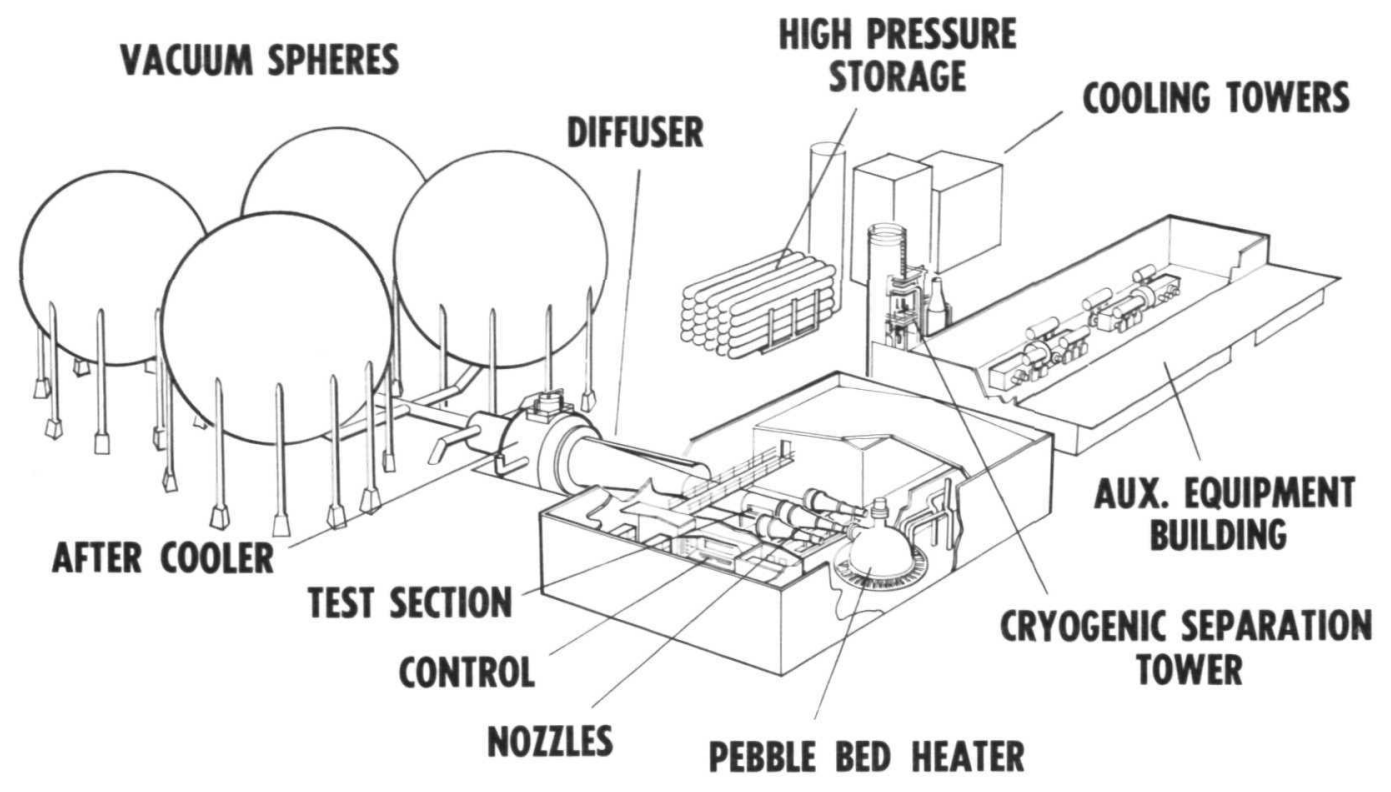

(a) Overall view of the test facility

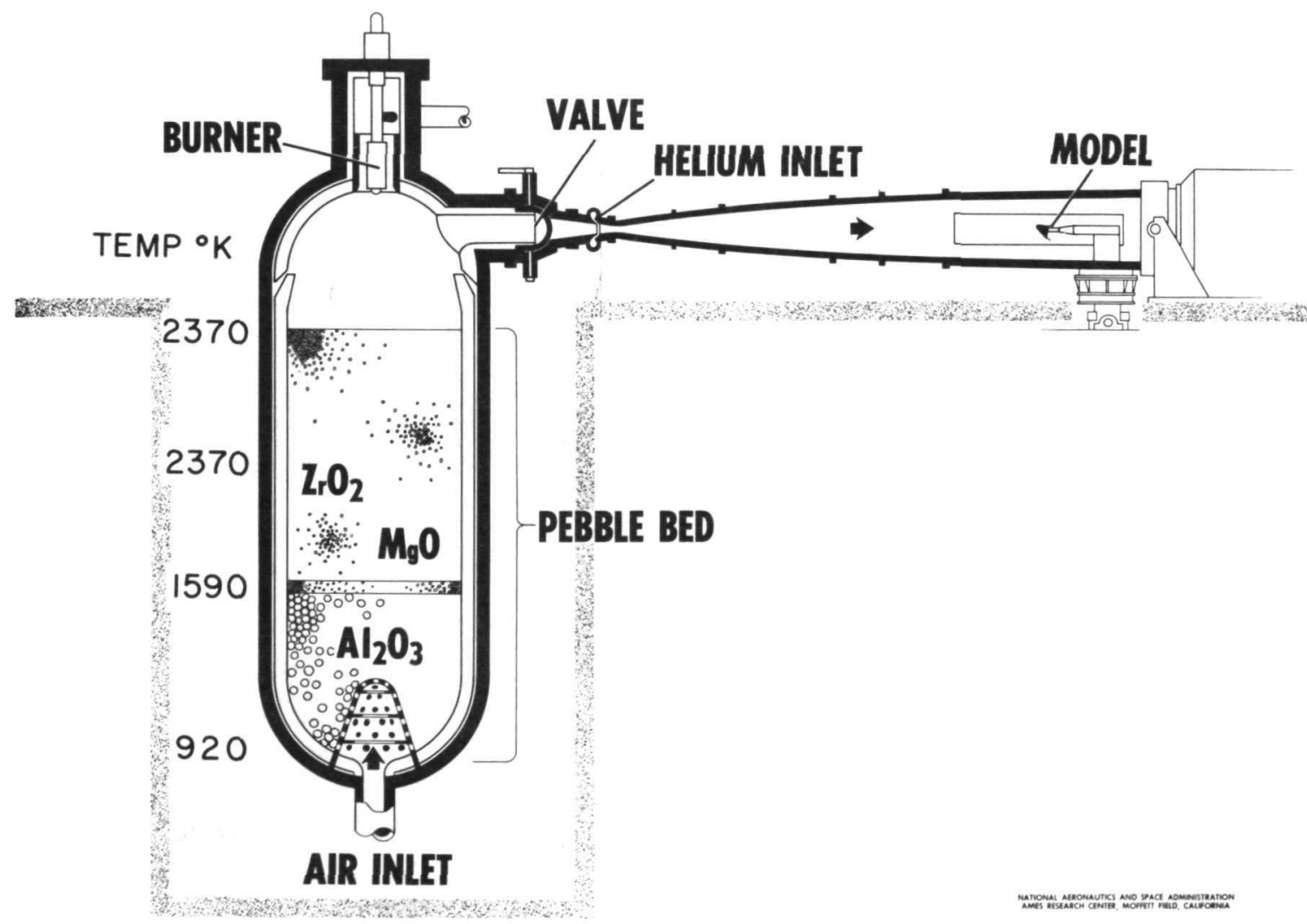

(b) Pebble bed heater, nozzle and test section

Figure 1.-Ames 3.5-Foot Hypersonic Wind Tunnel. 


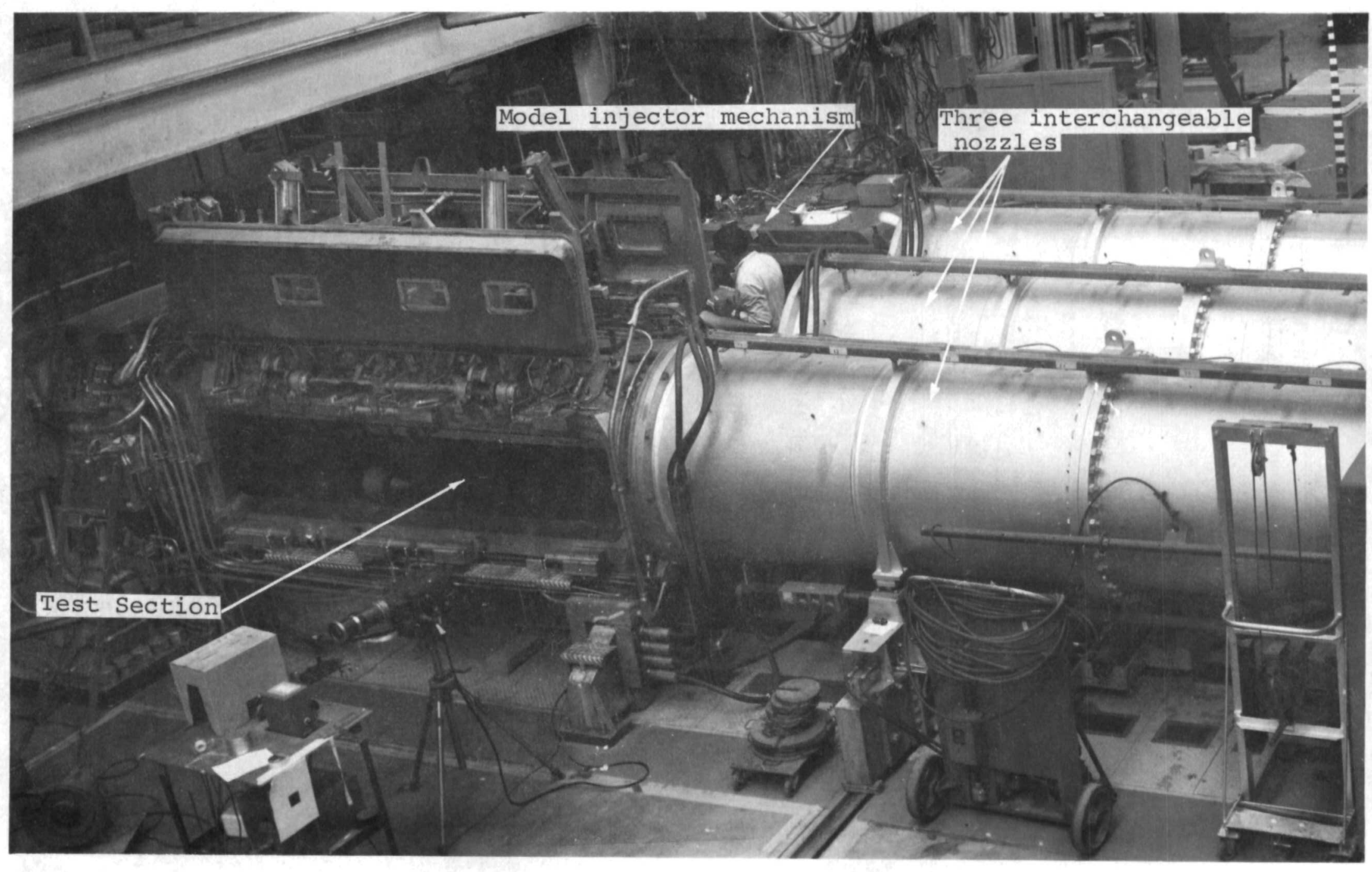

Figure 2.- Model injector, test section, and interchangeable nozzles. 


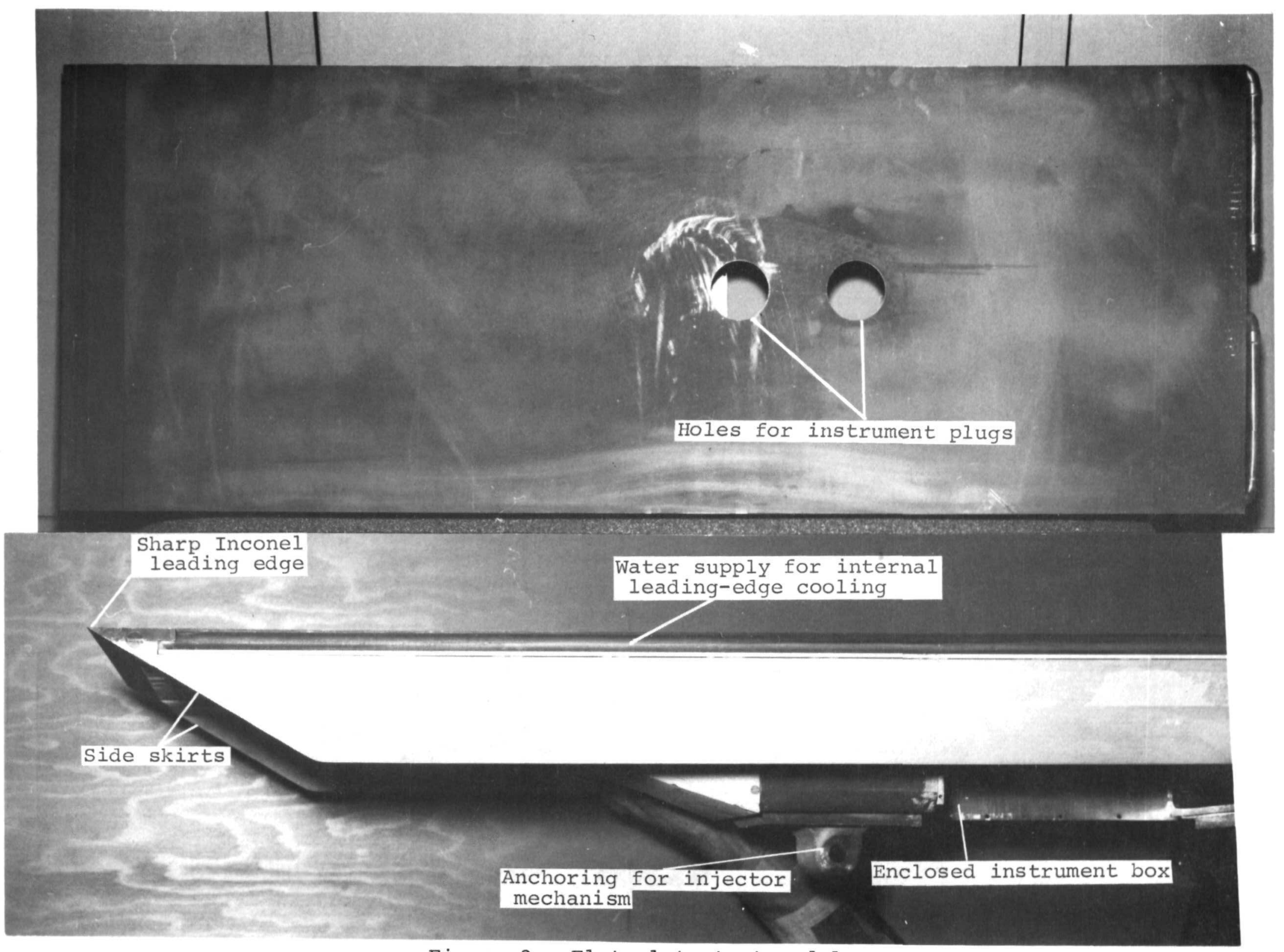

Figure 3.- Flat-plate test model. 


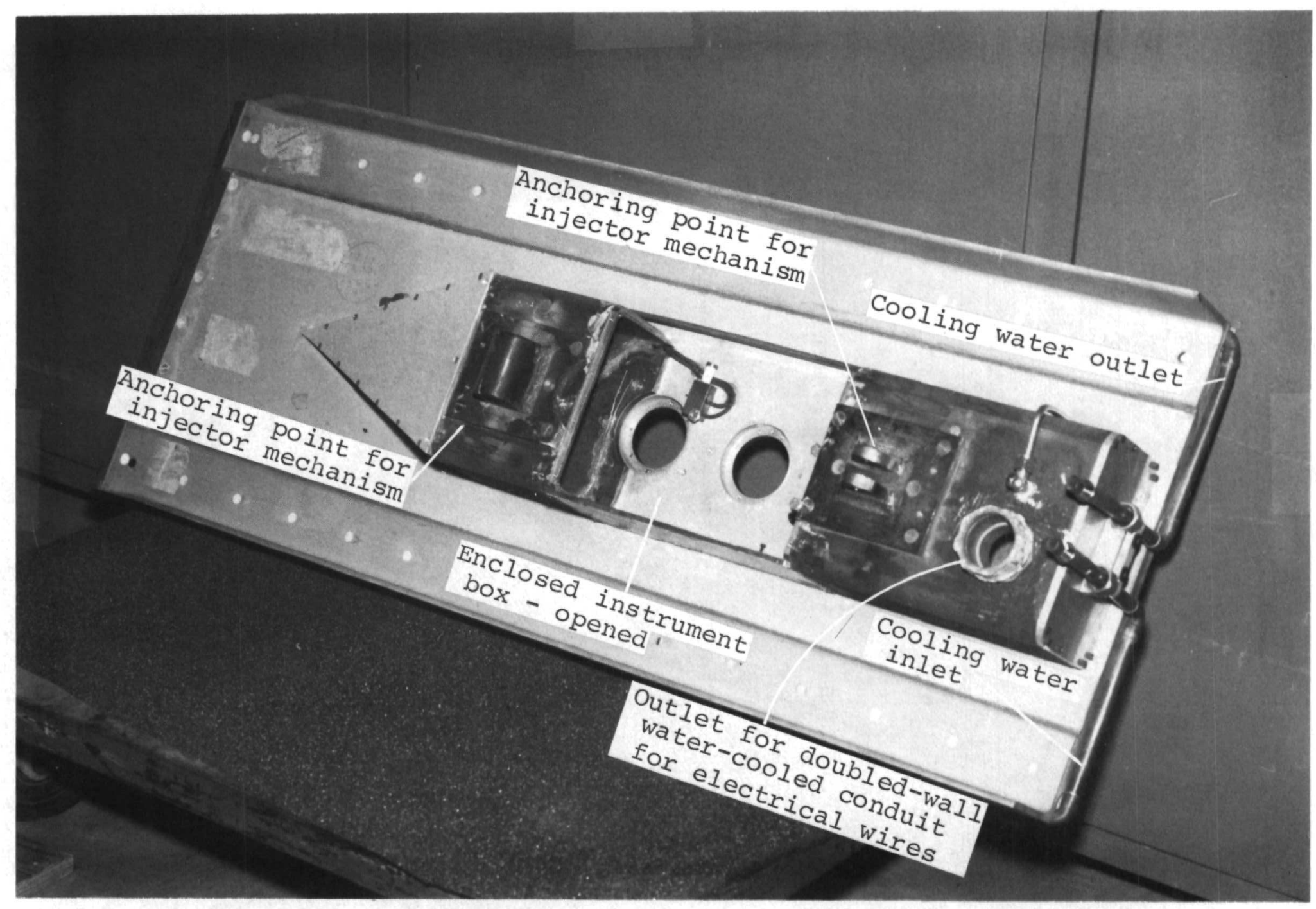

Figure 4.- Test model and protective enclosure for the instruments. 


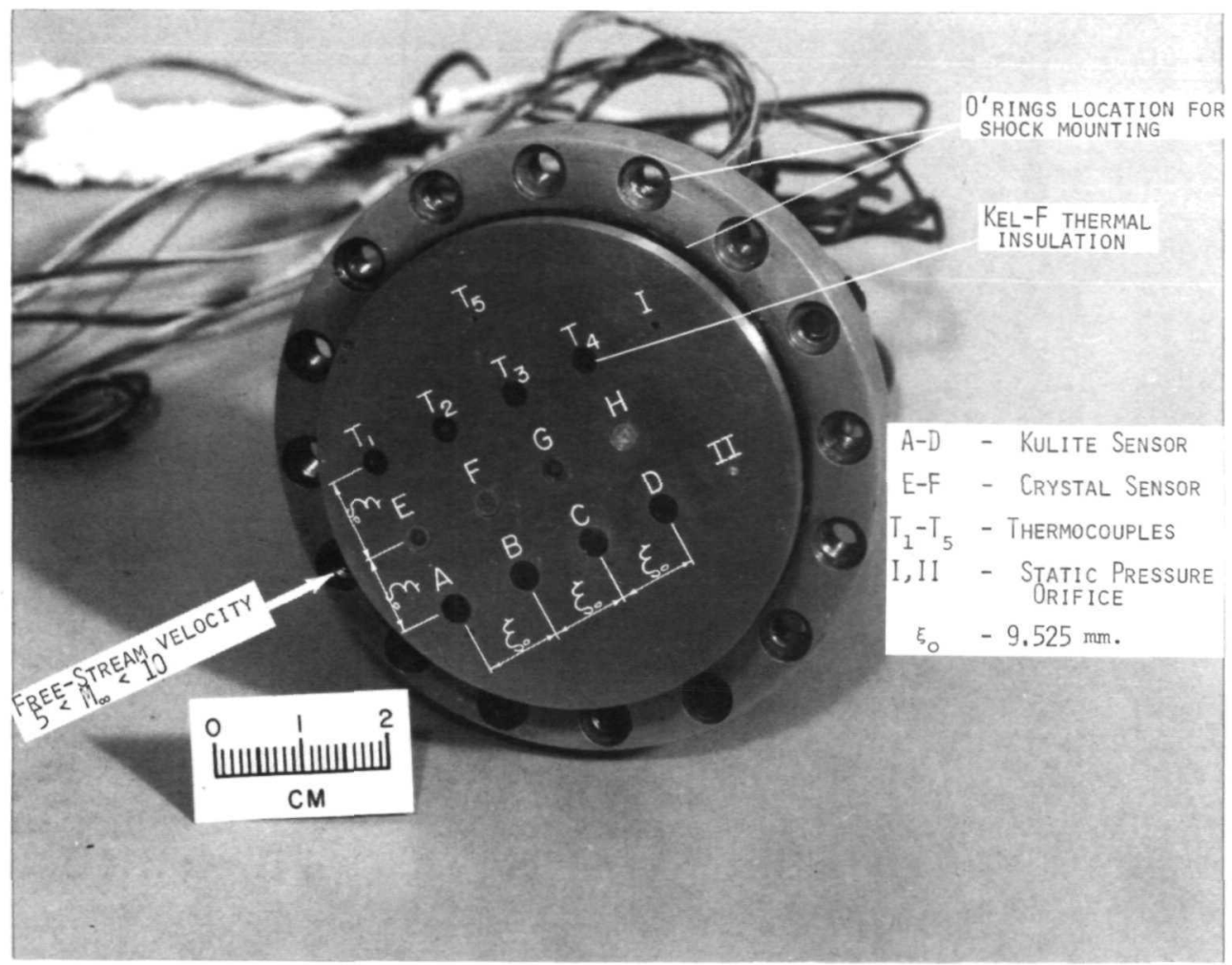

Figure 5.- Instrument plug showing various sensors

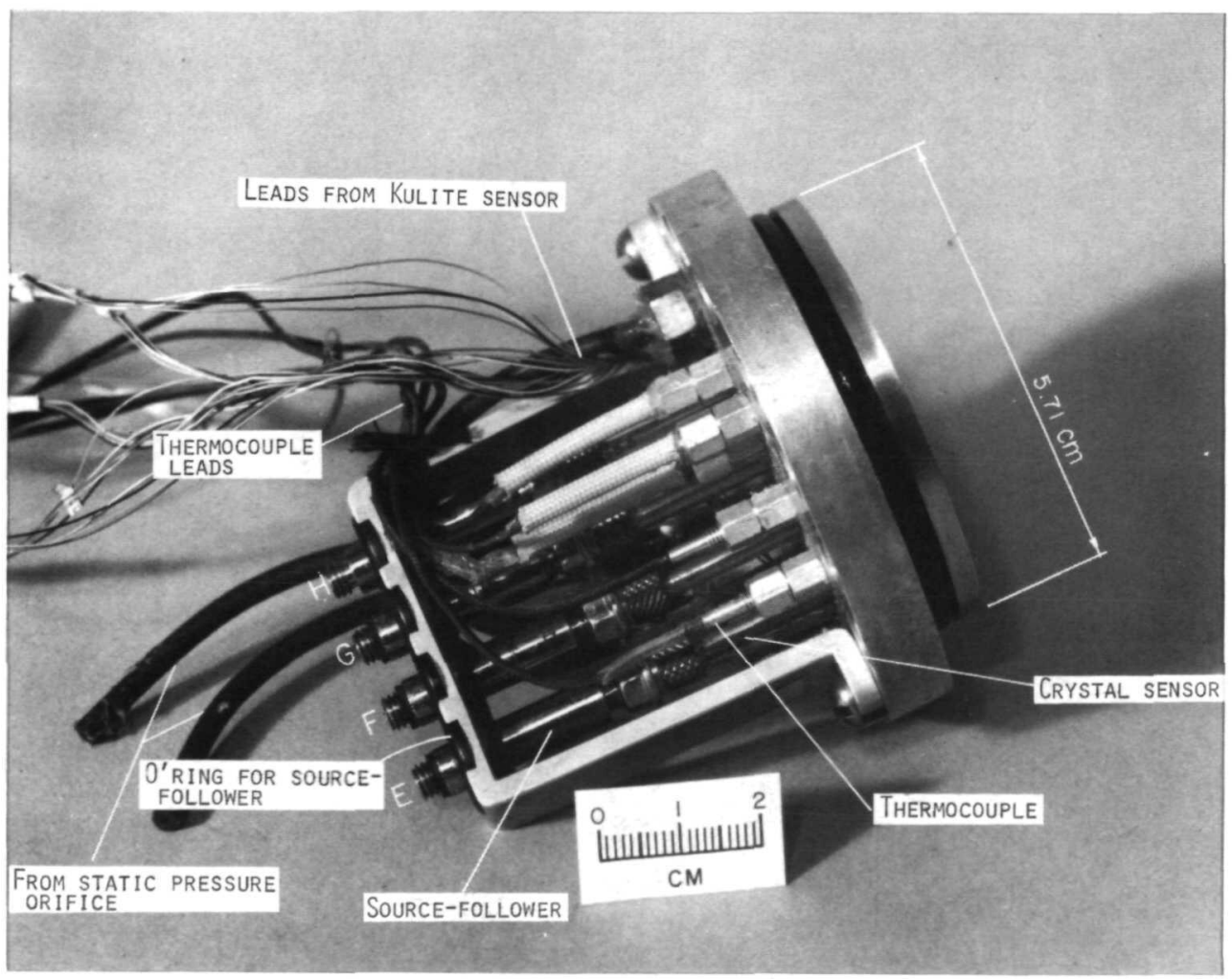

Figure 6.- Underside of the instrument plug showing sensors and signal conditioning amplifiers. 


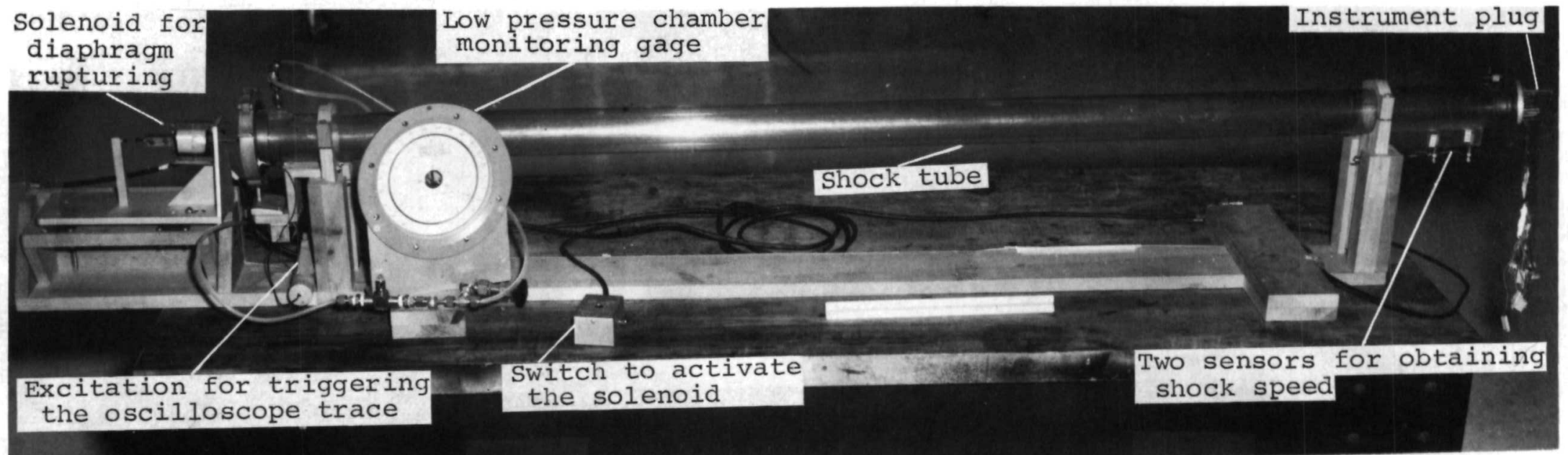

Figure 7.- Shock-tube calibration apparatus. 


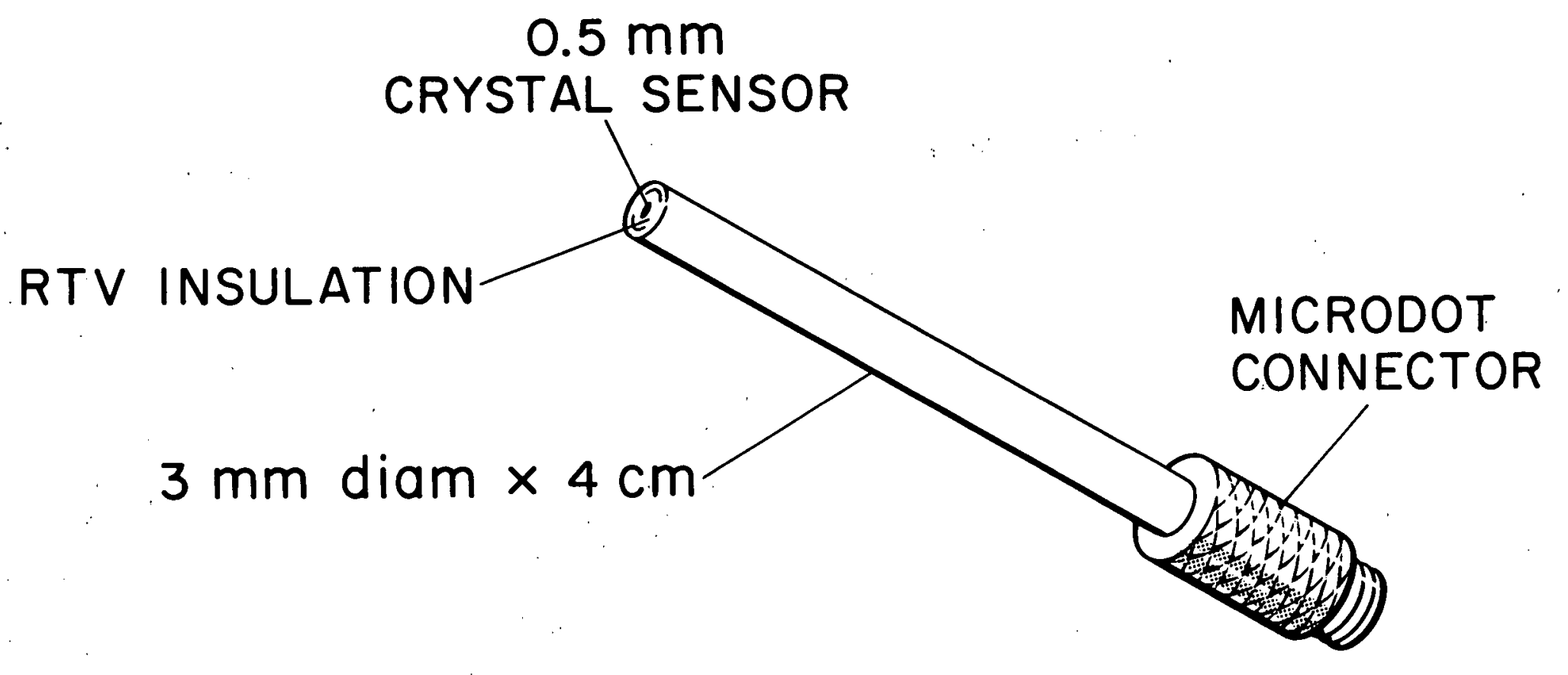

Figure 8.- Schematic diagram of crystal, sensor. 


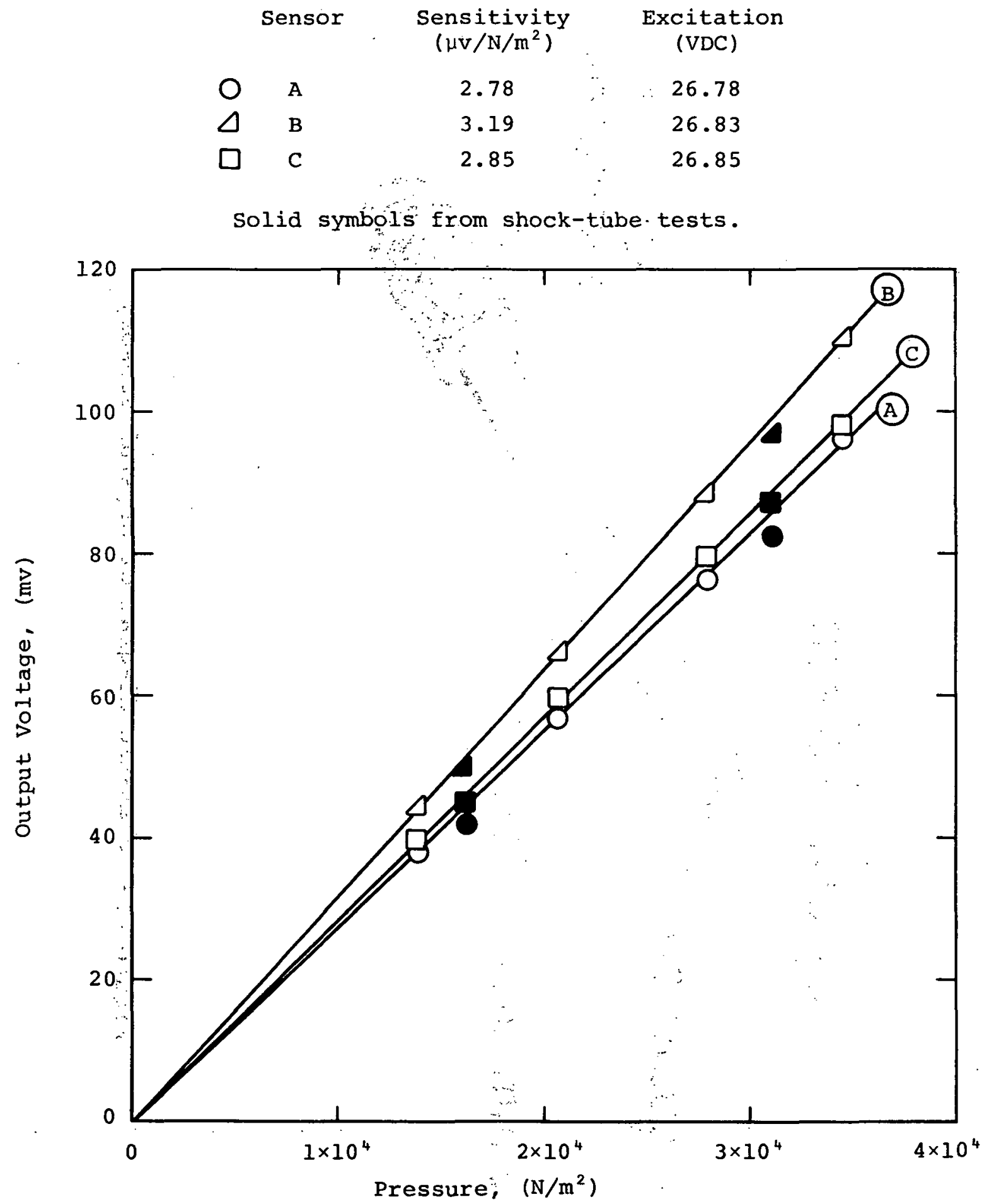

(a) Static and dynamic calibration data Kulite sensors $A, B$ and $C$.

Figure 9.- Calibration curves for pressure sensors. 


$\begin{array}{cccc}\text { Sensor } & \begin{array}{c}\text { Sensitivity } \\ \left(\mu \mathrm{v} / \mathrm{N} / \mathrm{m}^{2}\right)\end{array} & \begin{array}{c}\text { Excitation } \\ (\mathrm{VDC})\end{array} \\ \triangle & \text { D } & 2.95 & 26.84 \\ \nabla & \text { I } & 2.88 & 26.74 \\ \square & \text { II } & 3.30 & \therefore 2.85\end{array}$

Solid symbols from shock-tube tests.

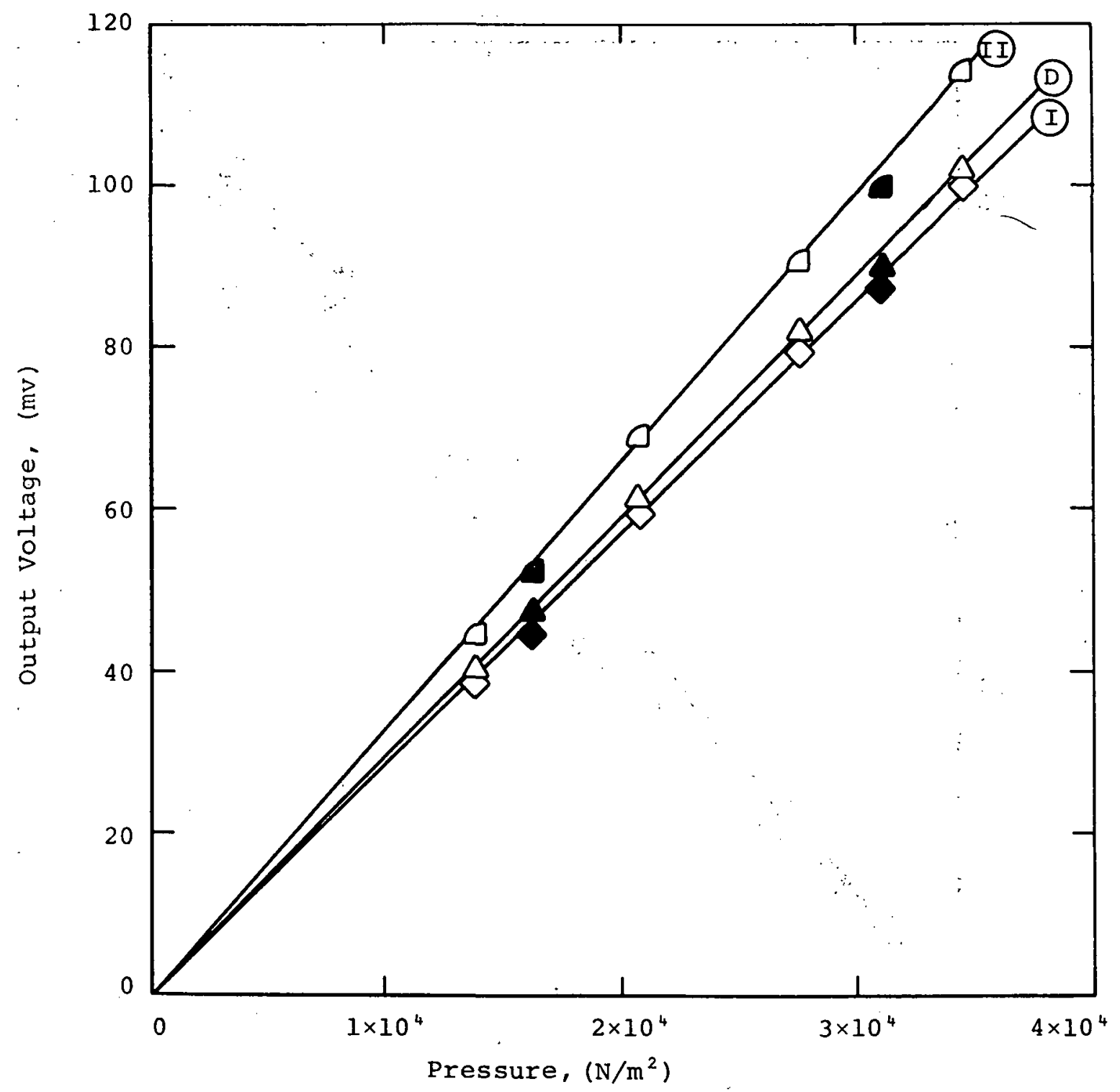

(b) Static and dynamic calibration data Kulite sensors $D, I$ and II.

Figure $9 .-$ Continued. 


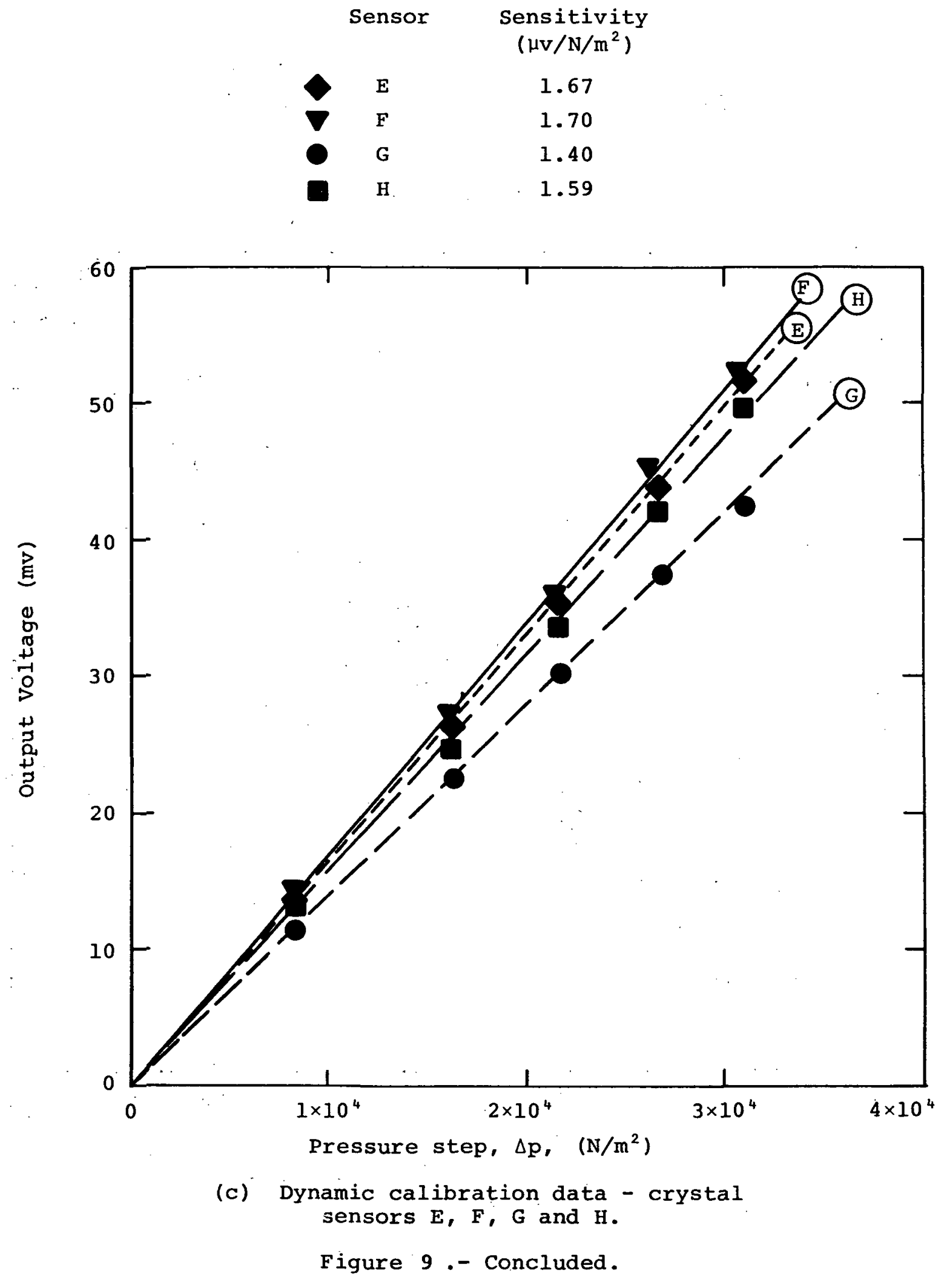




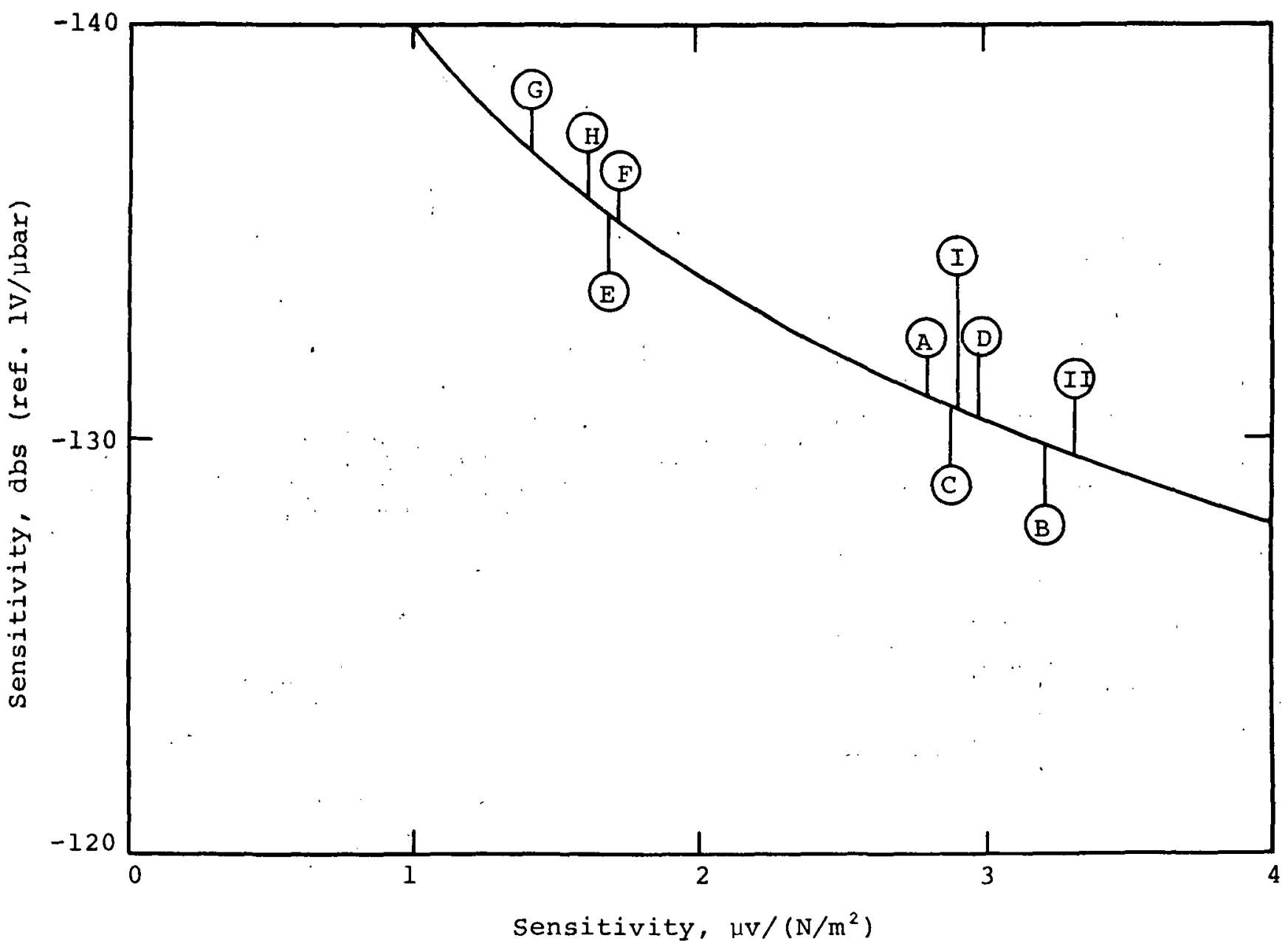

Figure 10.- Sensitivity of all pressure sensors in decibel units. 


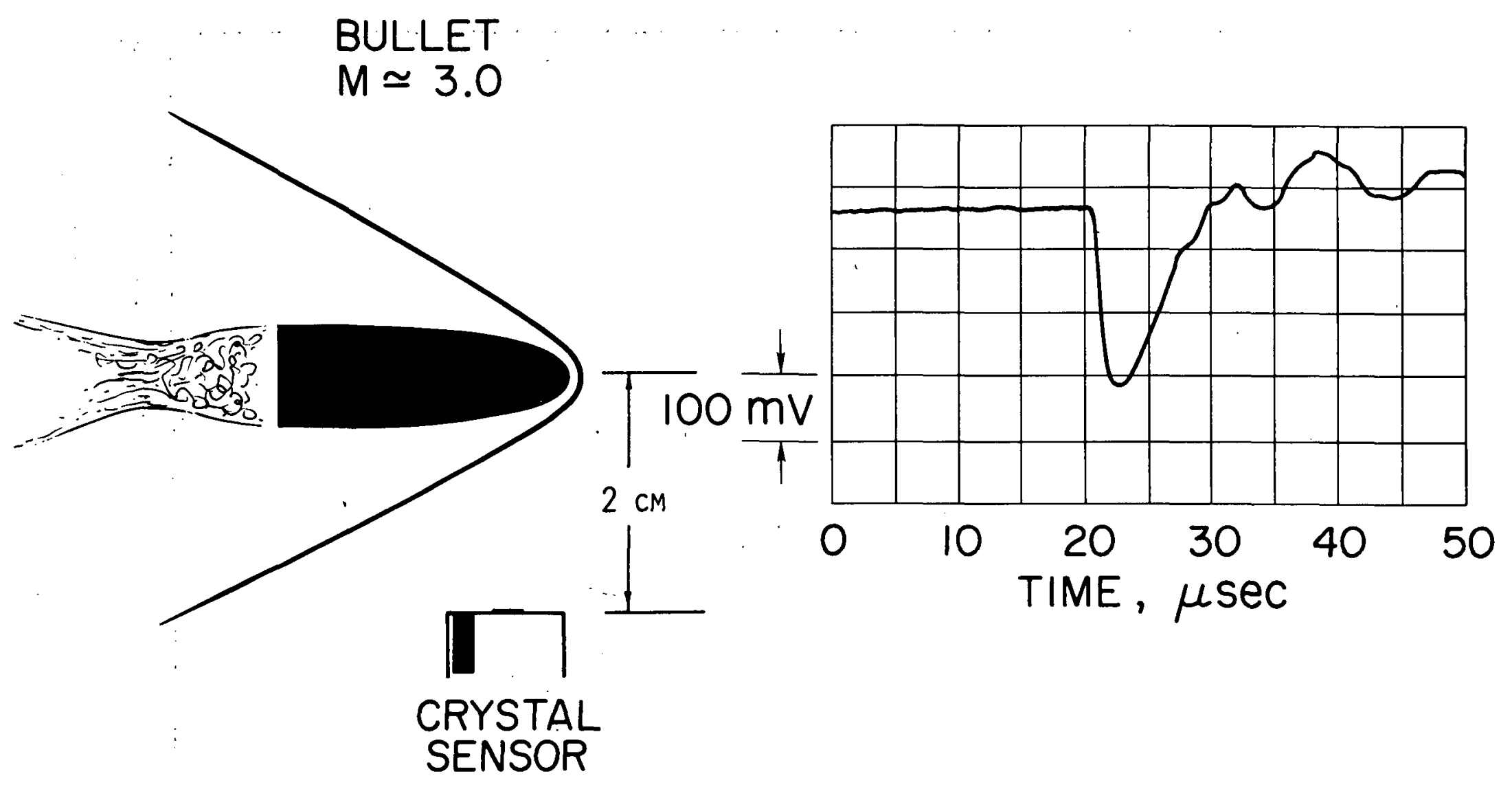

Figure 11.-Determination of time rise of crystal sensor. 


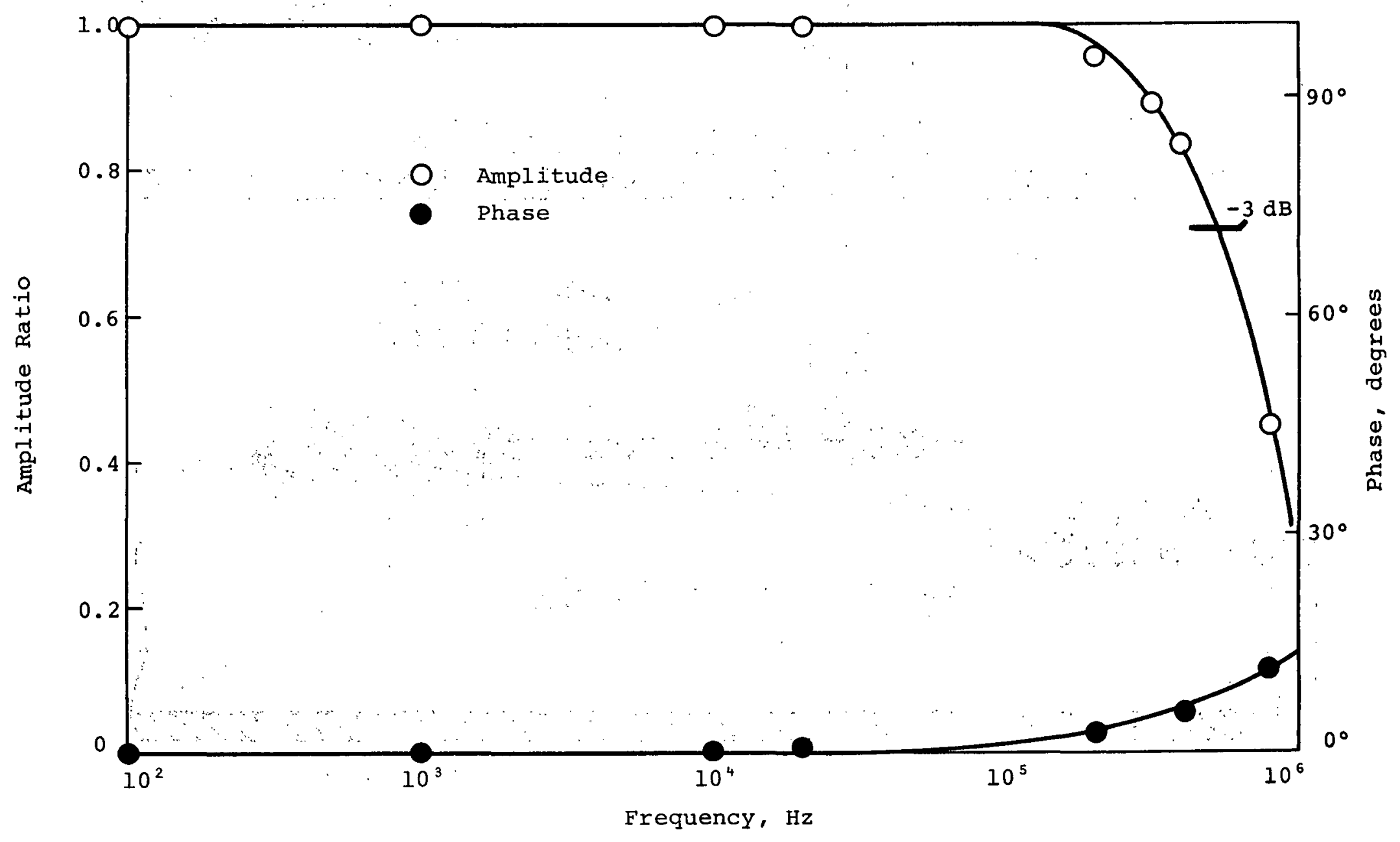

Figure 12.- Crystal sensor characteristics - amplitude and phase versus frequency. 


$$
M_{\infty}=5-10 \quad P_{R}=6.5 \times 10^{5}-11 \times 10^{6} \mathrm{~N} / \mathrm{m}^{2} \quad T_{R}=550-1400^{\circ} \mathrm{K}
$$

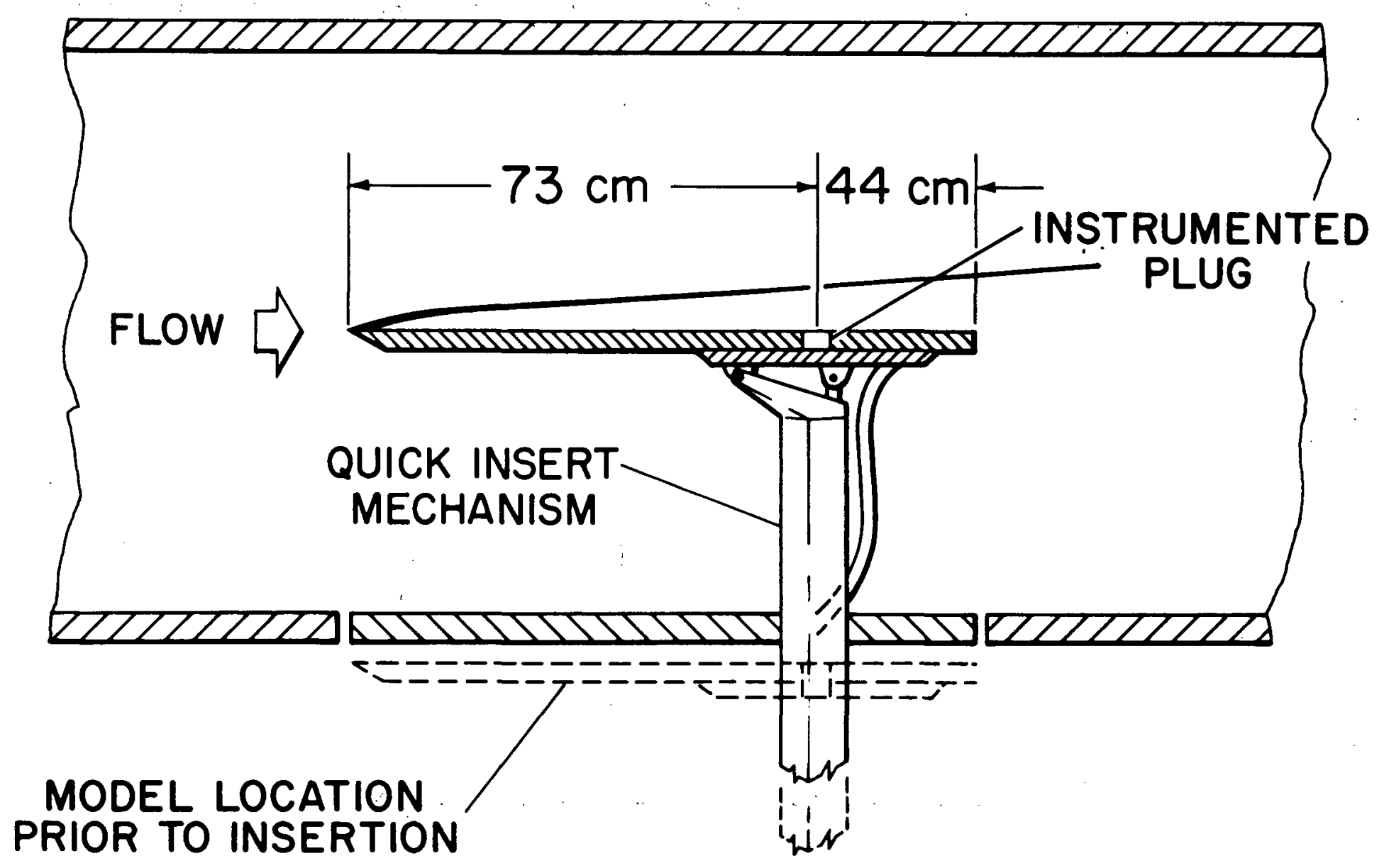

Figure 13.- Flat-plate model installed in Ames 3.5-Foot Hypersonic Wind Tunnel. 


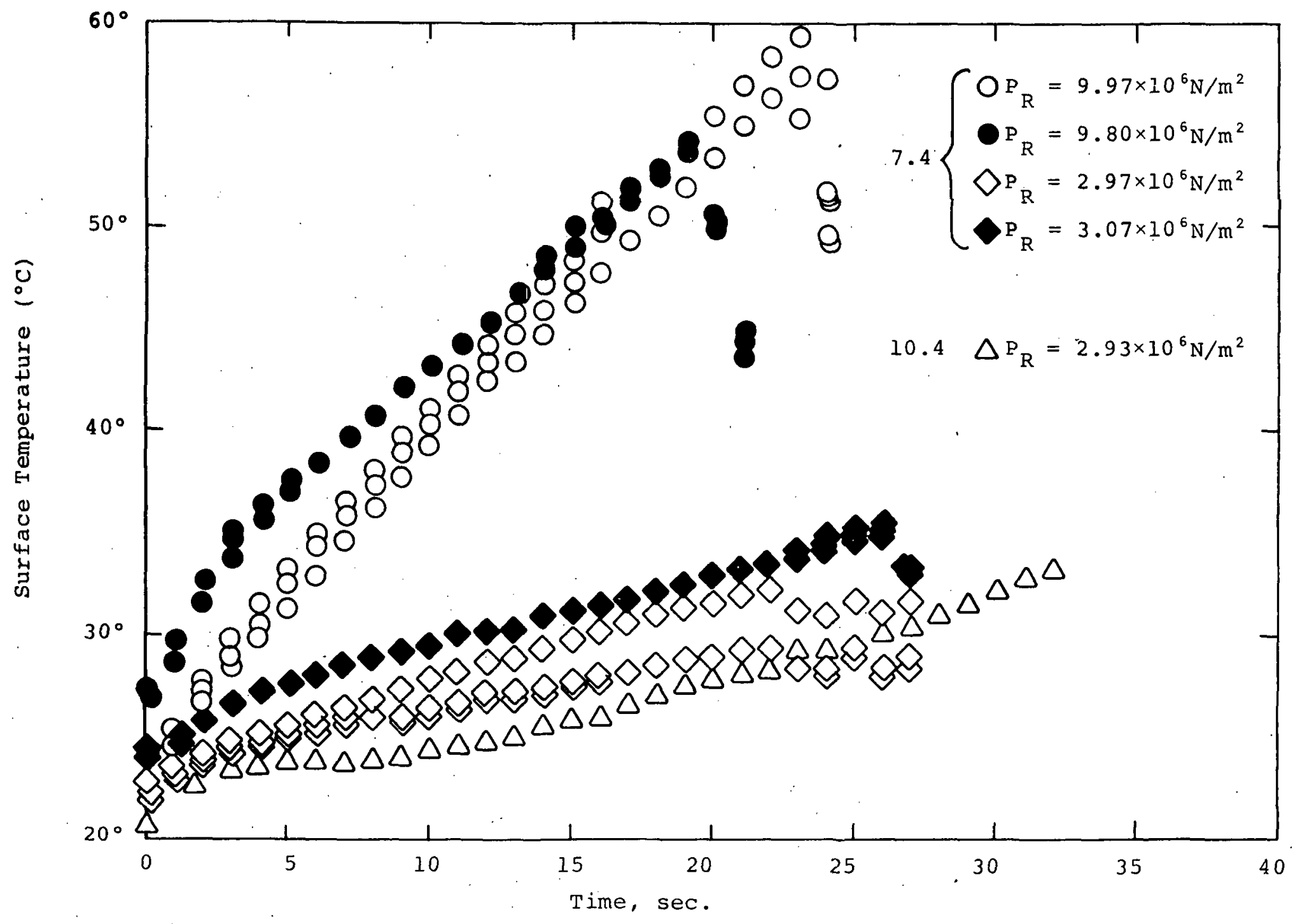

Figure 14.- Surface temperatures on flat-plate model during tests. 


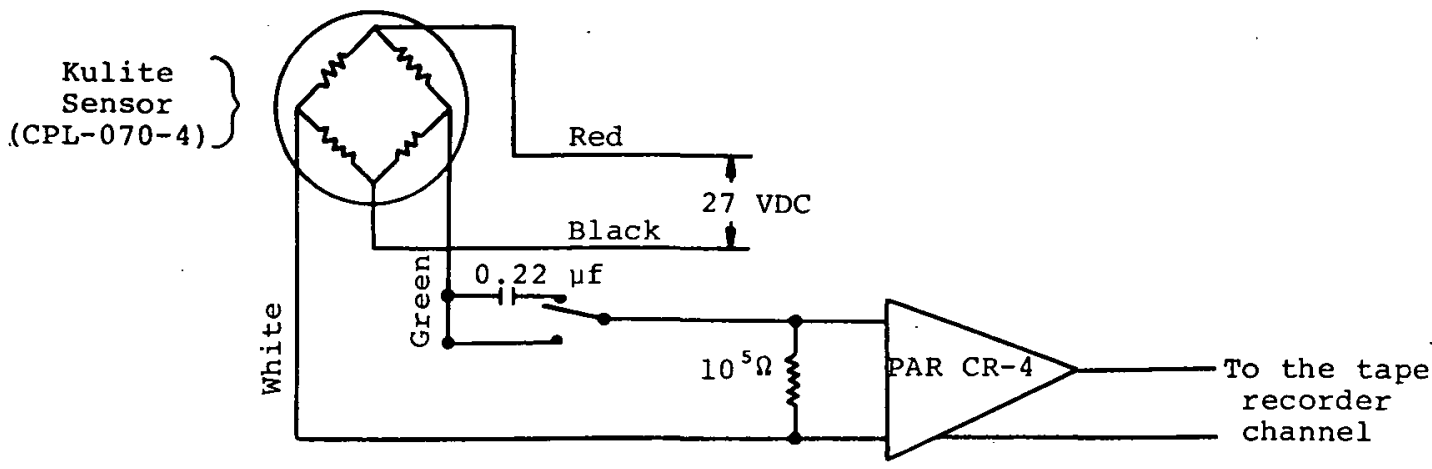

$0.22 \mu \mathrm{f}$ condenser for blocking DC voltage.

$10^{5} \Omega$ leakage path resistance for charge build-up in condenser.

PAR CR-4 amplifier operation-single ended.

(a) Schematic wiring diagram for Kulite sensor for unsteady pressure measurement.

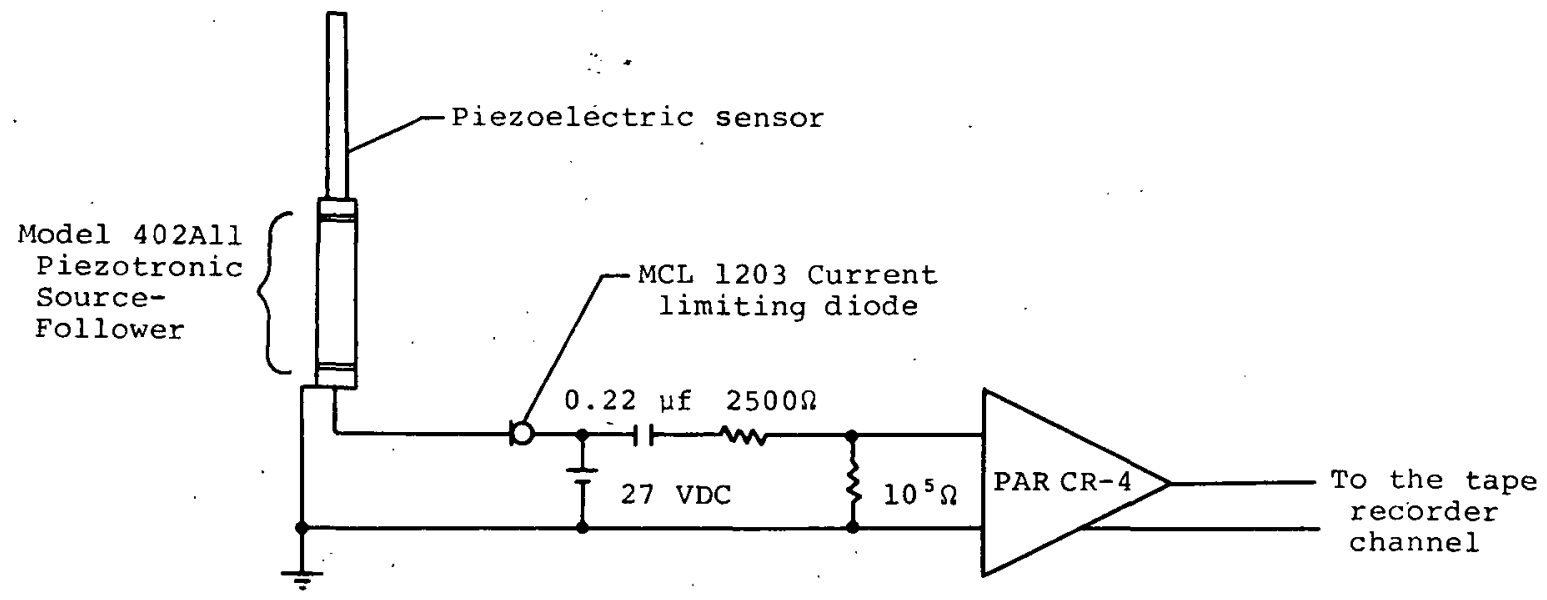

$0.22 \mu f$ - DC blockage capacitance.

MCL 1302 - 2 ma constant current diode.

$2500 \Omega$ - resistance used in order to obtain less than $1 \mathrm{db}$ noise figure from $\mathrm{CR}-4$ amplifier.

(b) Schematic wiring diagram for crystal sensor.

Figure 15.- Electrical circuit used for pressure sensors. 


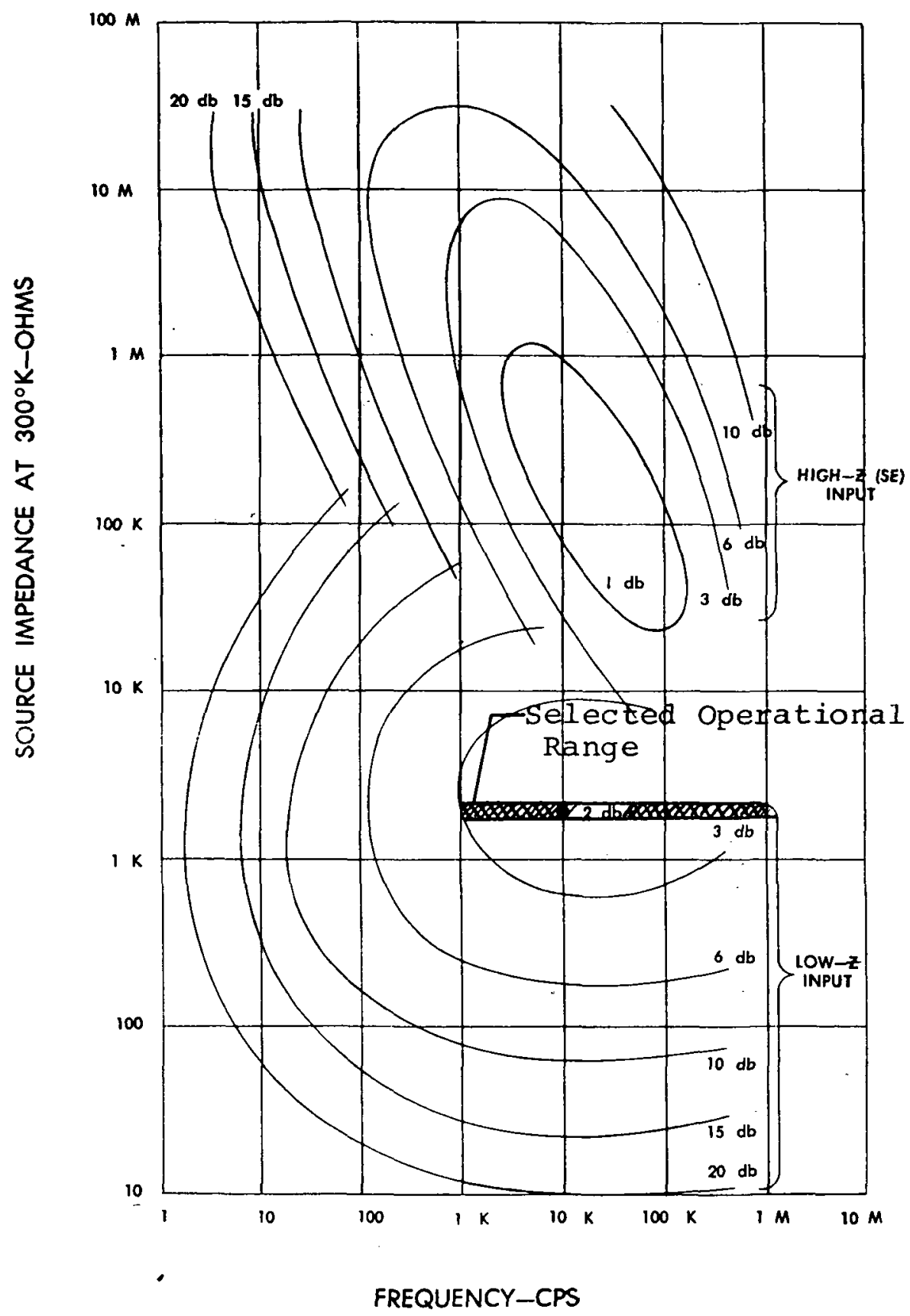

Noise Figure $(F)=20 \log \frac{\mathrm{S} / \mathrm{N} \text { input voltage }}{\mathrm{S} / \mathrm{N} \text { output voltage }}, \mathrm{dB}$

Figure 16.- Typical amplifier noise figure contours and the selected operational range. 


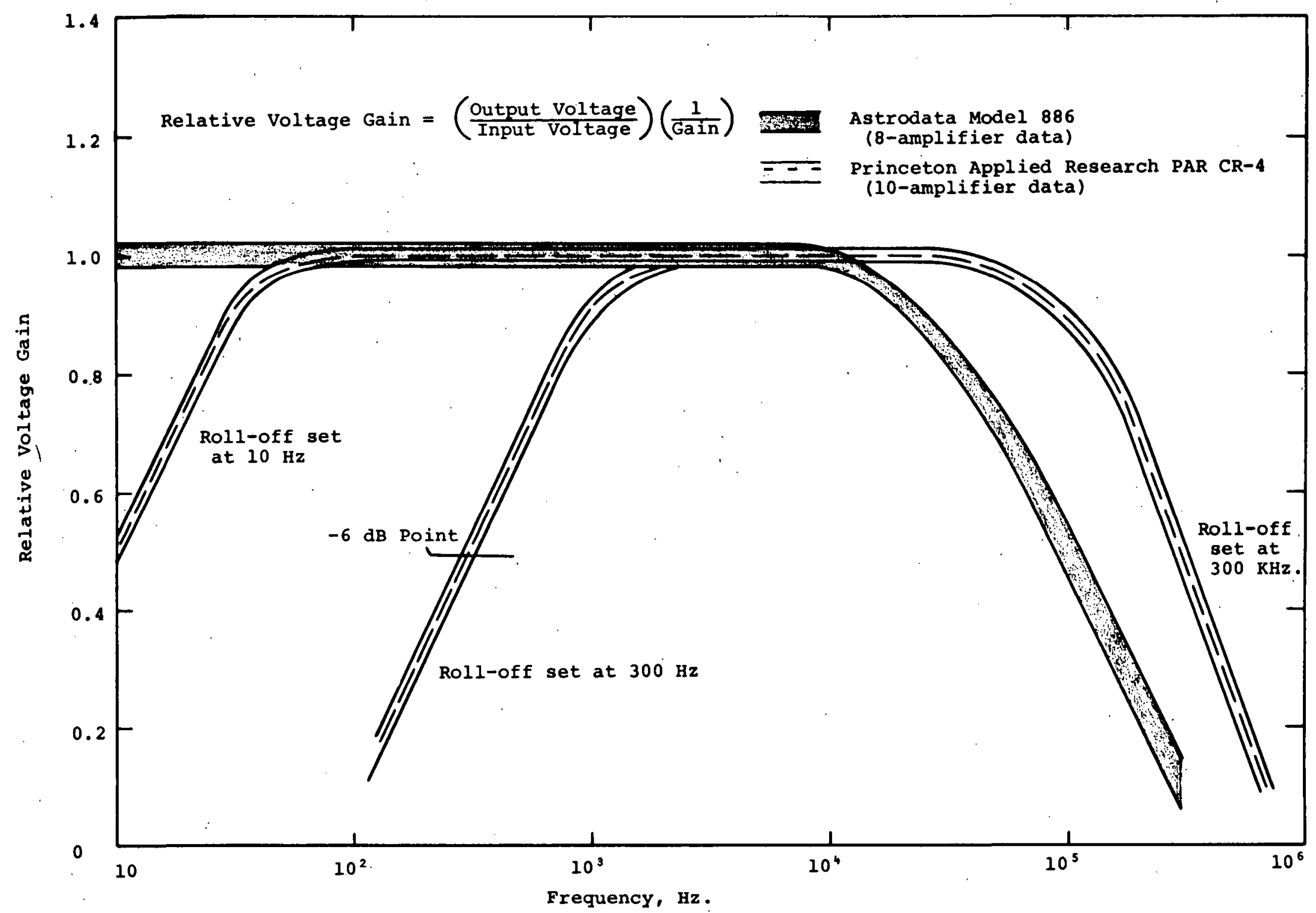

Figure 17.- Amplifier frequency response characteristics. 

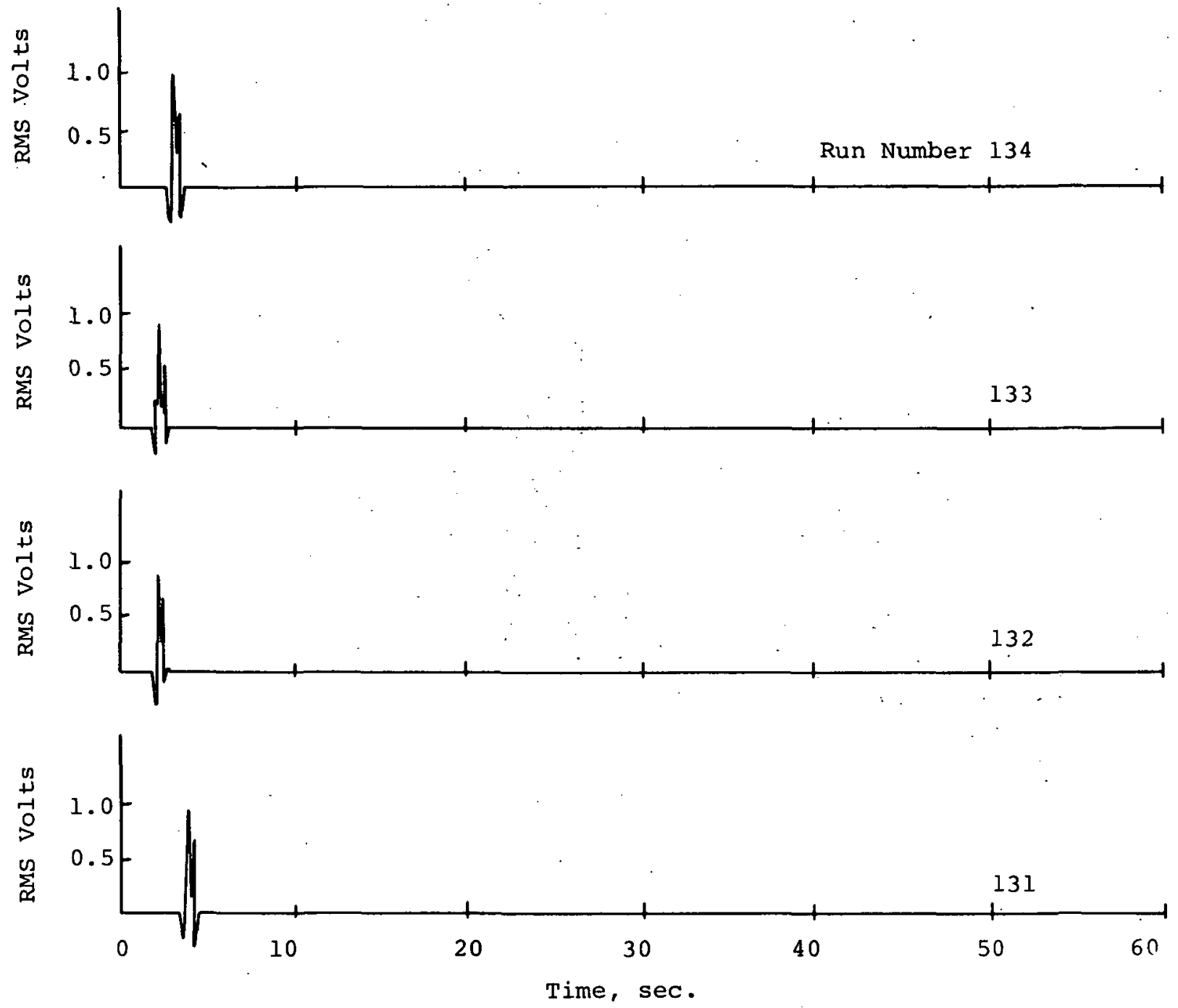

Figure 18.- Typical results for the acceleration of test model. 


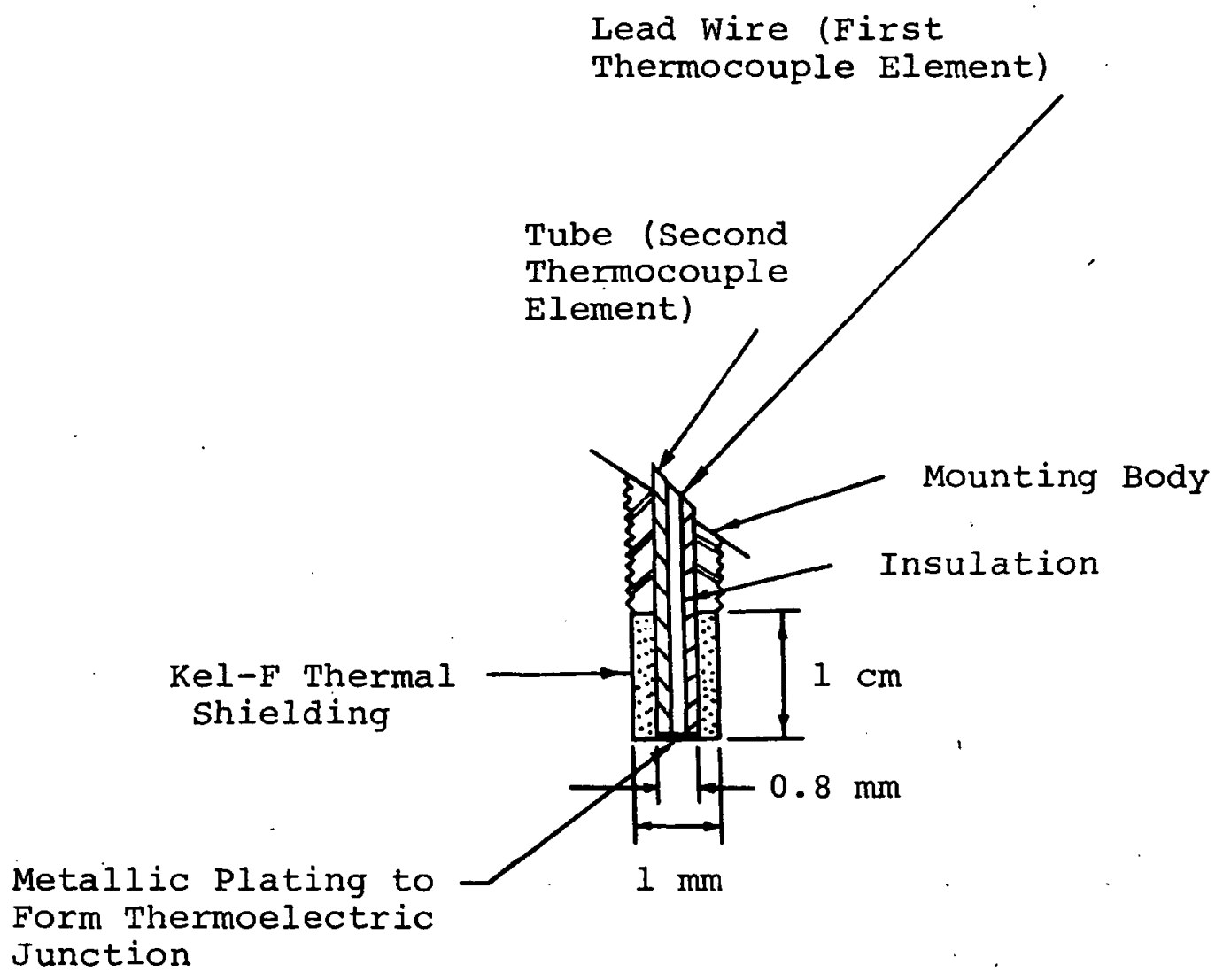

Figure 19.- Cross-sectional schematic of TCS Series Probe. 


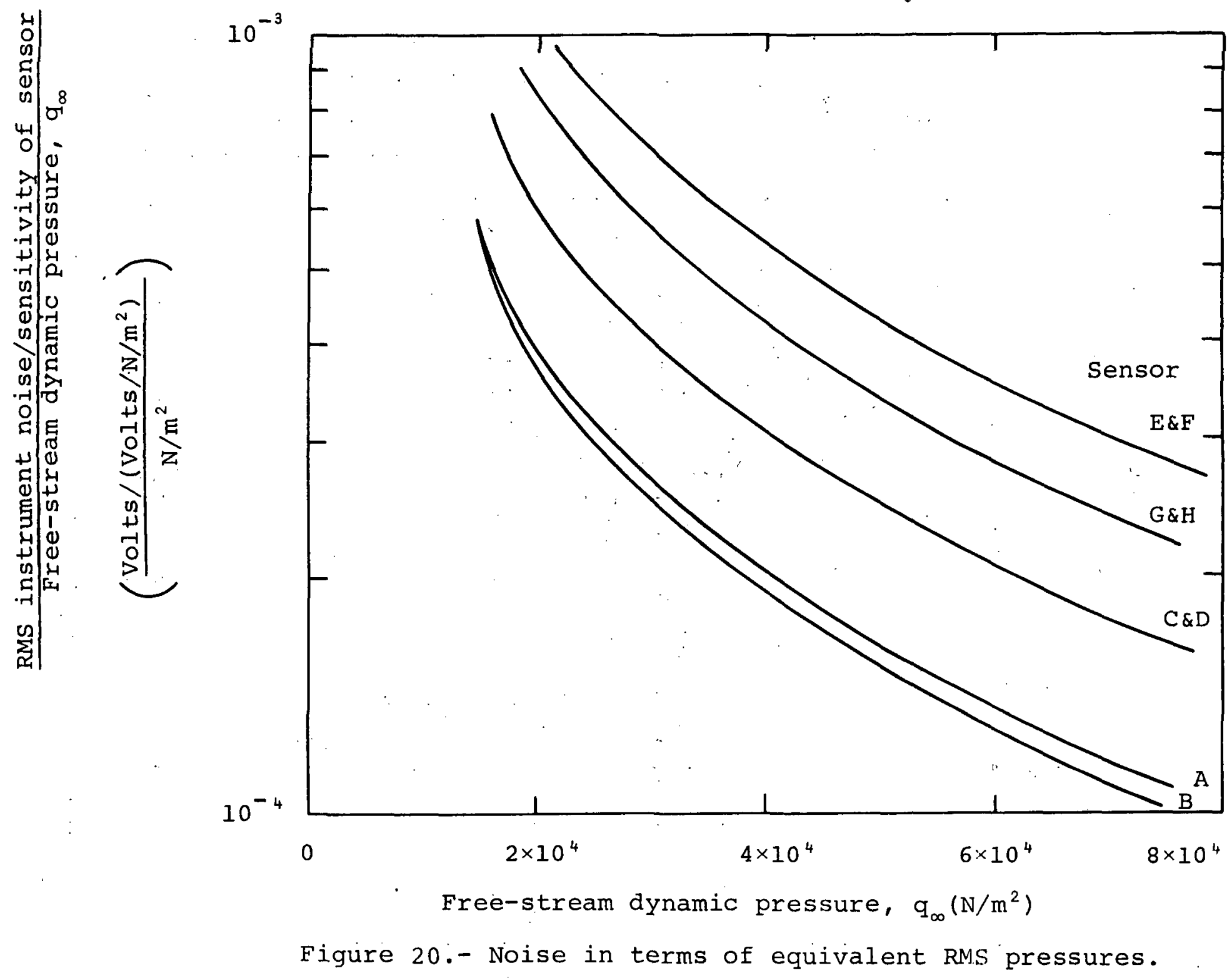




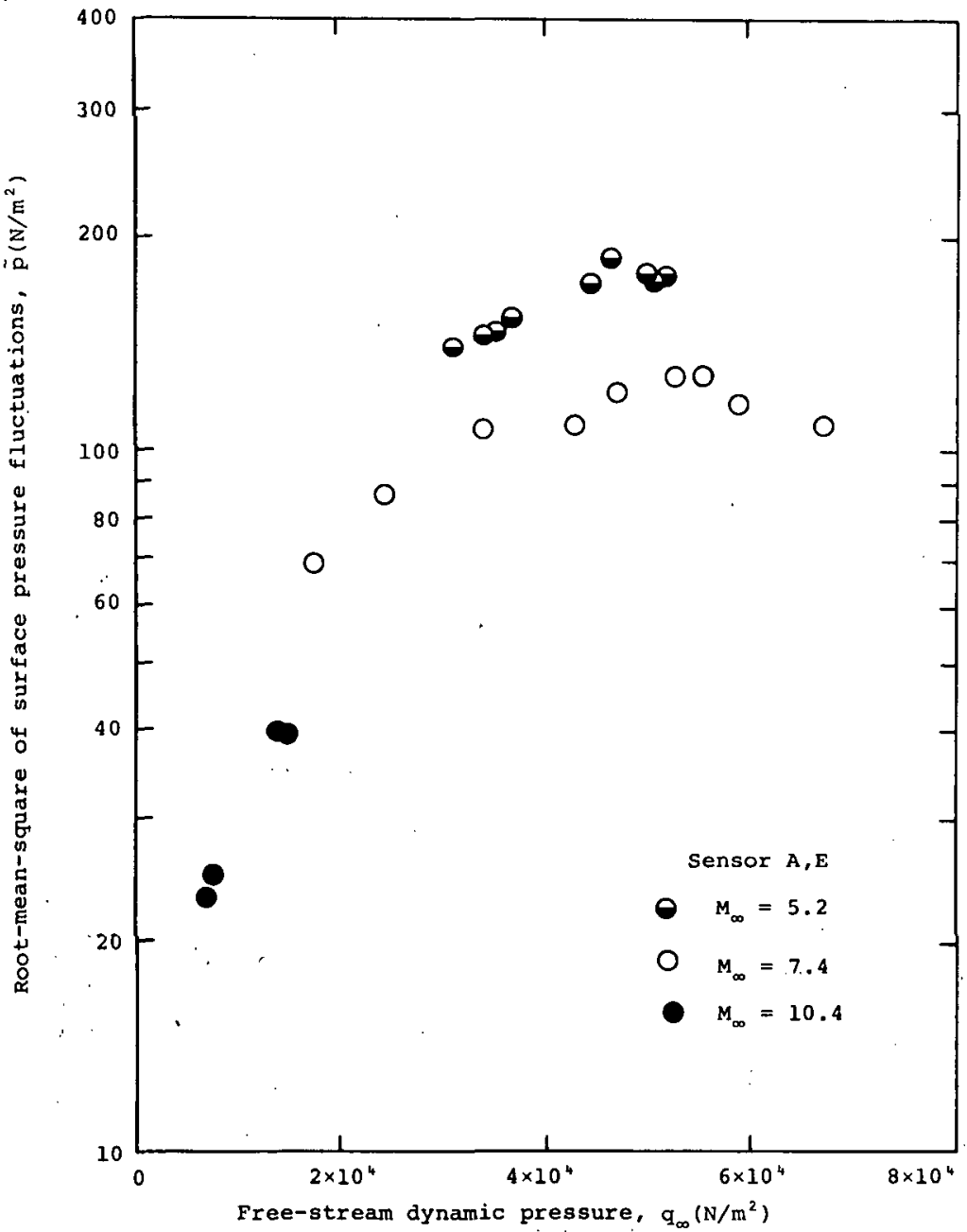

(a) Pressure data from Sensor $A, E$.

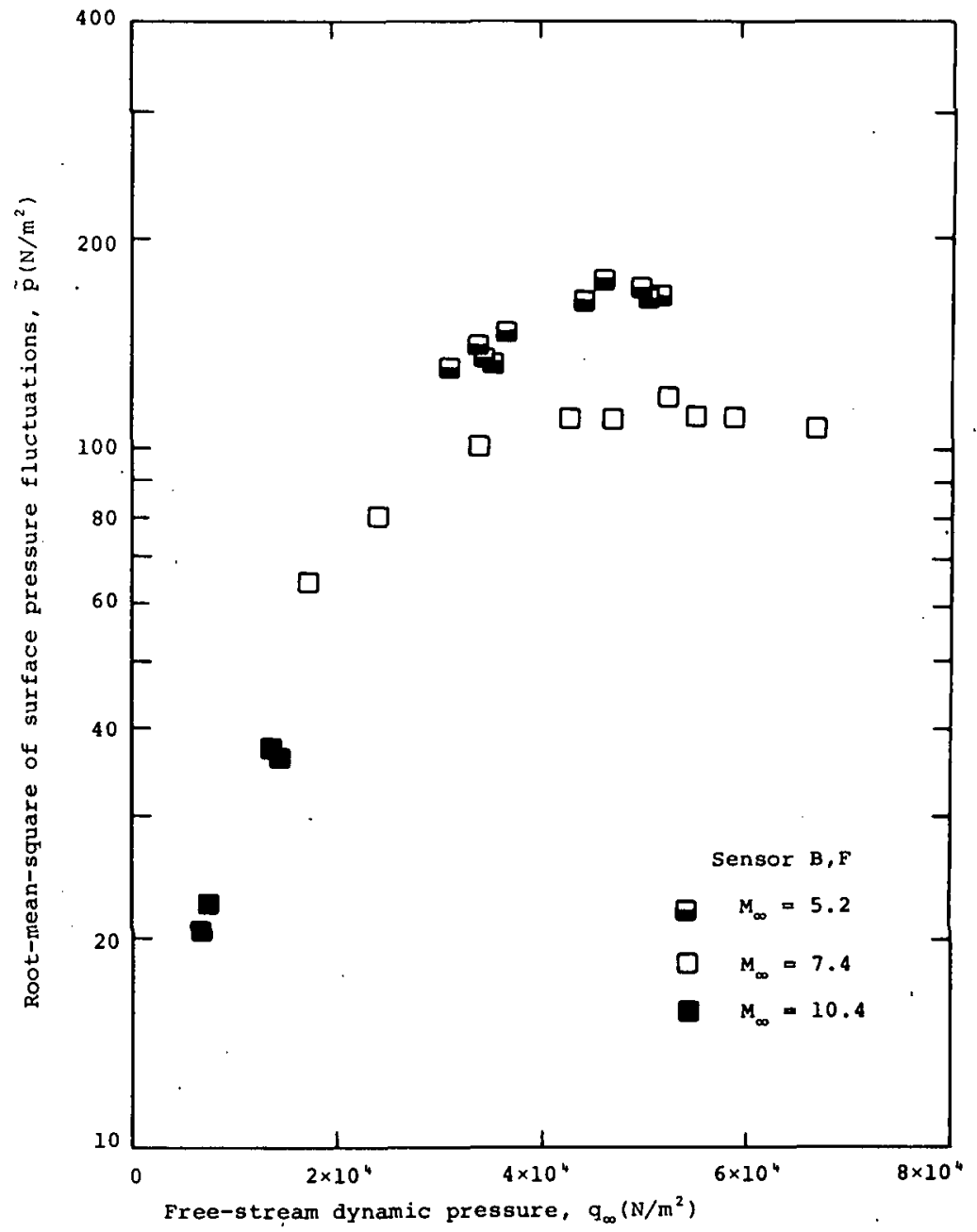

(b) Pressure data from sensor B,F.

Figure 2l.- RMS surface pressure against dynamic pressure. 


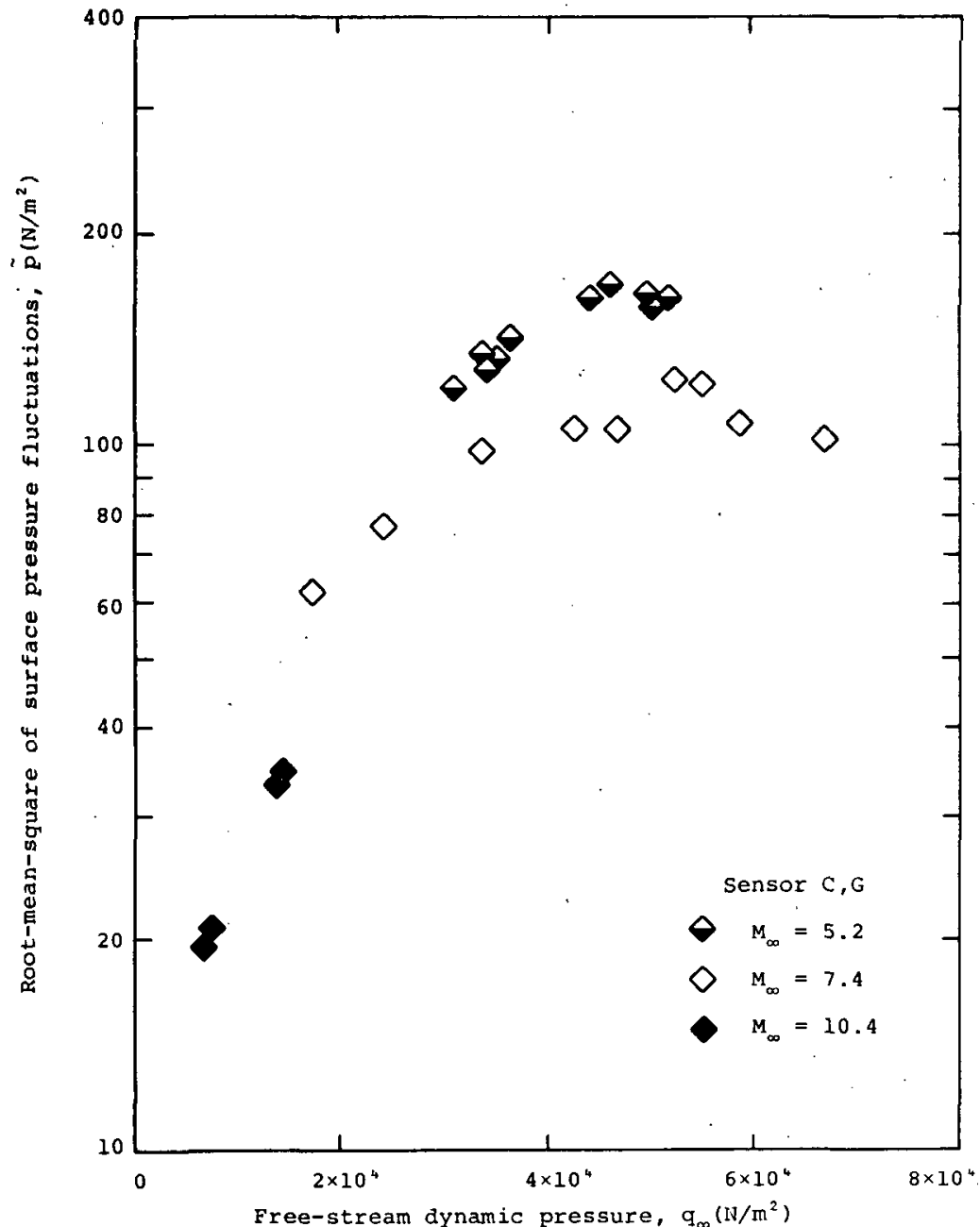

(c) Pressure data from sensor C,G.

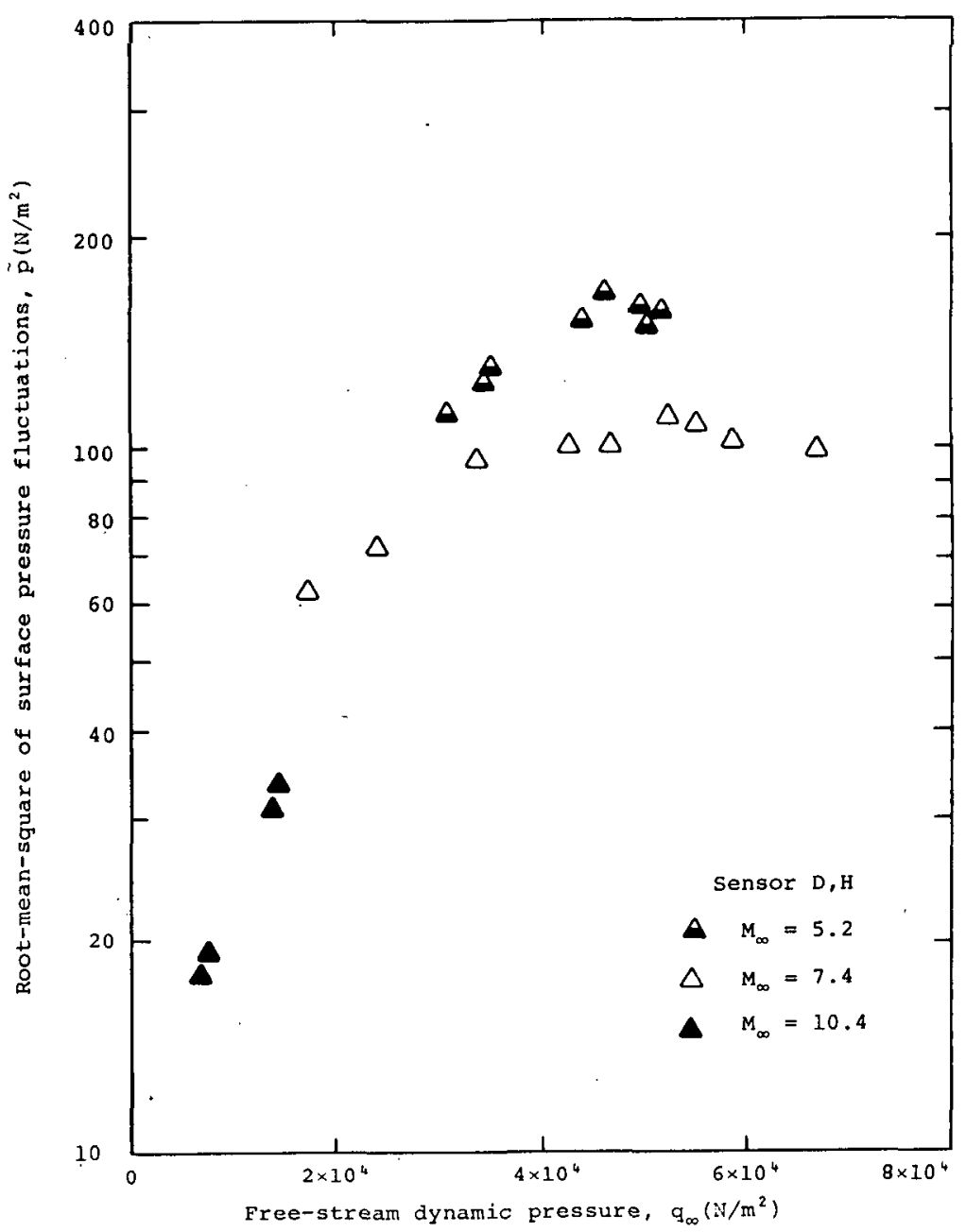

(d) Pressure data from sensor $\mathrm{D}, \mathrm{H}$.

Figure 21.- Concluded. 


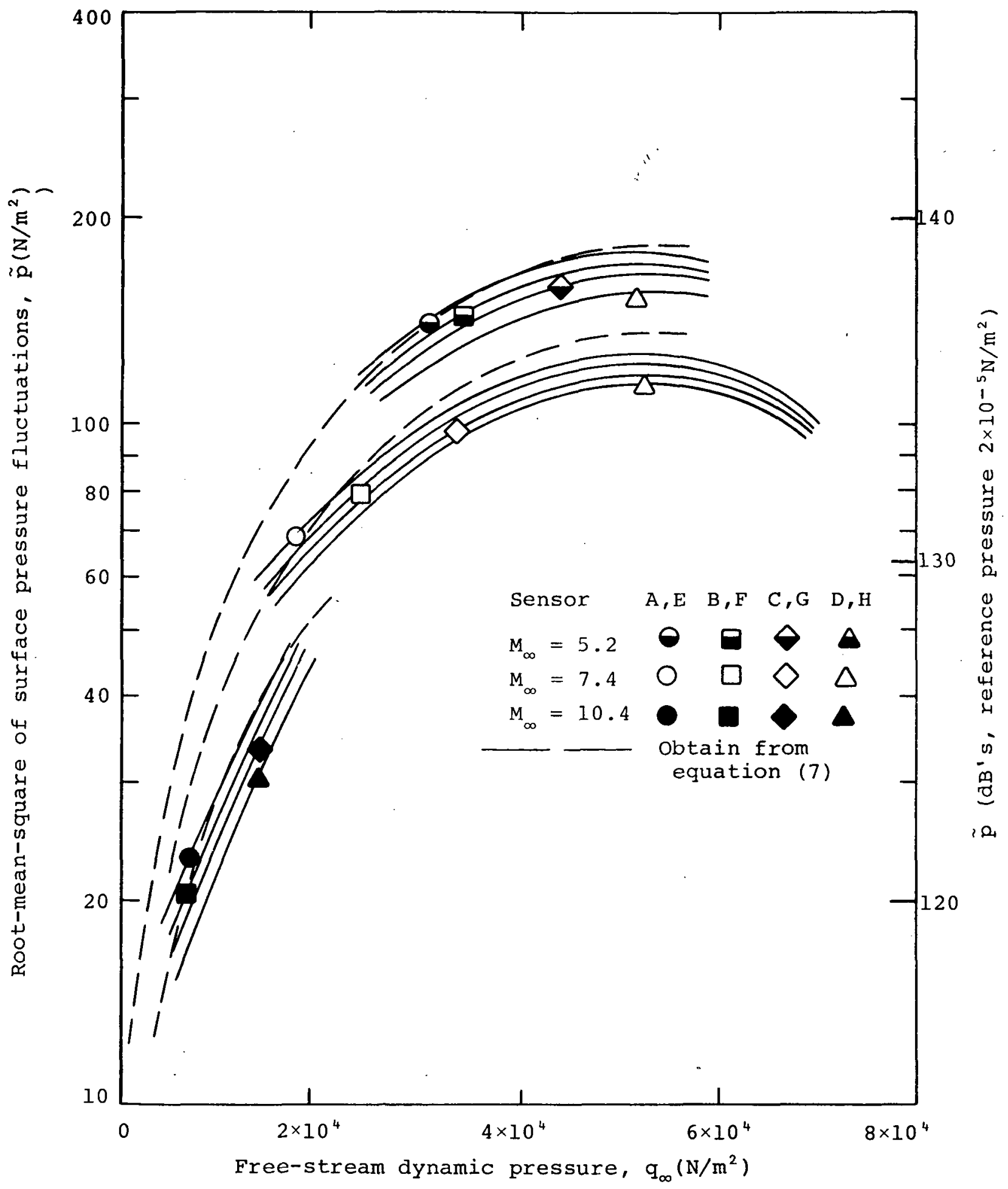

Figure 22.- Composite plot of RMS pressure against dynamic pressure. 


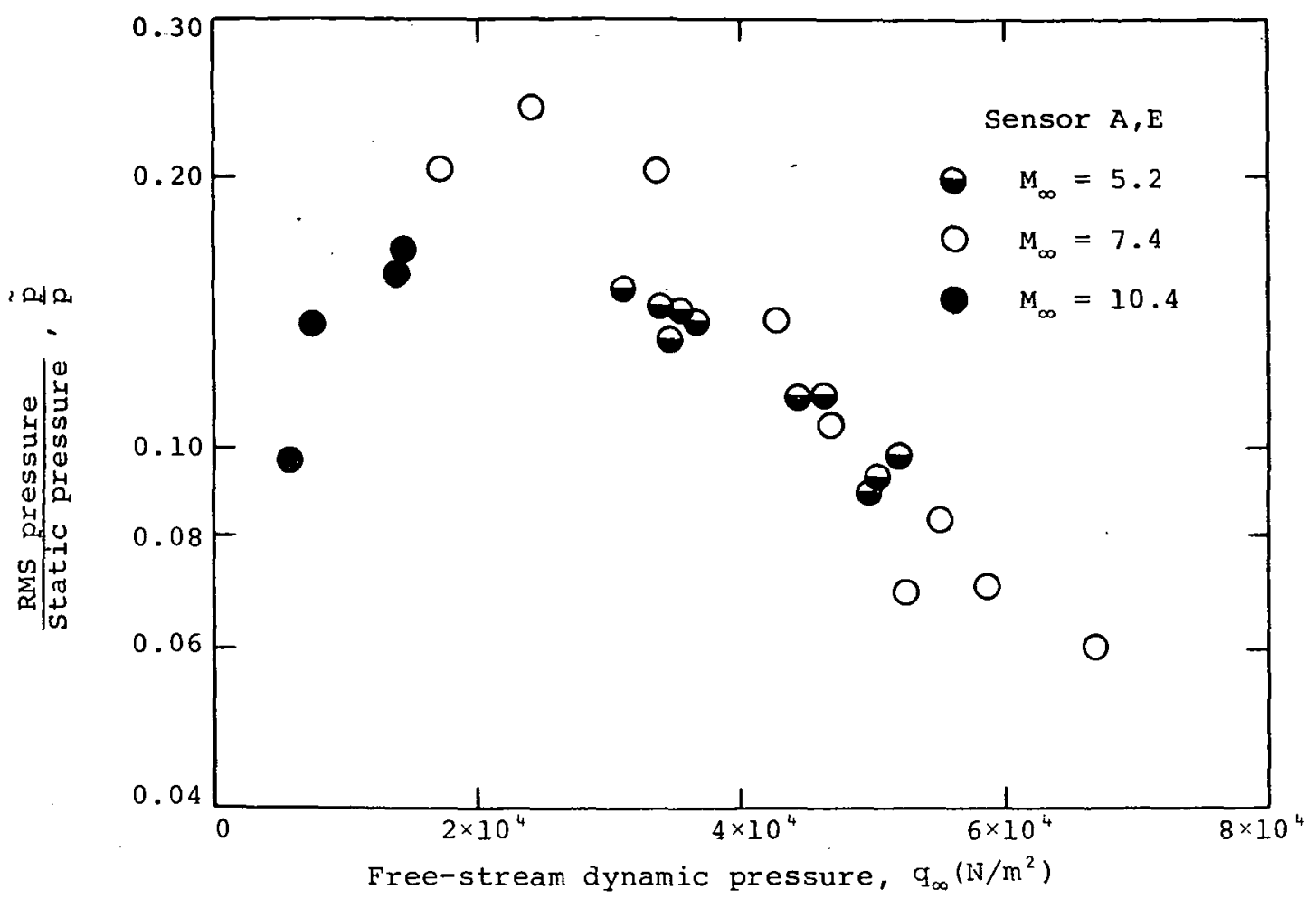

(a) Pressure data from sensor A,E.

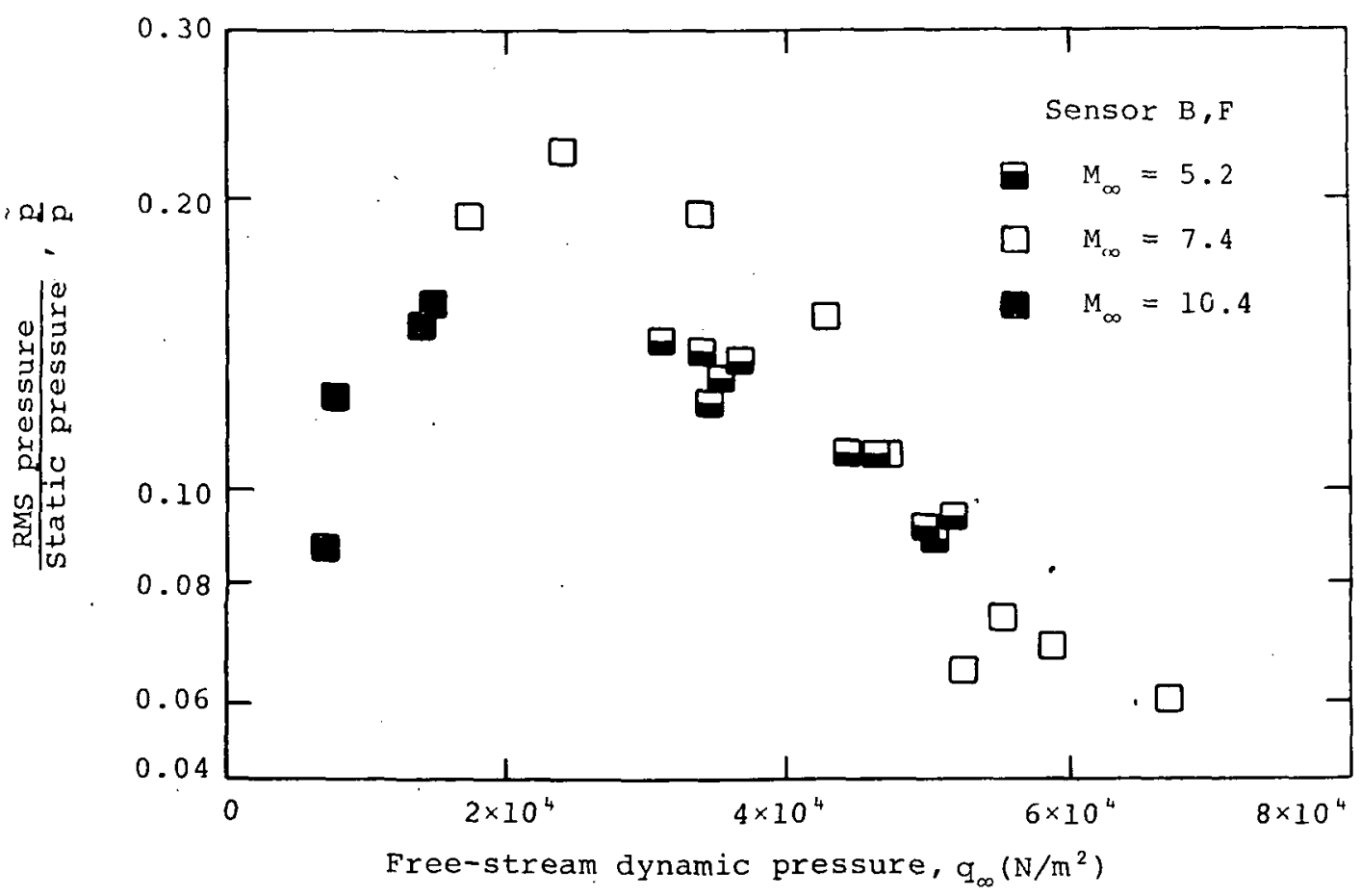

(b) Pressure data from sensor B,F.

Figure 23.- Ratio of RMS pressure to mean static pressure against dynamic pressure. 


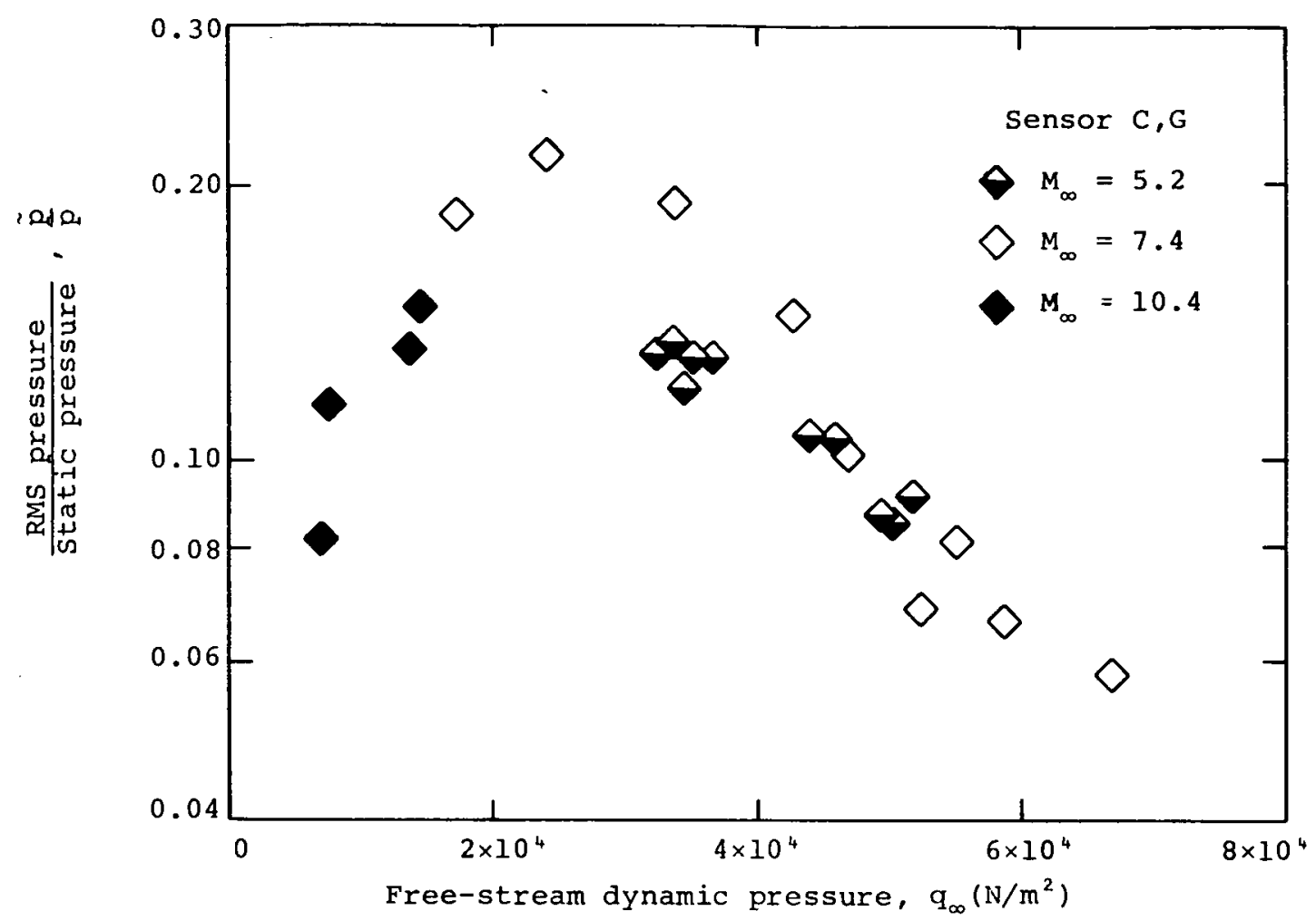

(c) Pressure data from sensor C, G.

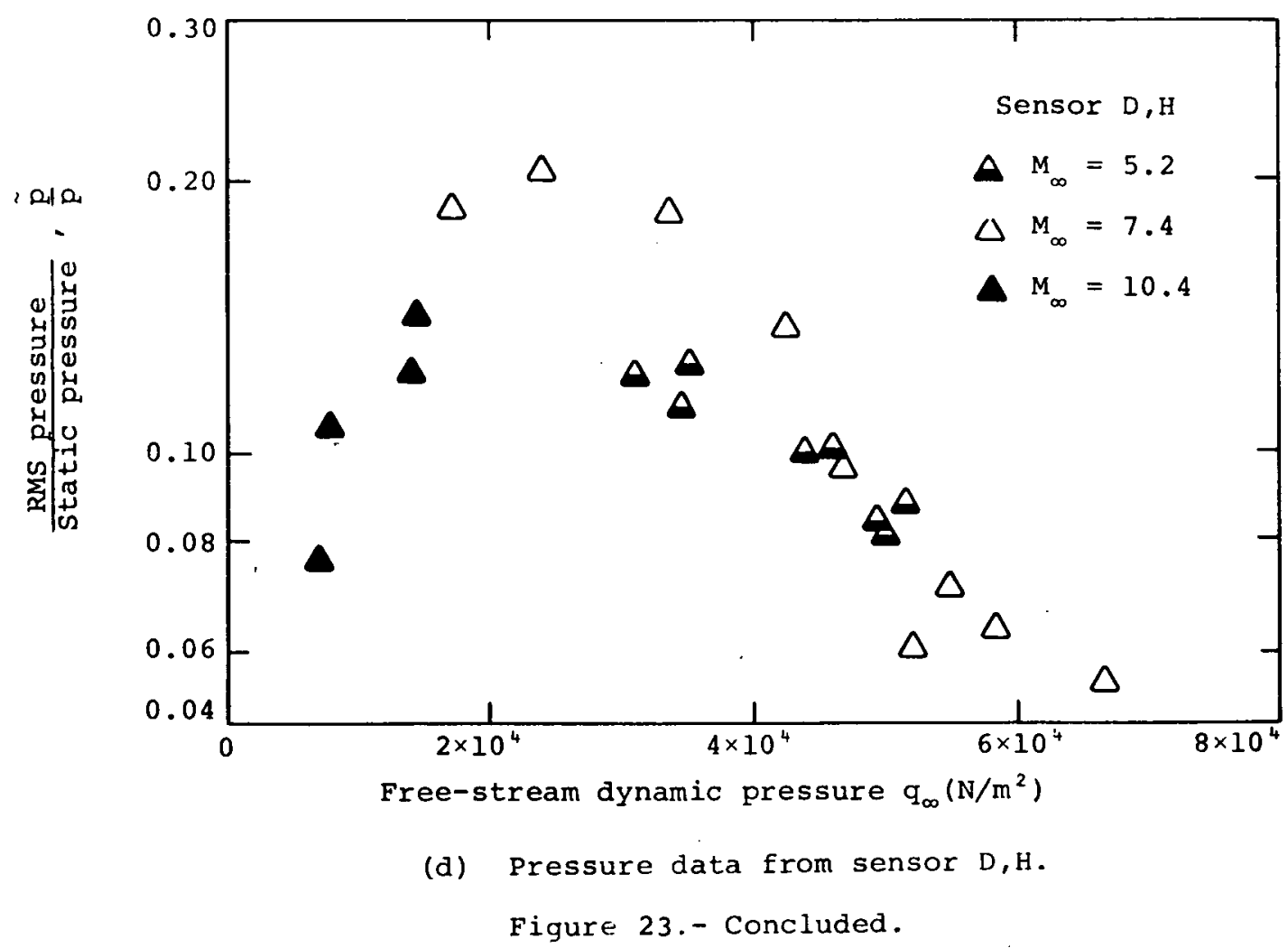




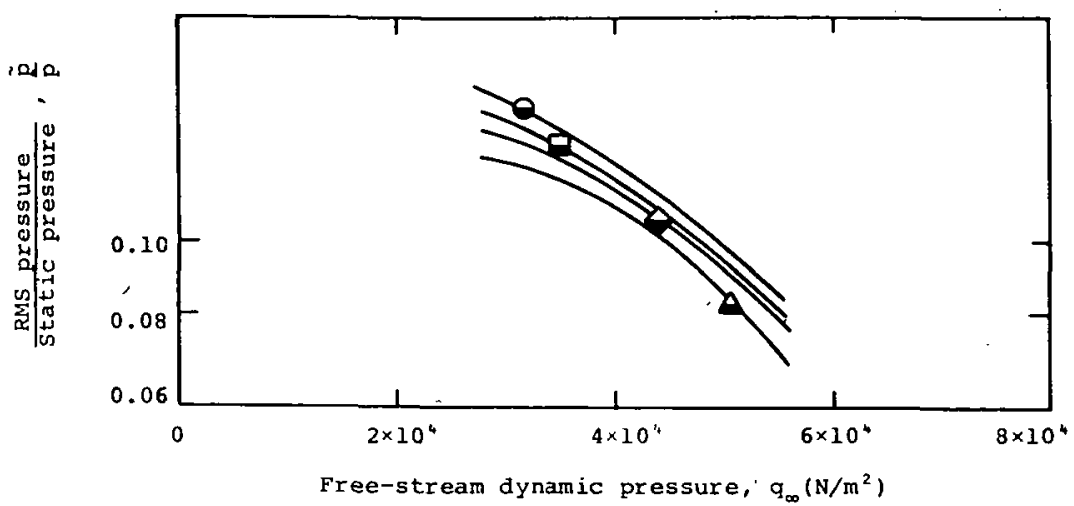

(a) Data for 5.2 ilach number tests.

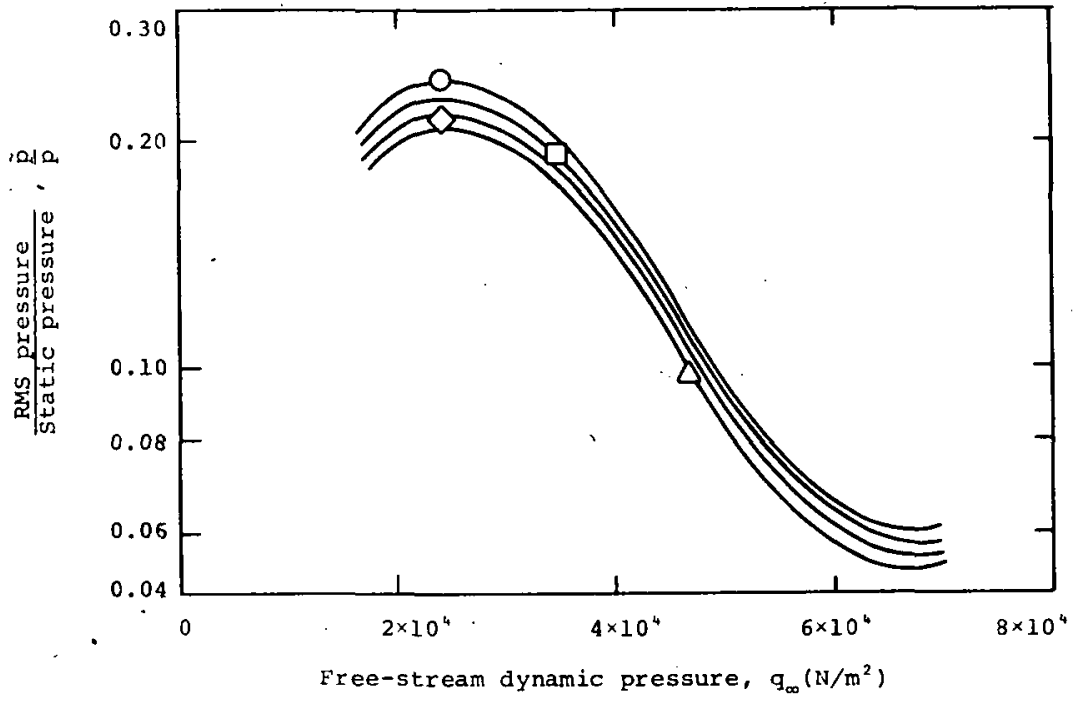

(b) Data for 7.4 Mach number tests.

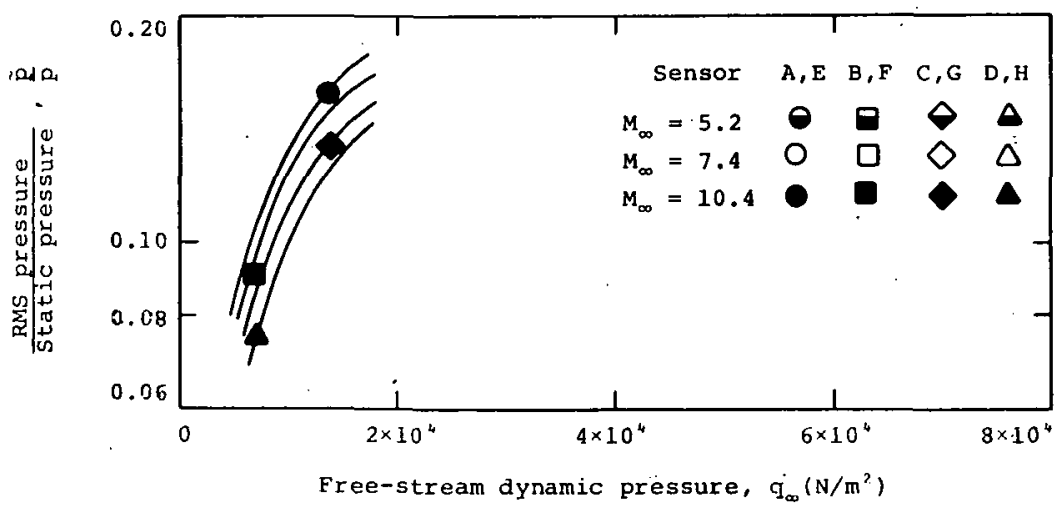

(c) Data for 10.4 Mach number tests.

Pigure 24.- Composite plot of the ratio of RMS pressure to mean static pressure against dynamic pressure. 


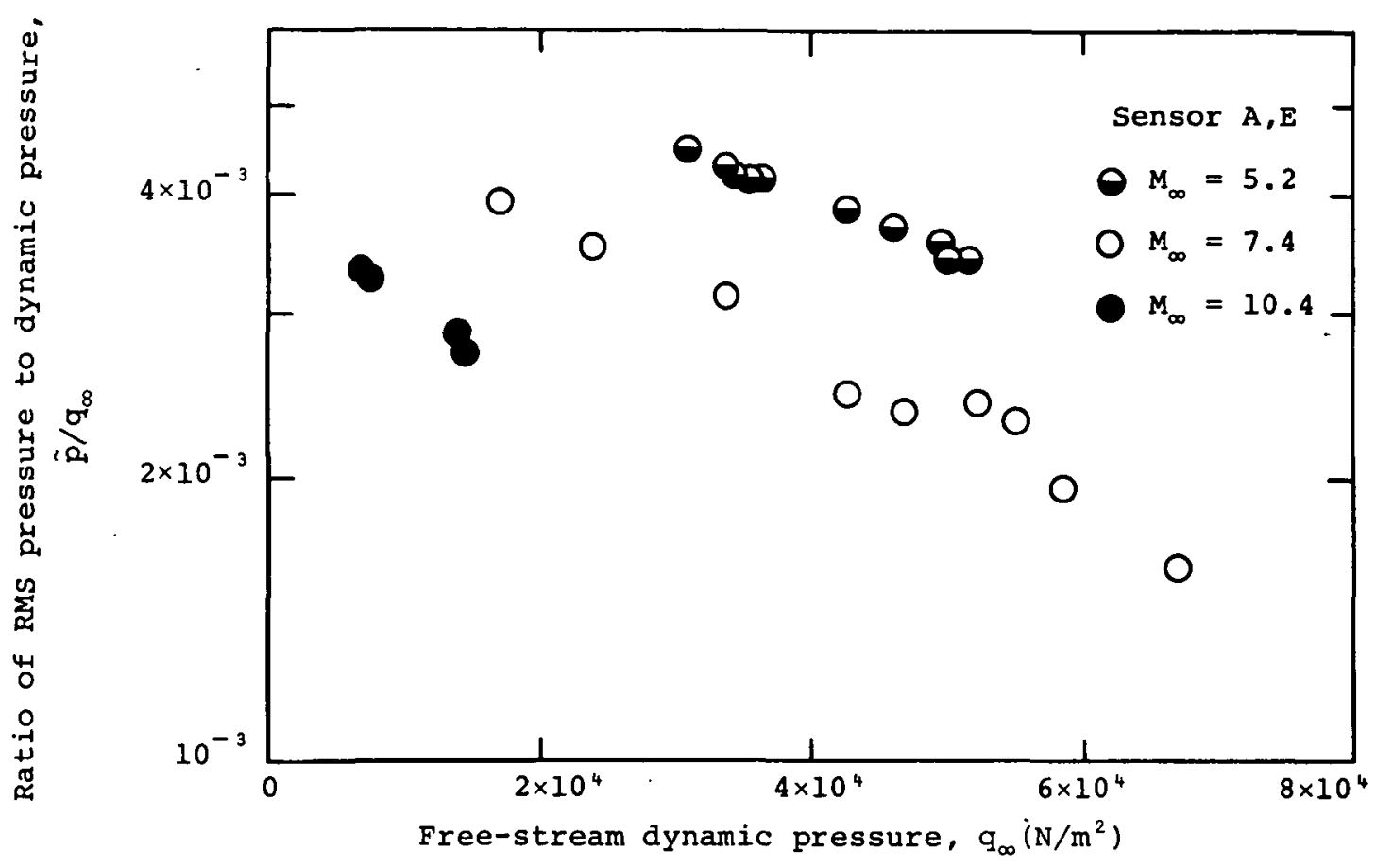

(a) Pressure data from sensor A,E.

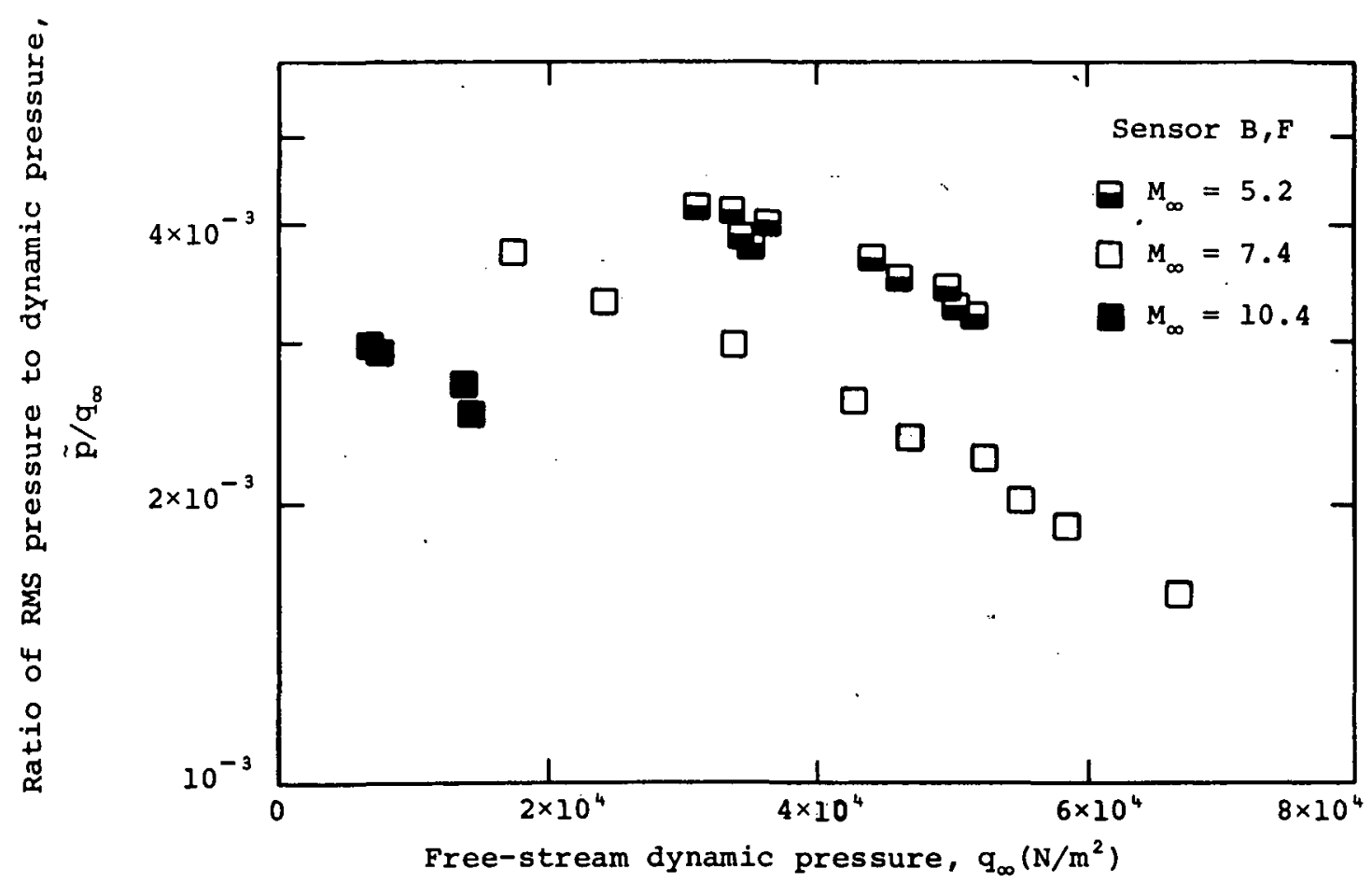

(b) Pressure data from sensor $B, F$.

Figure 25.- Ratio of RMS pressure to dynamic pressure, $\tilde{p} / q_{\infty}$ against dynamic pressure, $q_{\infty}$. 


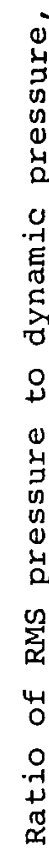

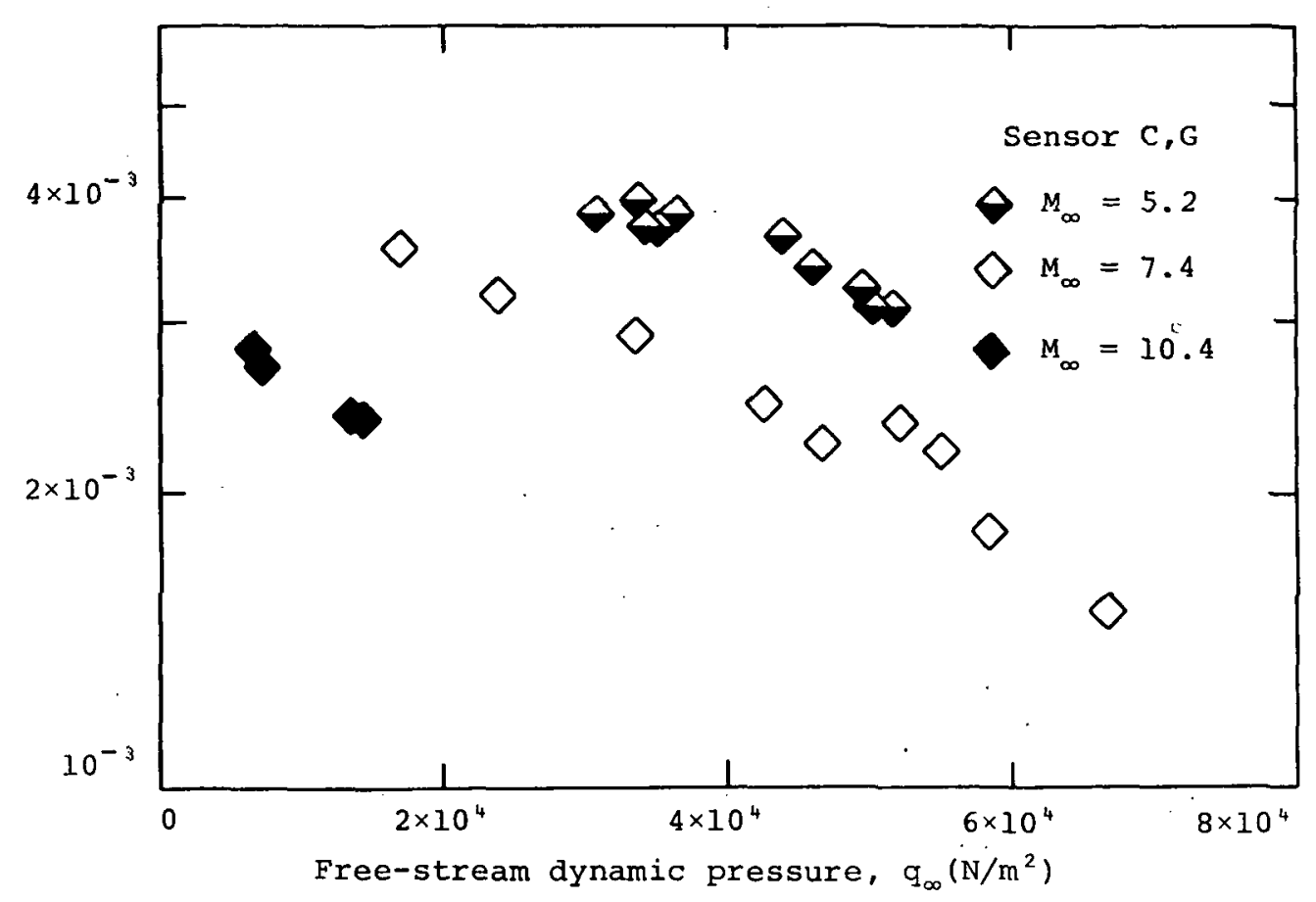

(c) Pressure data from sensor C,G.

0

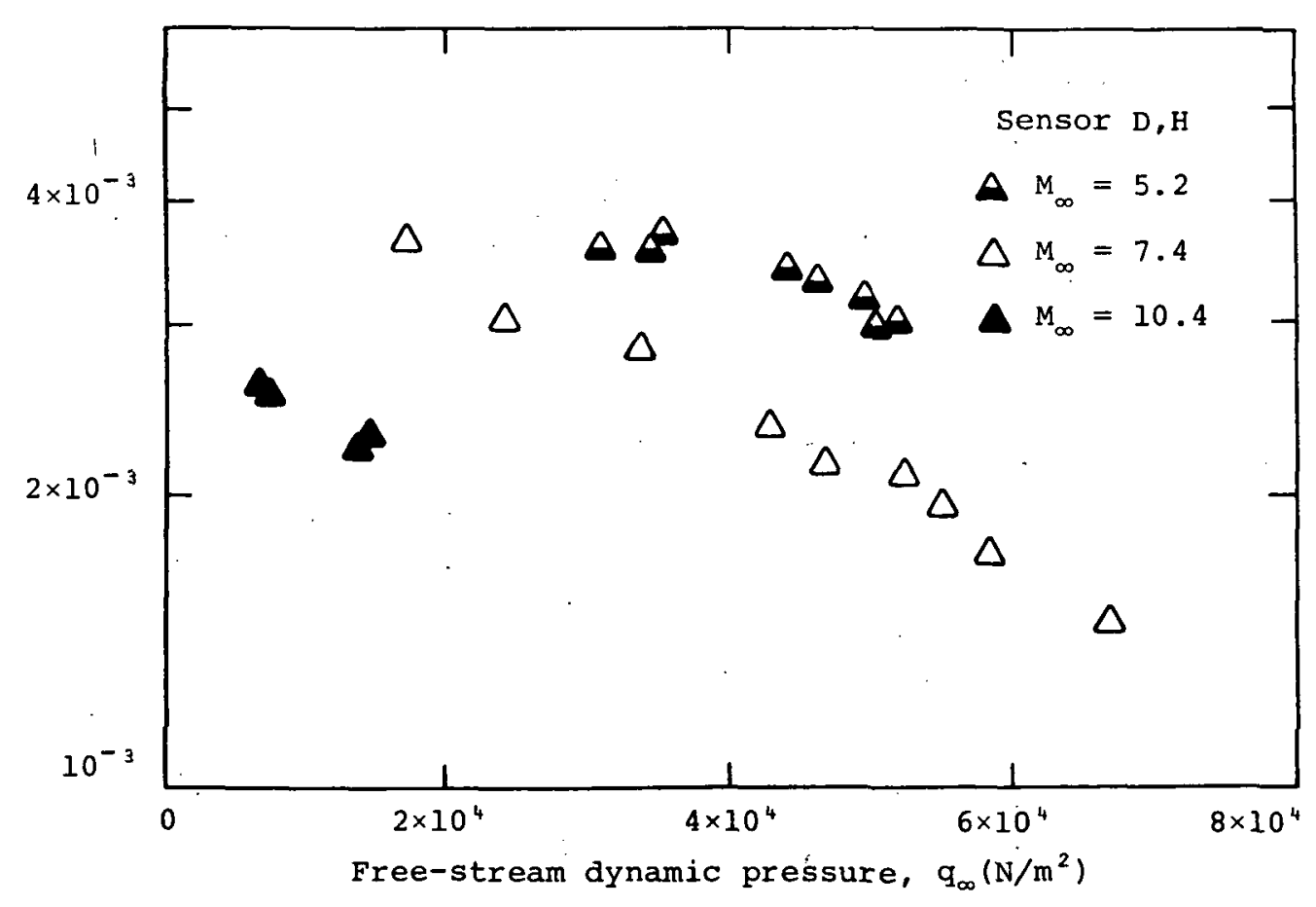

(d) Pressure data from sensor D, H.

Figure 25.- Concluded. 

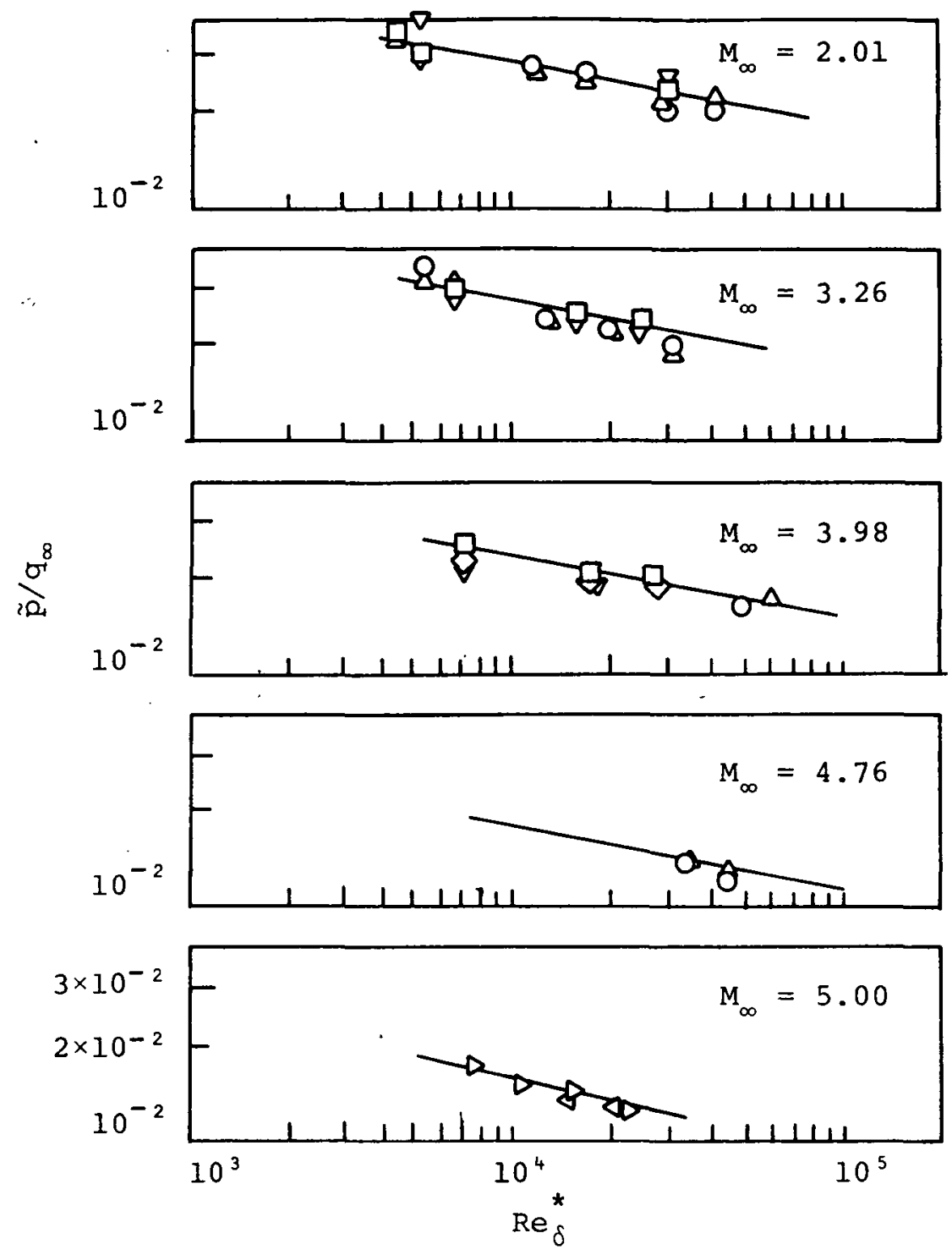

\begin{tabular}{|l|l|l|l|}
\hline SYMBOL & CRYSTAL & D, in. & STATION \\
\hline$O$ & A & 0.015 & 24 \\
$\triangle$ & B & 0.03 & 24 \\
$\square$ & 4 & 0.015 & 13 \\
$\bigcirc$ & 5 & 0.015 & 13 \\
$\nabla$ & 6 & 0.03 & 13 \\
$D$ & 7 & 0.03 & 13 \\
$\nabla$ & 8 & 0.03 & 13 \\
\hline
\end{tabular}

Figure 26.- Data from reference 21, $\tilde{\mathrm{p}} / \mathrm{q}_{\infty}$ against $R e_{\delta}{ }^{\prime}$ for flat-plate. 


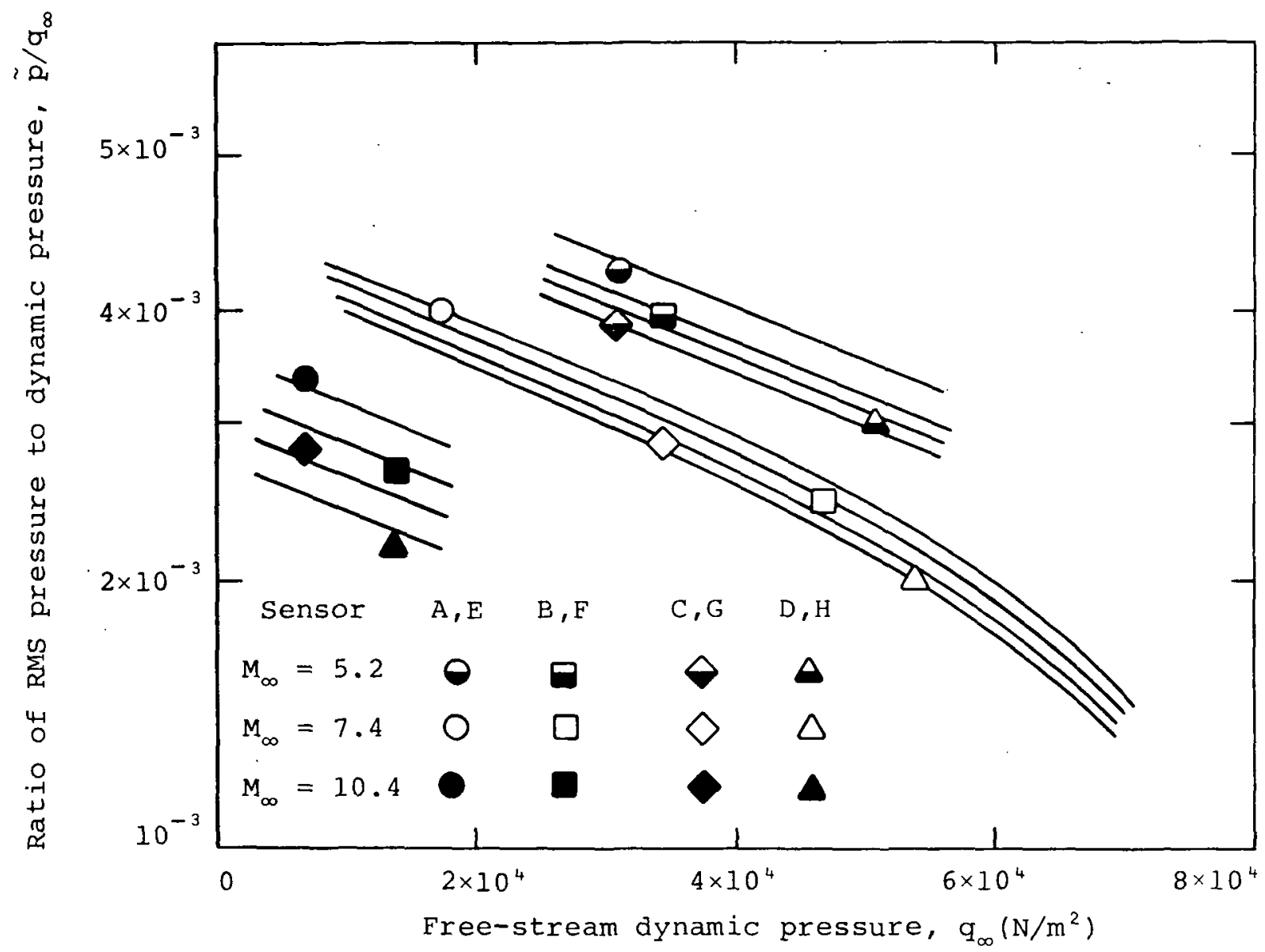

Figure 27.- Composite plot $\tilde{\mathrm{p}} / \mathrm{q}_{\infty}$ against $\mathrm{q}_{\infty}$. 


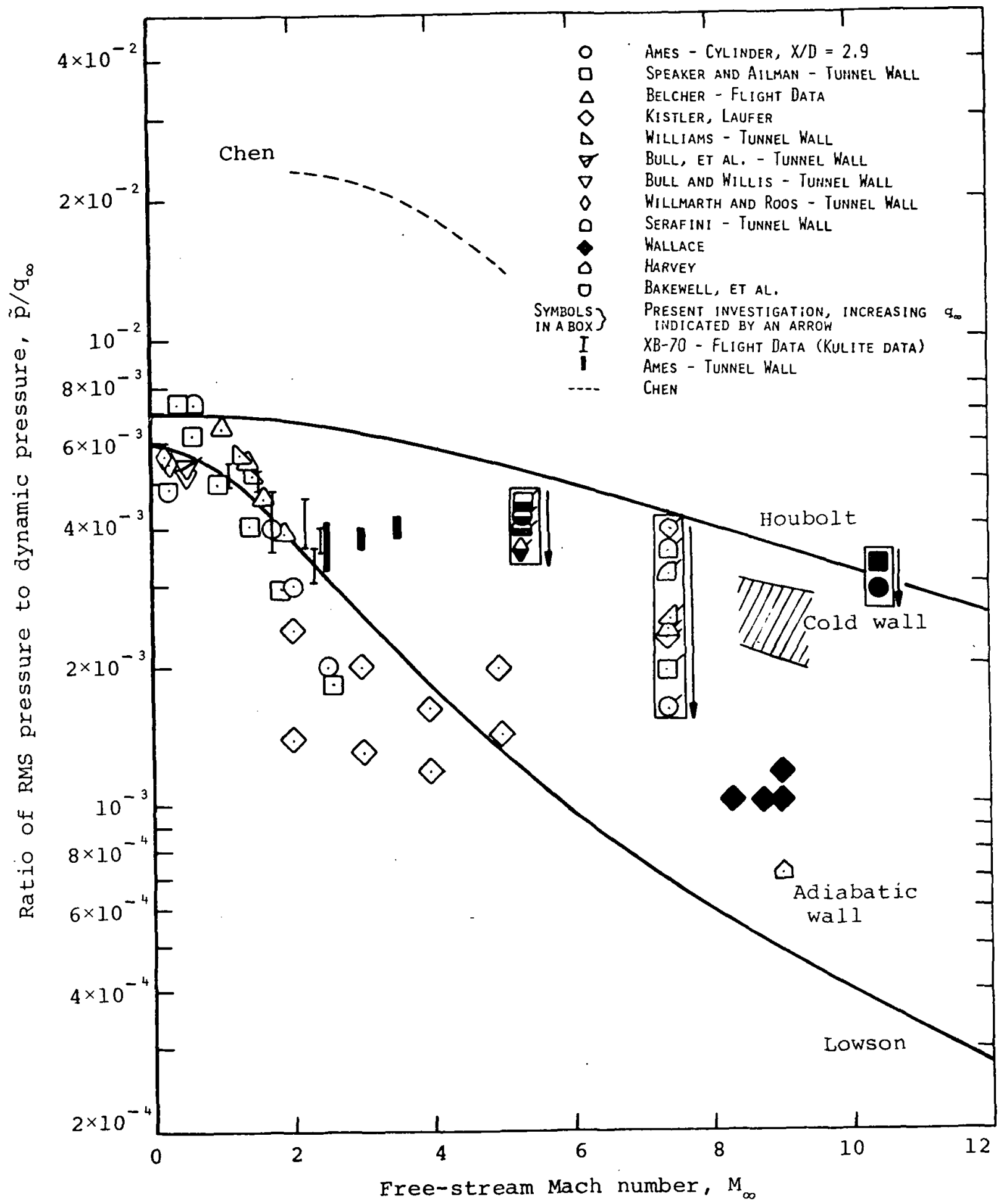

Figure 28.- Ratio of RMS pressure to dynamic pressure plotted against Mach number. 


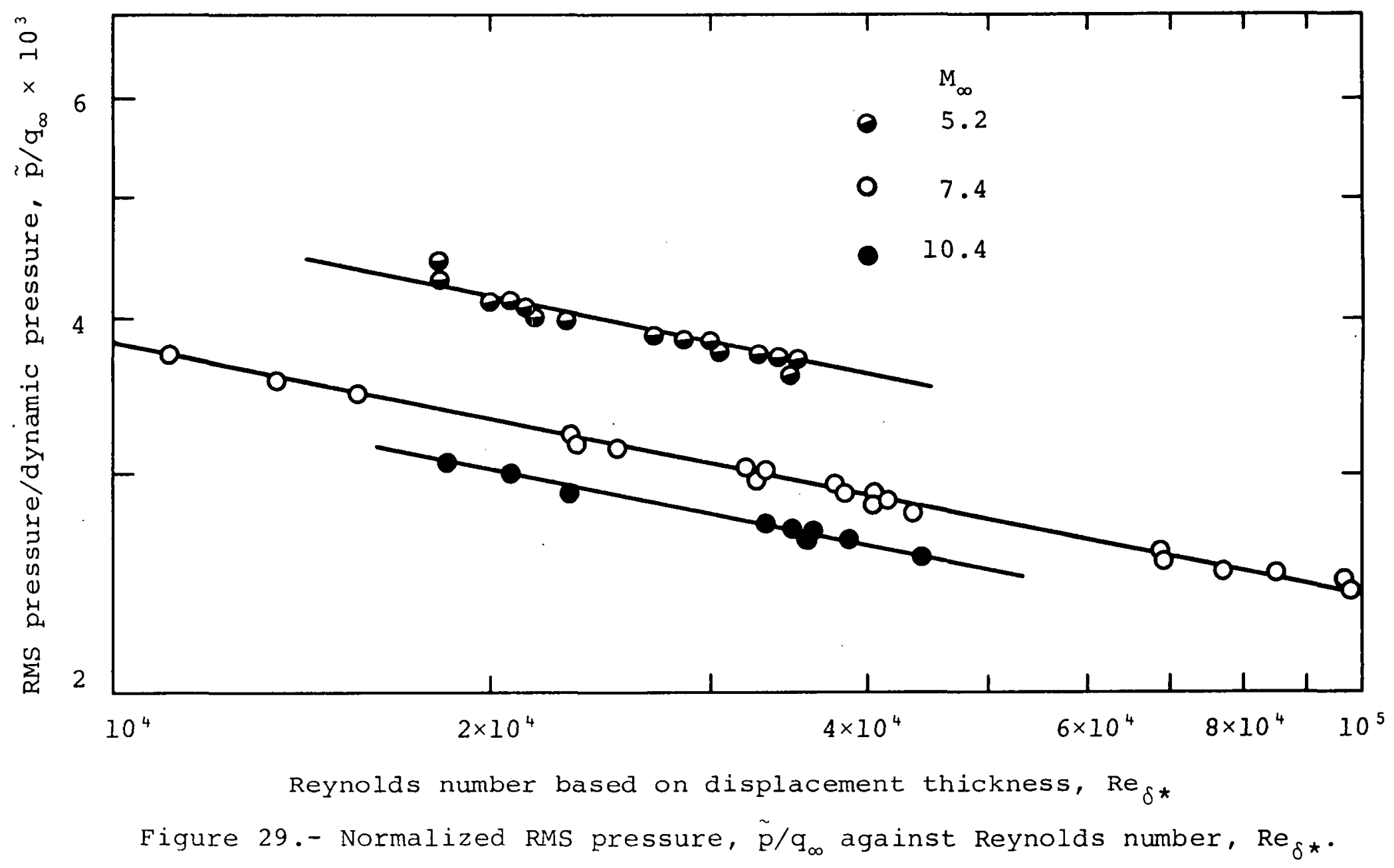




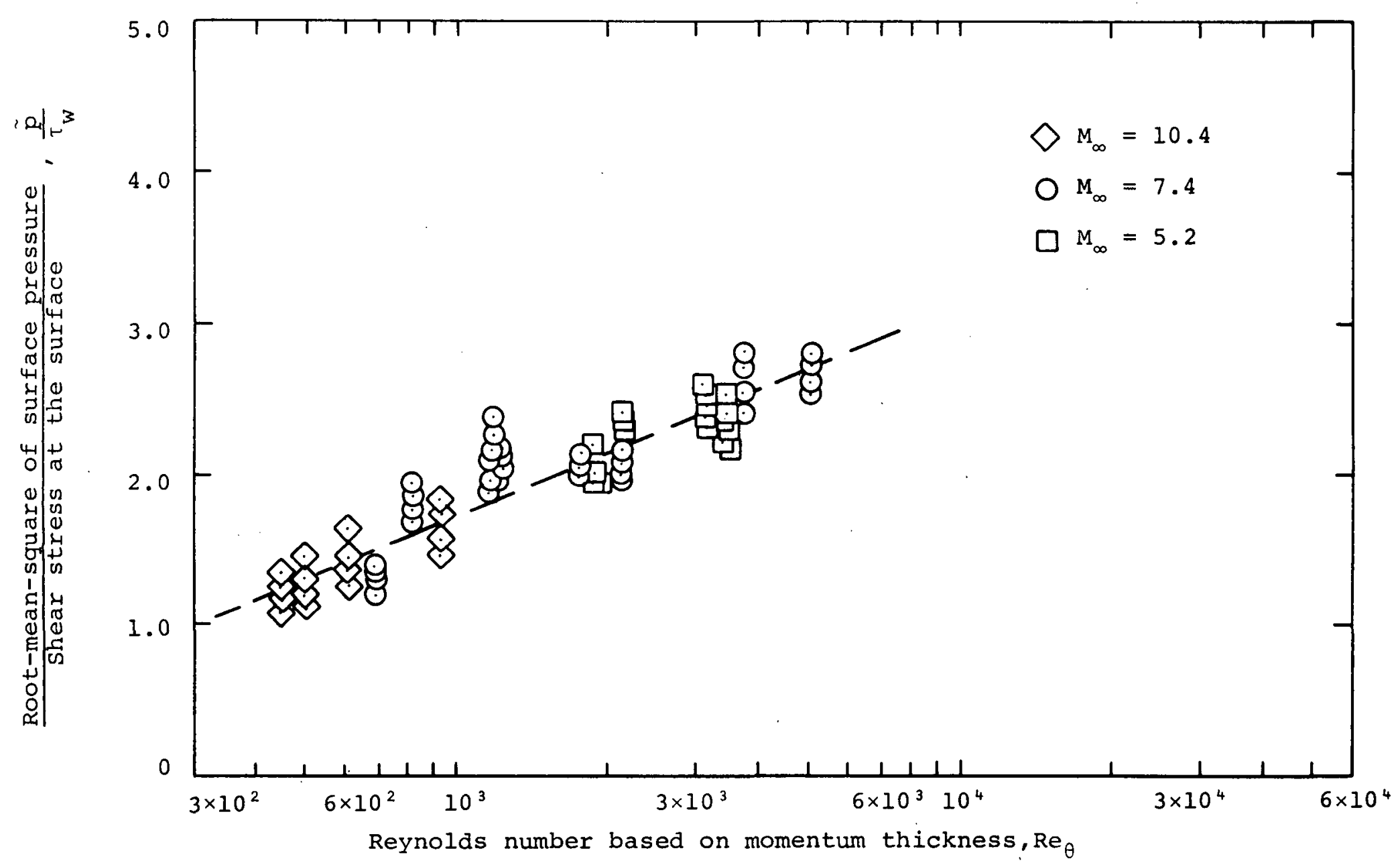

Figure 30.- Ratio of RMS pressure to local wall shear stress against Reynolds number, $\operatorname{Re}_{\theta}$. 


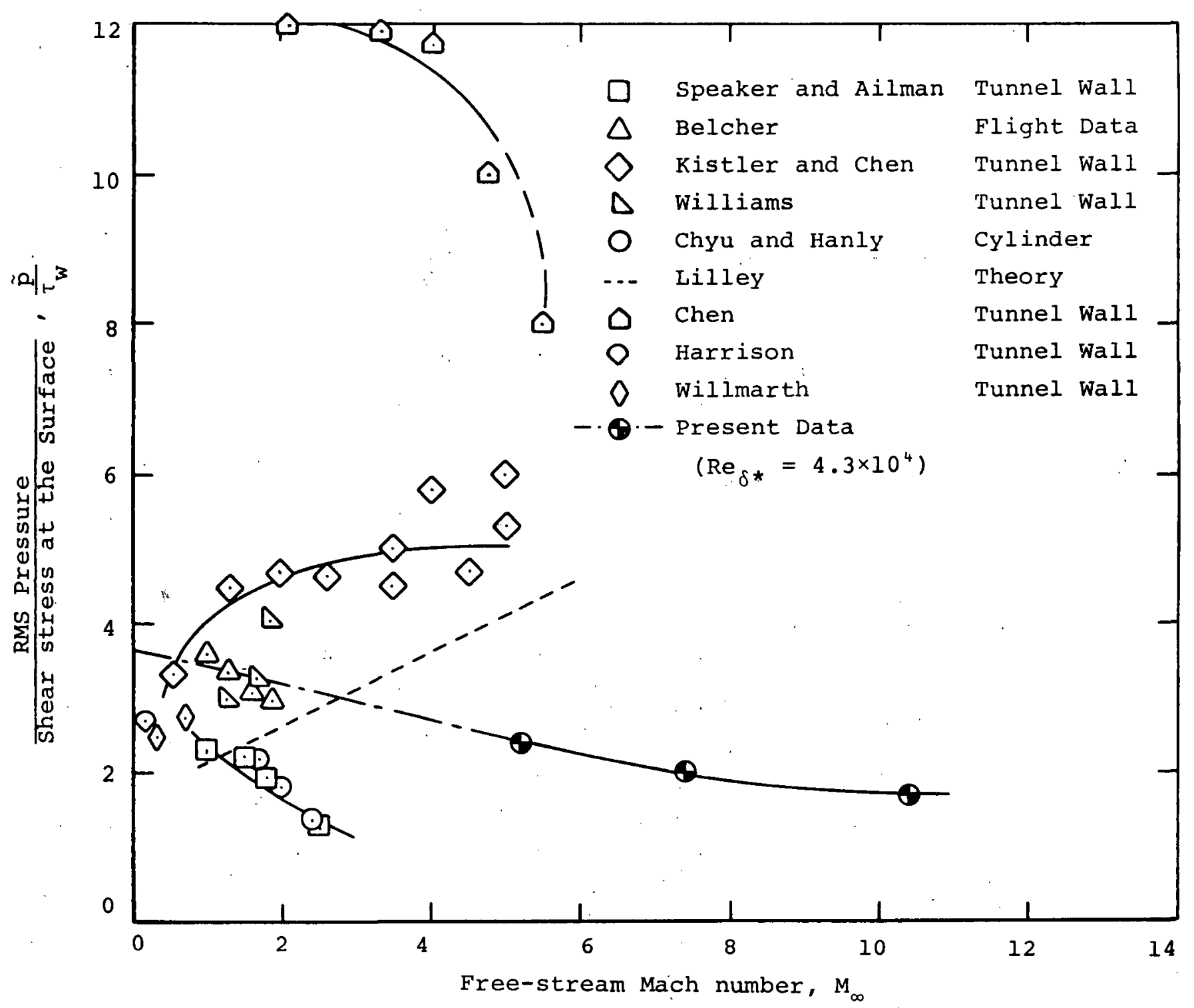

Figure 31.- Ratio of RMS pressure to local wall shear stress against Mach number, $M_{\infty}$. 

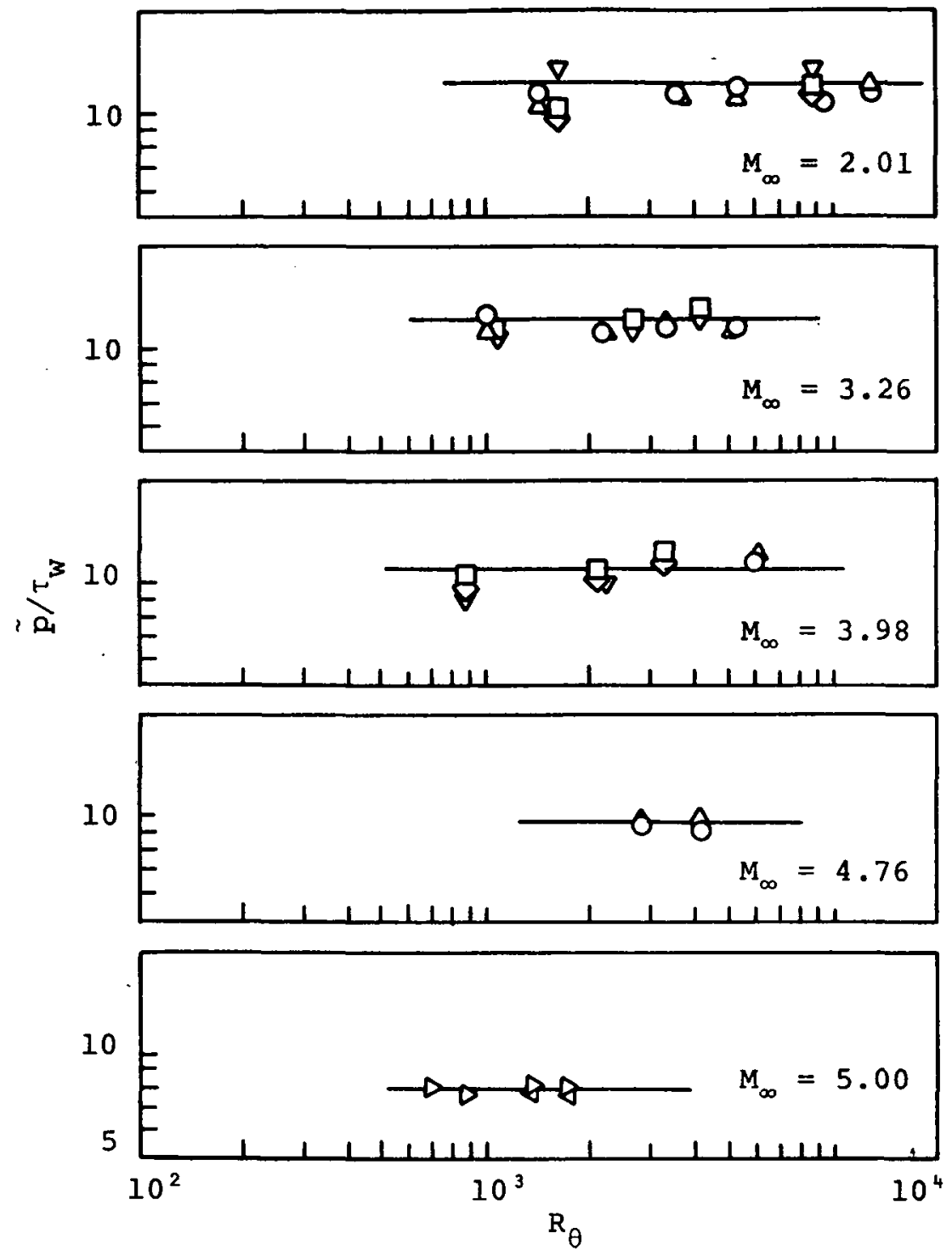

\begin{tabular}{|cc|c|c|}
\hline \multicolumn{2}{|c|}{ CRYSTAL } & D, IN & STATION \\
\hline O & A & 0.015 & 24 \\
$\Delta$ & B & 0.030 & 24 \\
0 & 4 & 0.015 & 13 \\
$\square$ & 5 & 0.015 & 13 \\
$\nabla$ & 6 & 0.030 & 13 \\
$D$ & 7 & 0.030 & 13 \\
$\square$ & 8 & 0.030 & 13 \\
\hline
\end{tabular}

Figure 32.- Data from reference $21-\tilde{p} / \tau_{w}$ against $\mathrm{Re}_{\theta}$, for flat-plate. 


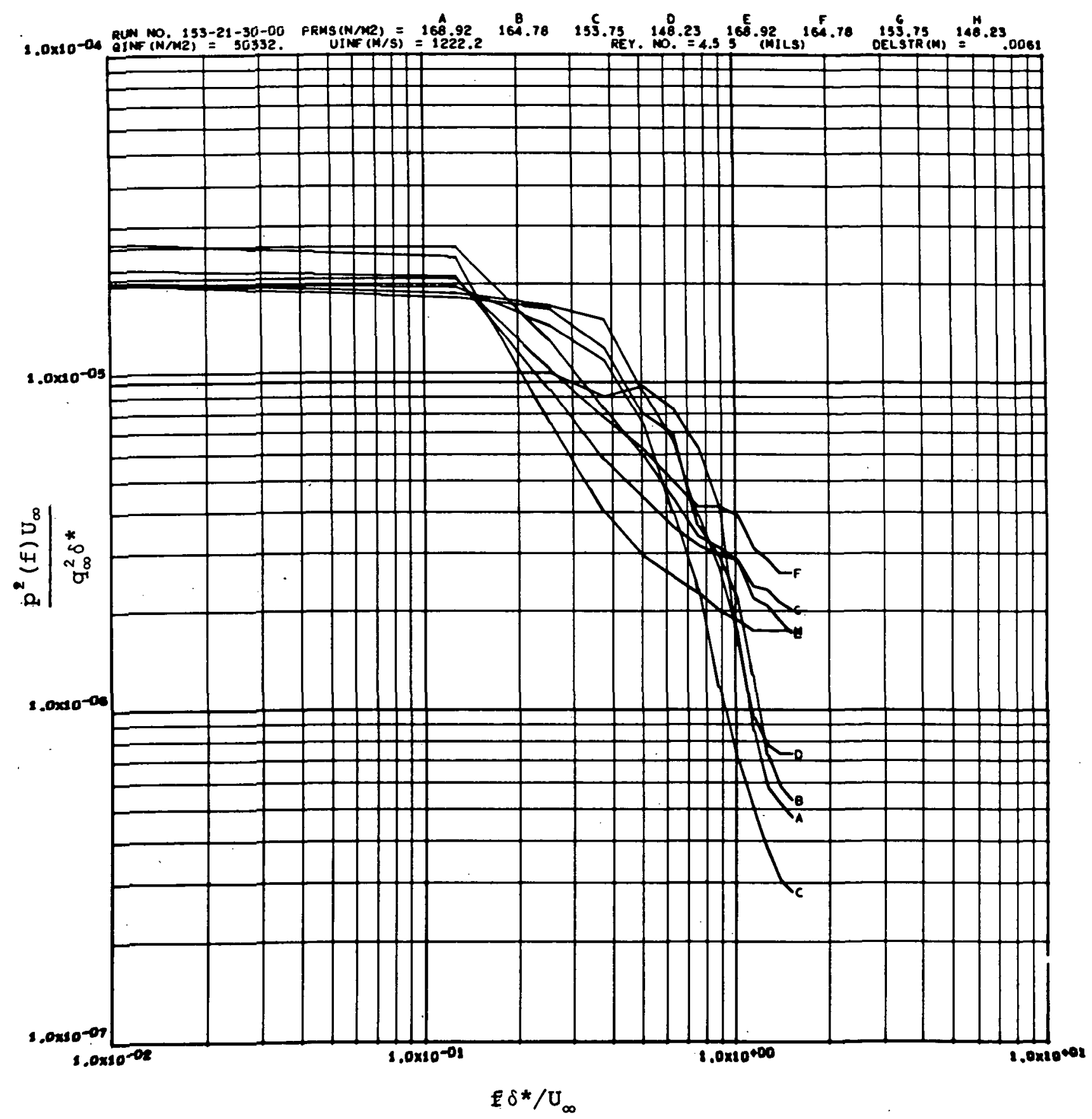

(a) $\mathrm{q}_{\infty}=50332 \mathrm{~N} / \mathrm{m}^{2}$ and $\cdot \mathrm{Re}_{\mathrm{X}}=4.55 \times 10^{6}$.

Figure 33.- Power spectrum of pressure fluctuations, $M_{\infty}=5.2$. 


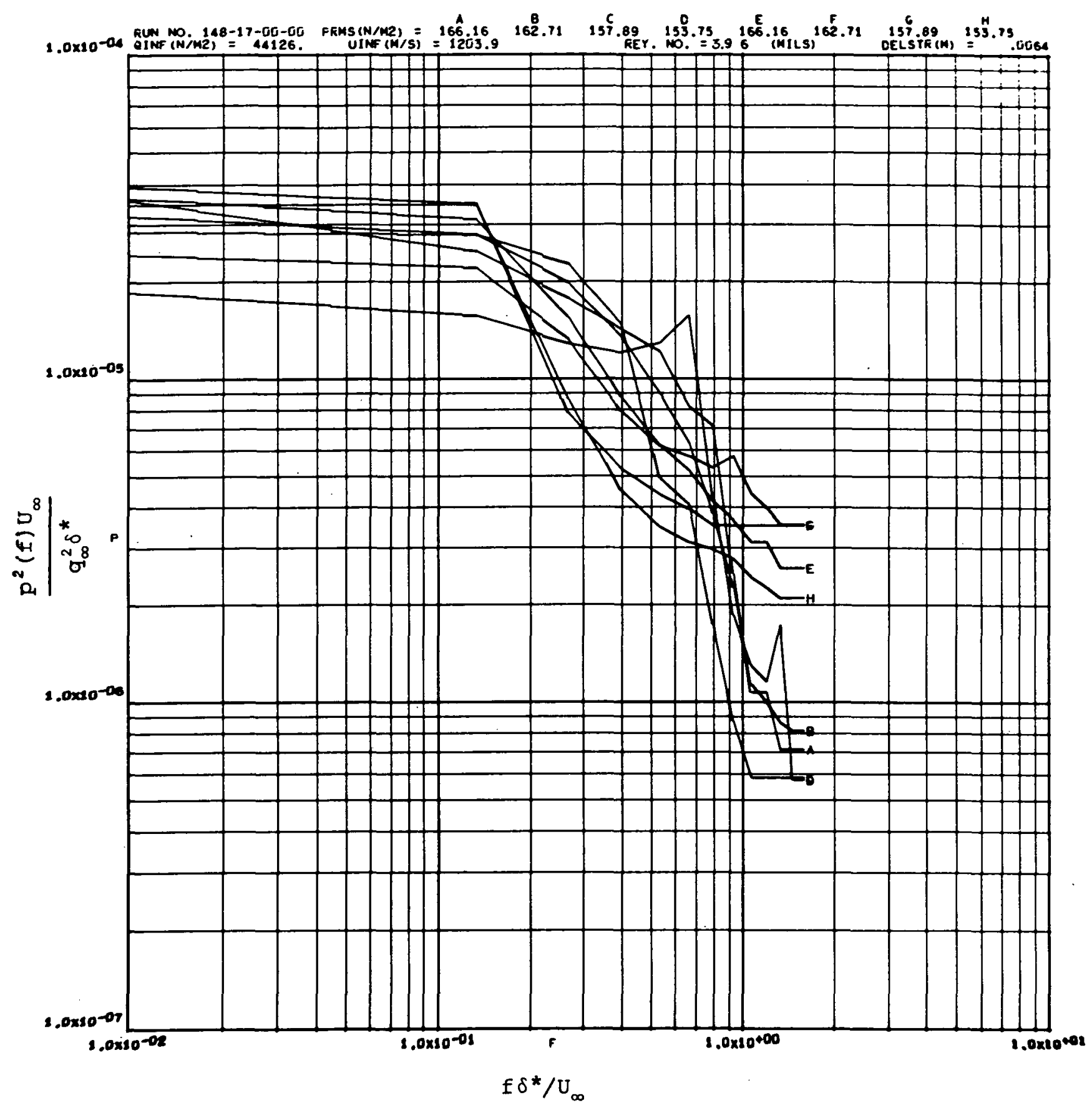

(b) $\mathrm{q}_{\infty}=44126 \mathrm{~N} / \mathrm{m}^{2}$ and $\operatorname{Re}_{\mathrm{x}}=3.96 \times 10^{6}$.

Figure 33.- Continued. 


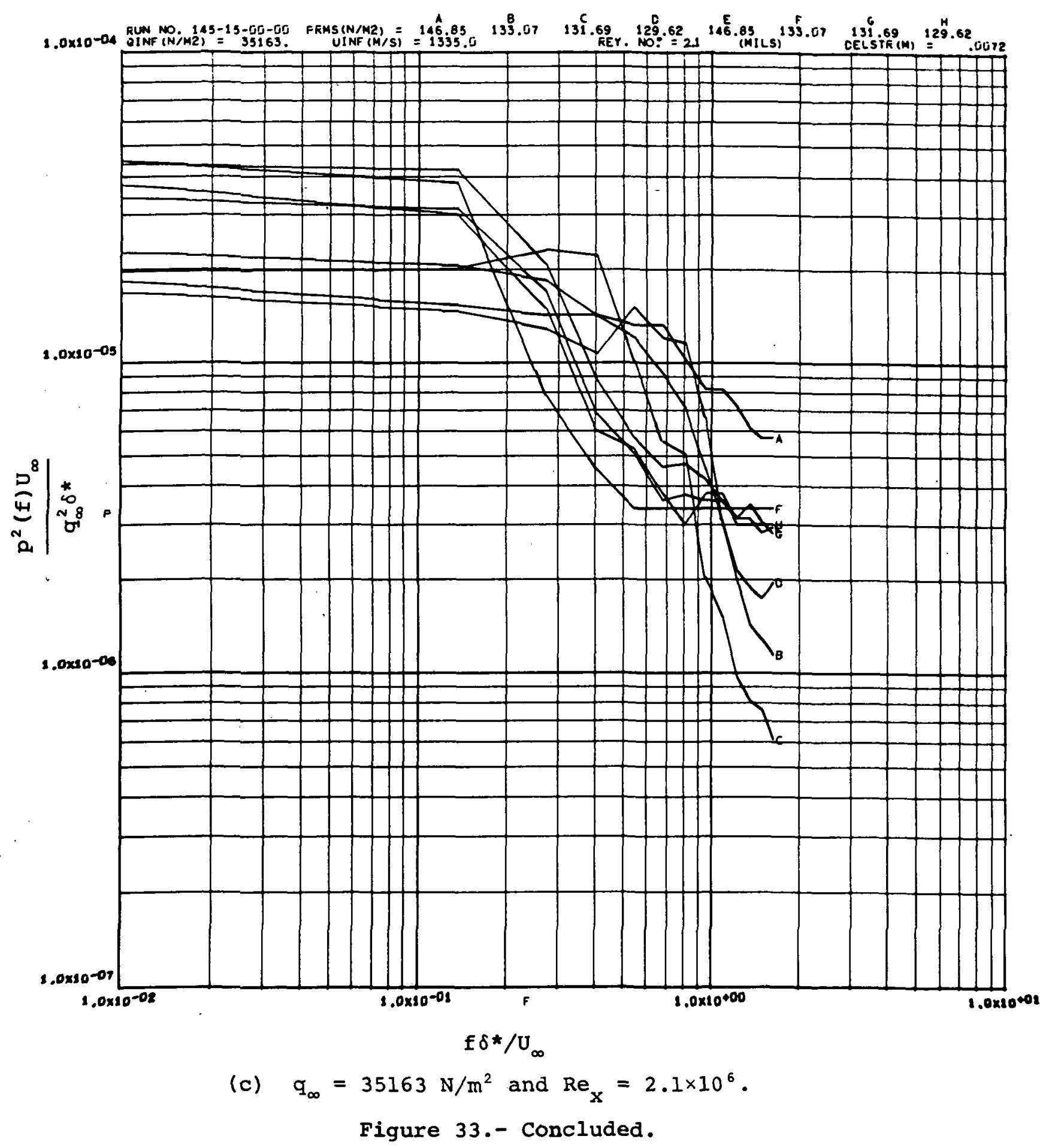




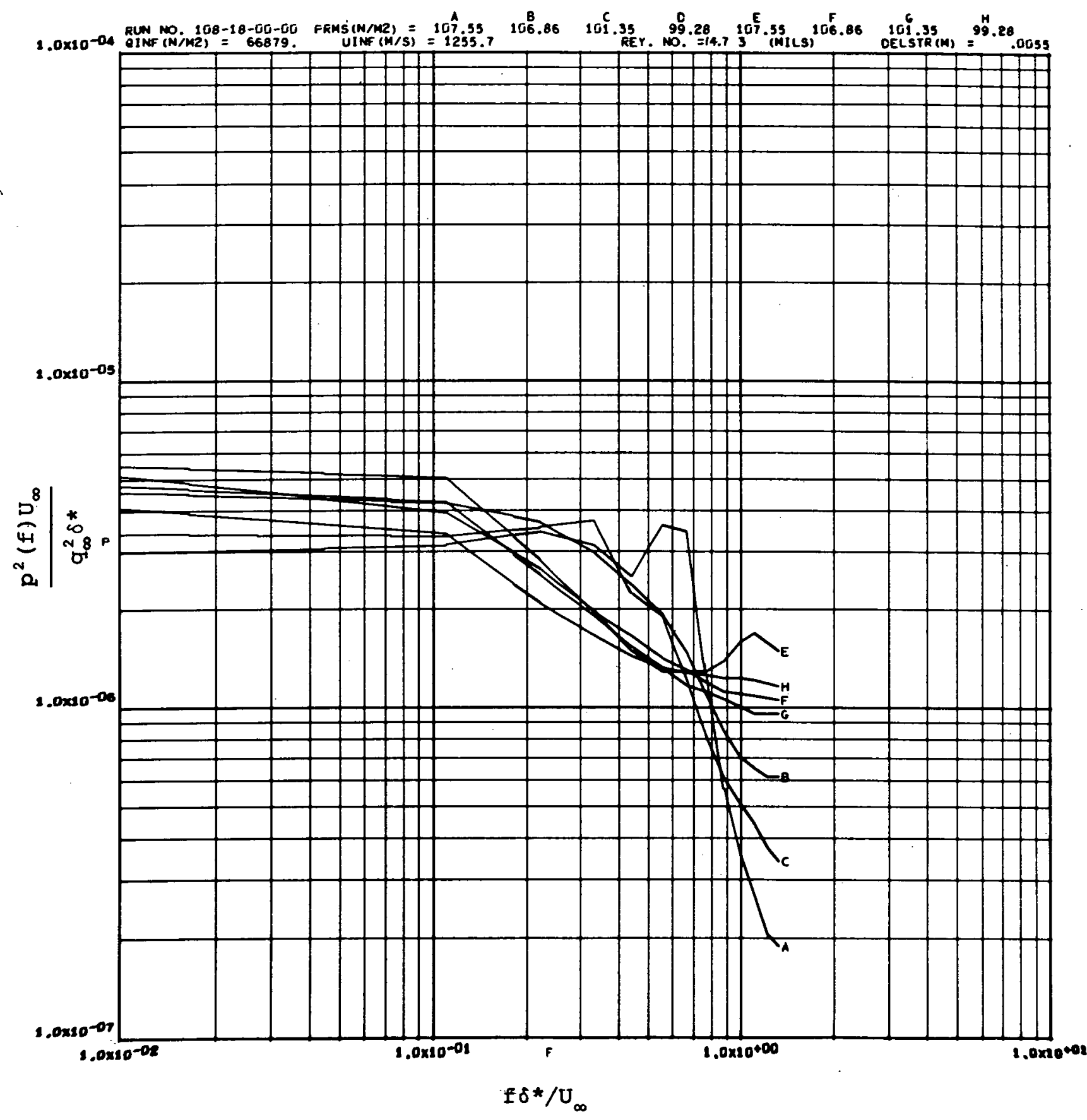

(a) $\mathrm{q}_{\infty}=66879 \mathrm{~N} / \mathrm{m}^{2}$ and $\mathrm{Re}_{\mathrm{x}}=14.73 \times 10^{6}$.

Figure 34.- Power spectrum of pressure fluctuations, $M_{\infty}=7.4$. 


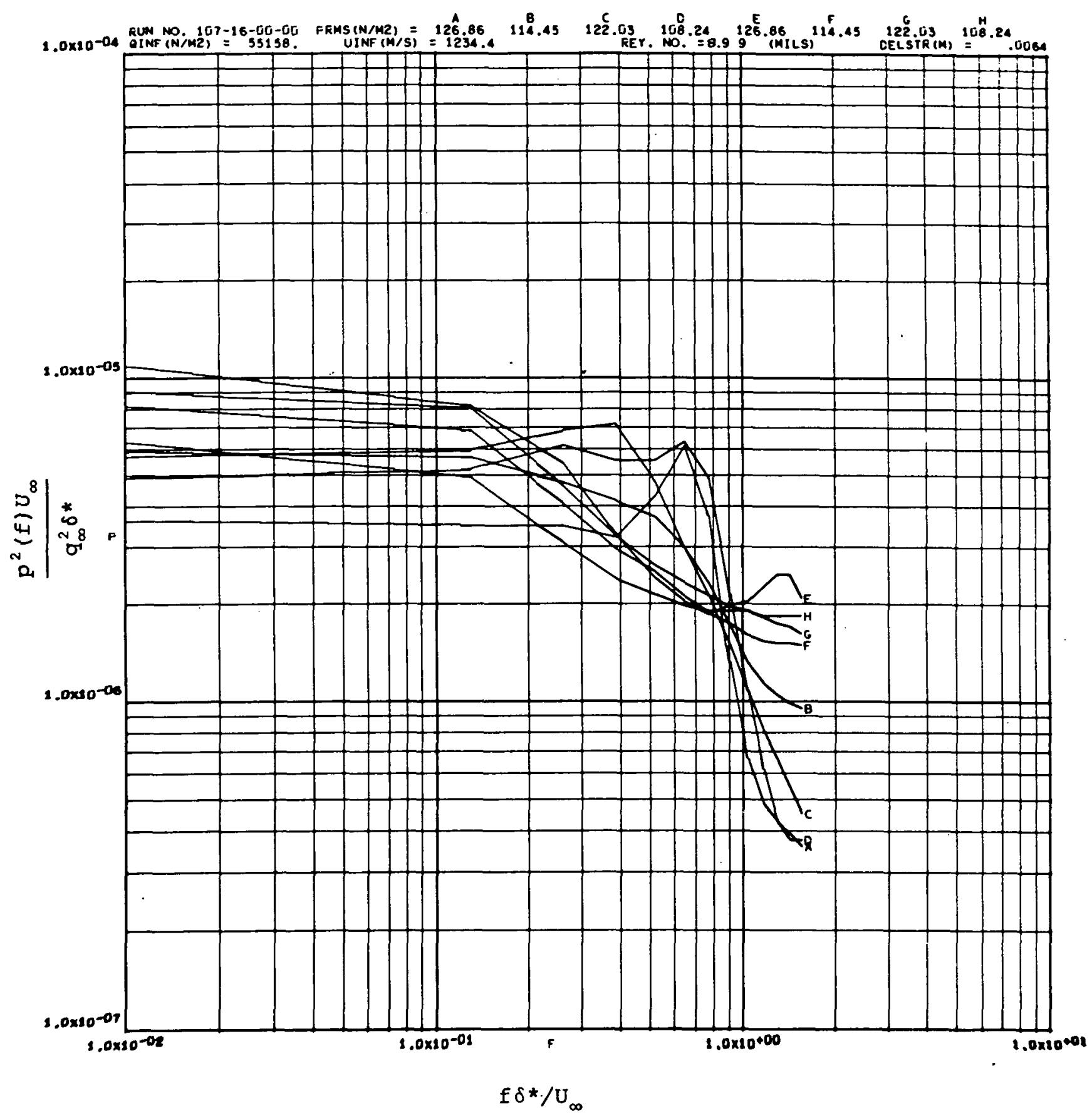

(b) $q_{\infty}=55158 \mathrm{~N} / \mathrm{m}^{2}$ and $R e_{x}=8.99 \times 10^{6}$.

Figure 34.- Continued. 


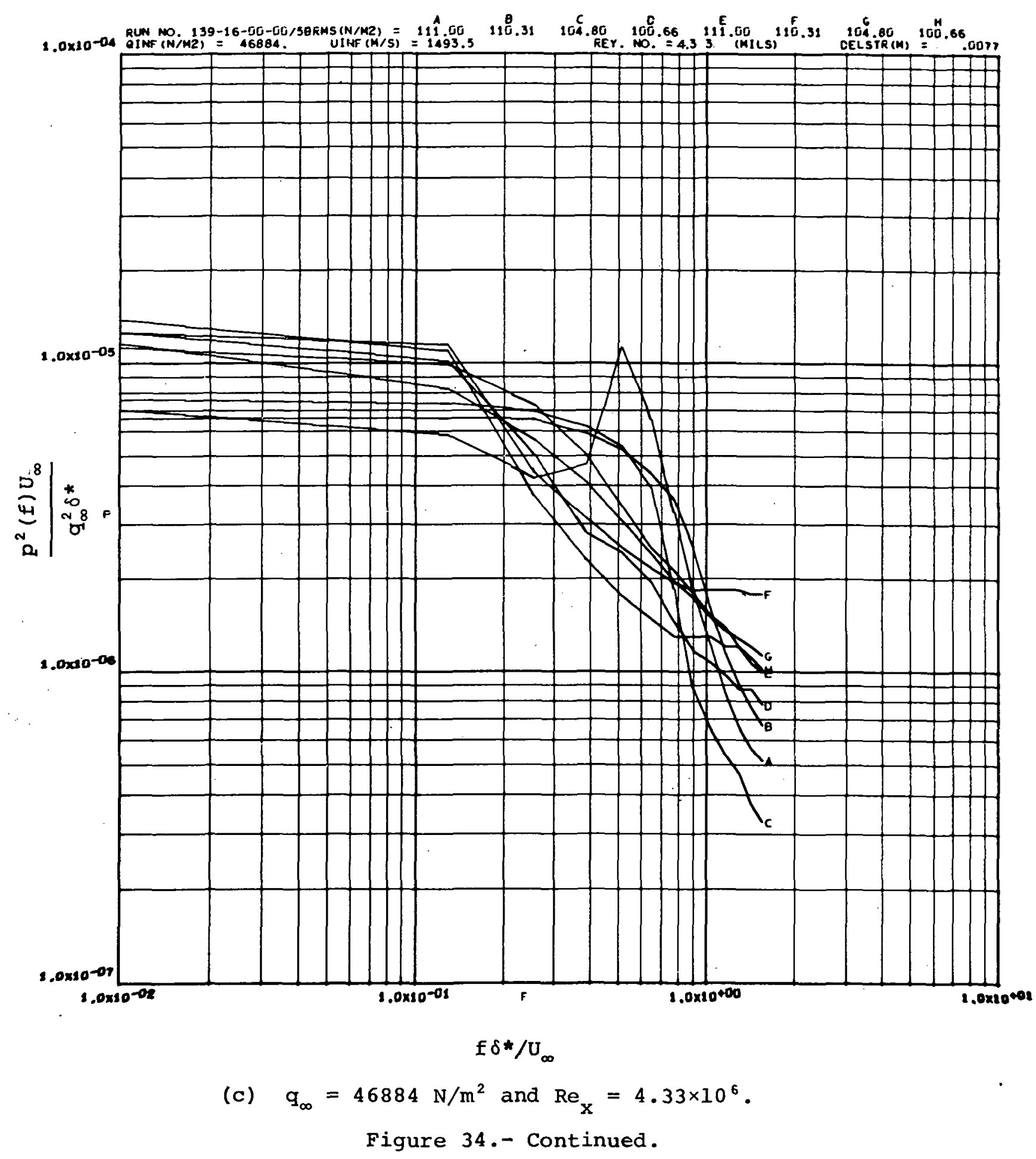




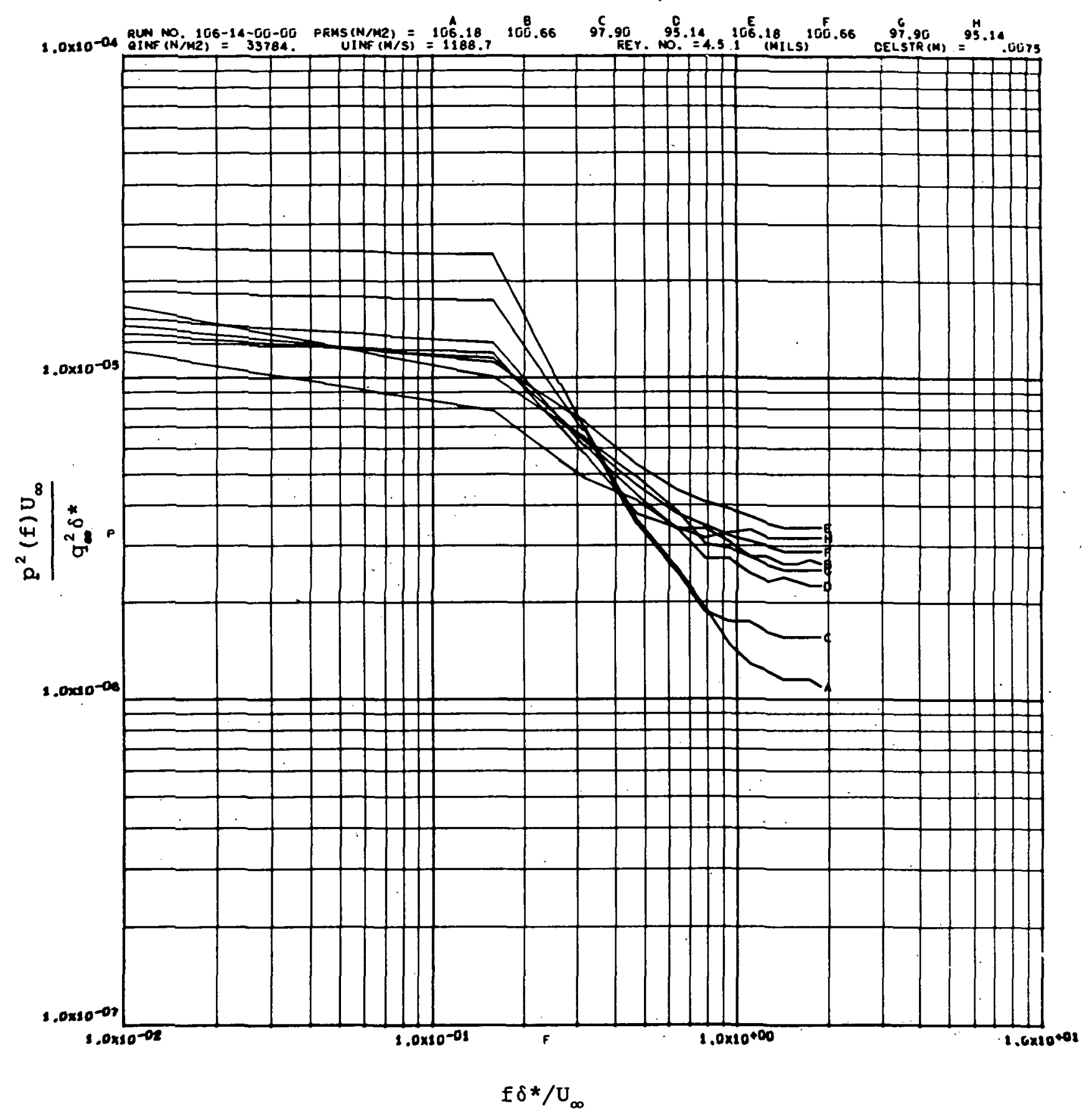

(d) $\mathrm{q}_{\infty}=33784 \mathrm{~N} / \mathrm{m}^{2}$ and $\mathrm{Re}_{\mathrm{x}}=4.51 \times 10^{6}$.

Figure 34.- Continued. 


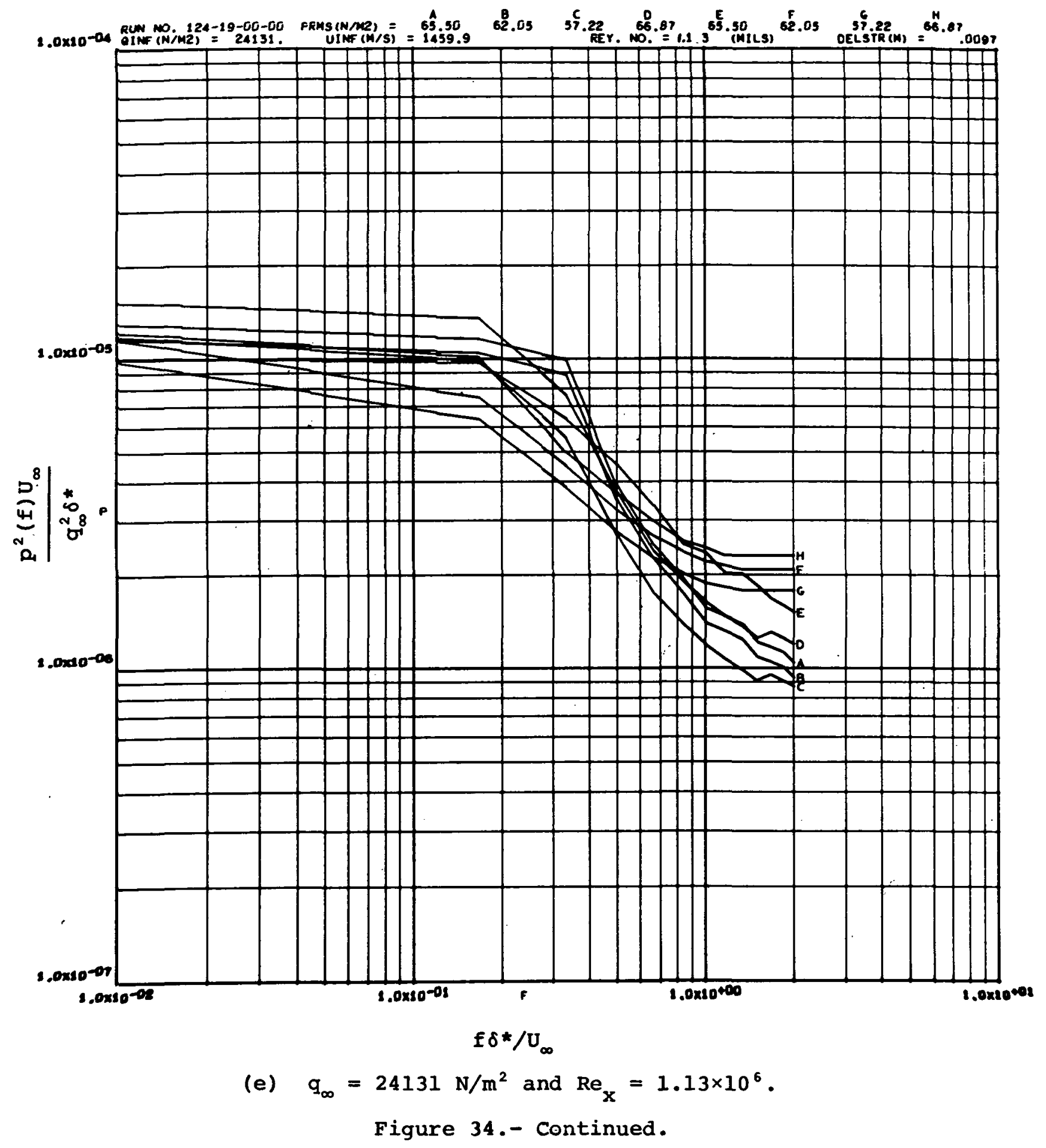




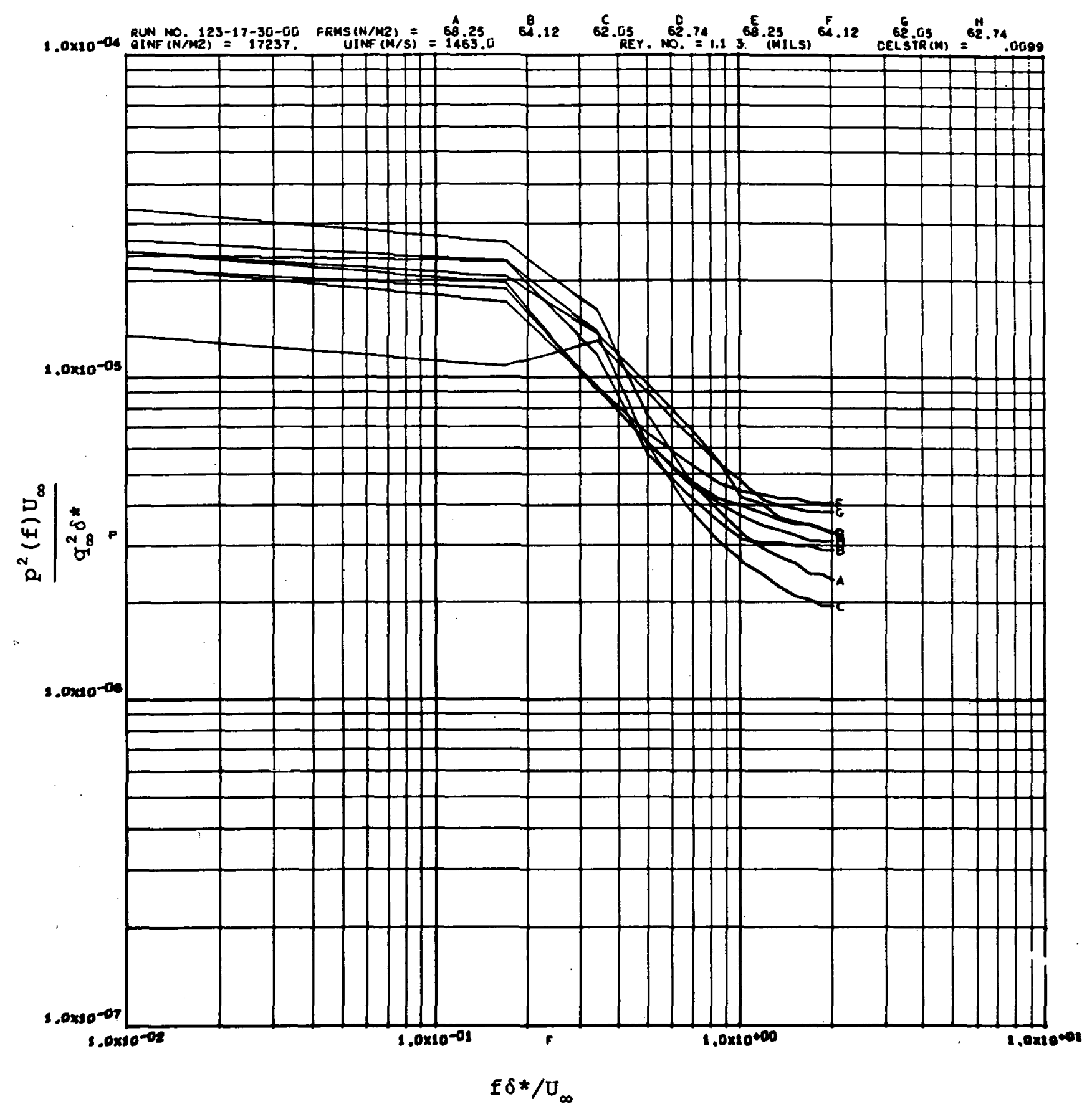

(f) $q_{\infty}=17237 \mathrm{~N} / \mathrm{m}^{2}$ and $R e_{X}=1.13 \times 10^{6}$. Figure 34.- Concluded. 


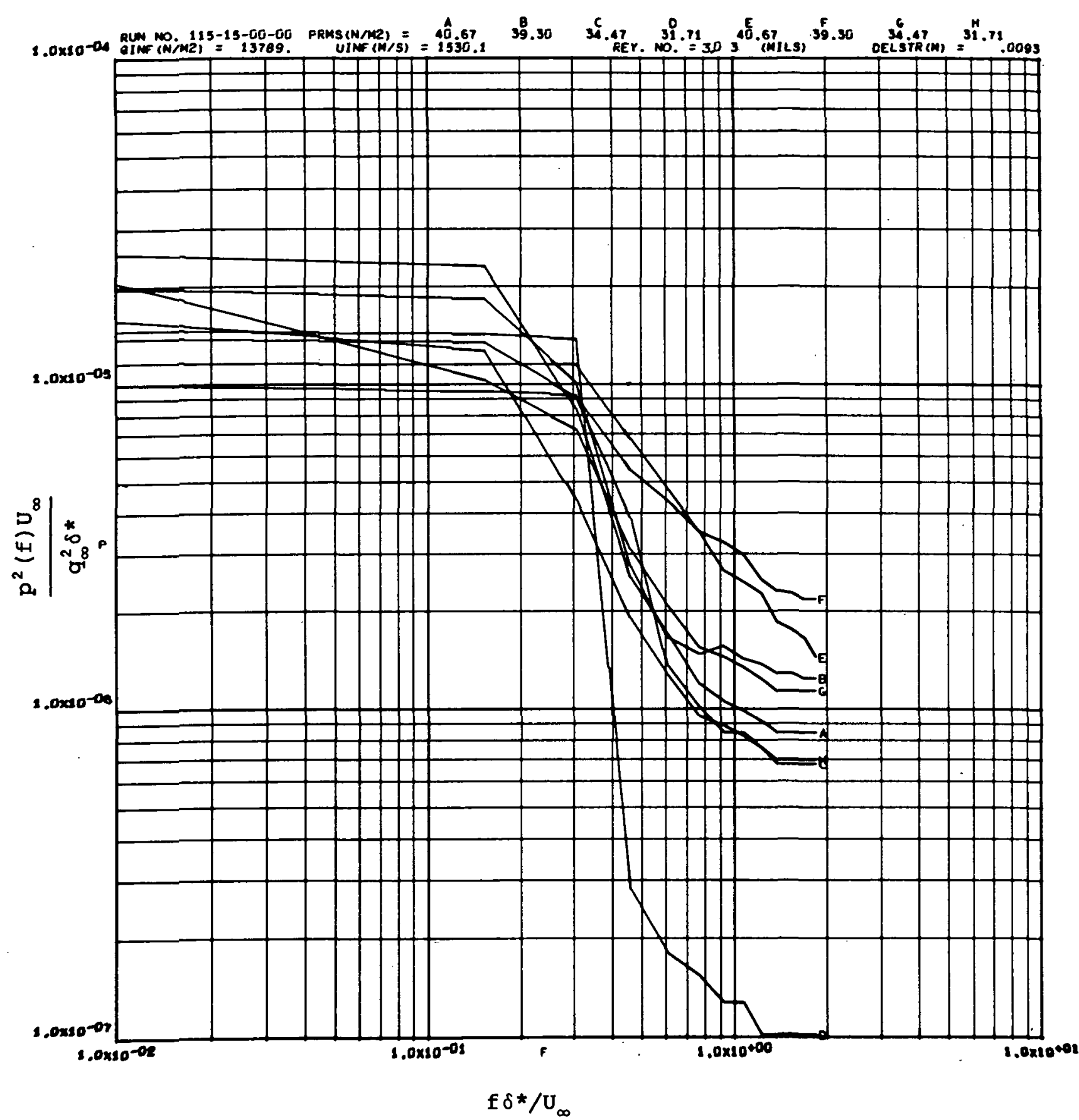

(a) $q_{\infty}=13789 \mathrm{~N} / \mathrm{m}^{2}$ and $R e_{X}=3.03 \times 10^{6}$.

Figure 35.- Power spectrum of pressure fluctuations, $M_{\infty}=10.4$. 


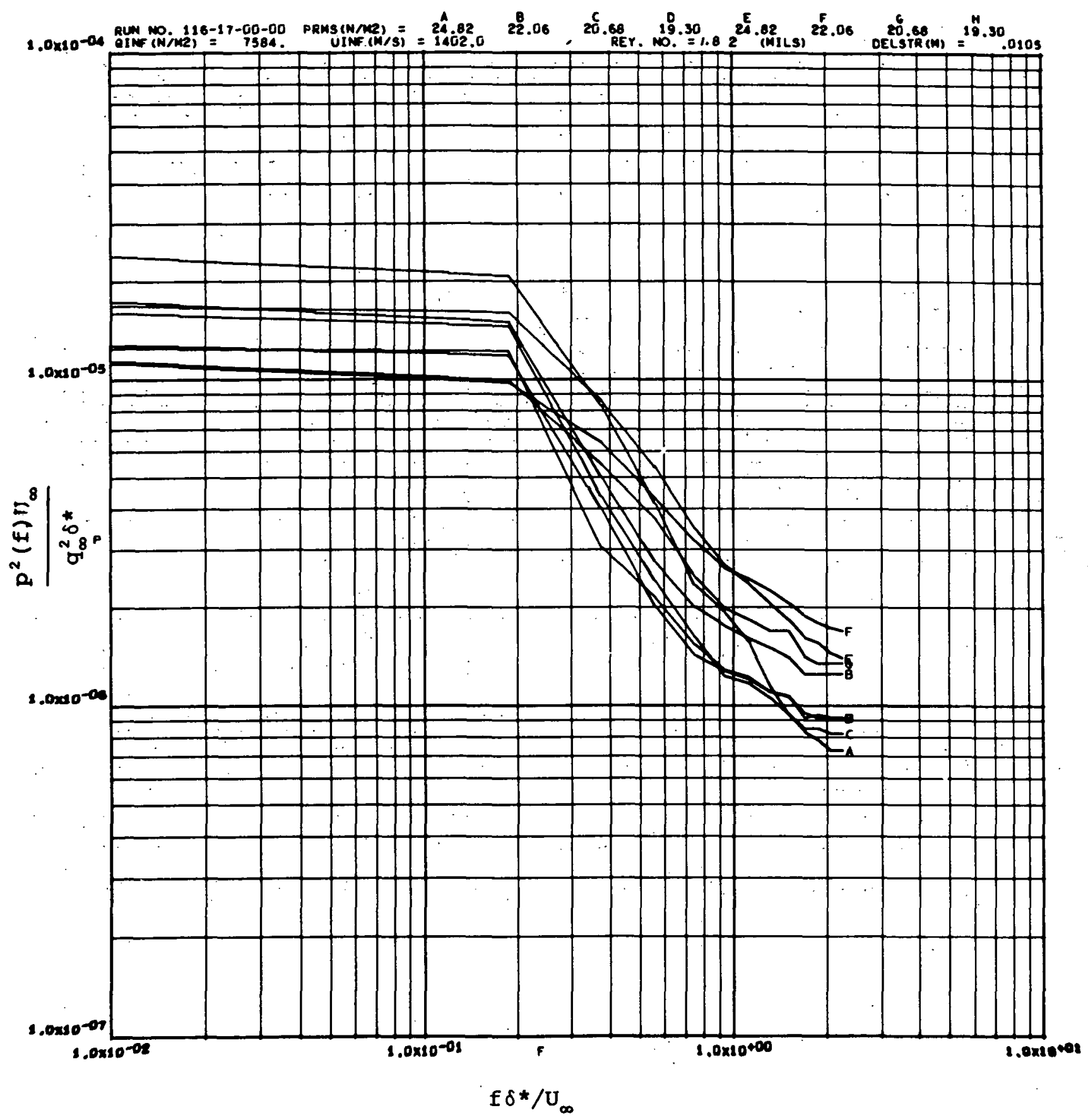

(b) $q_{\infty}=7584 \mathrm{~N} / \mathrm{m}^{2}$ and $\operatorname{Re}_{\mathrm{x}}=1.82 \times 10^{6}$.

Figure 35.- Continued. 


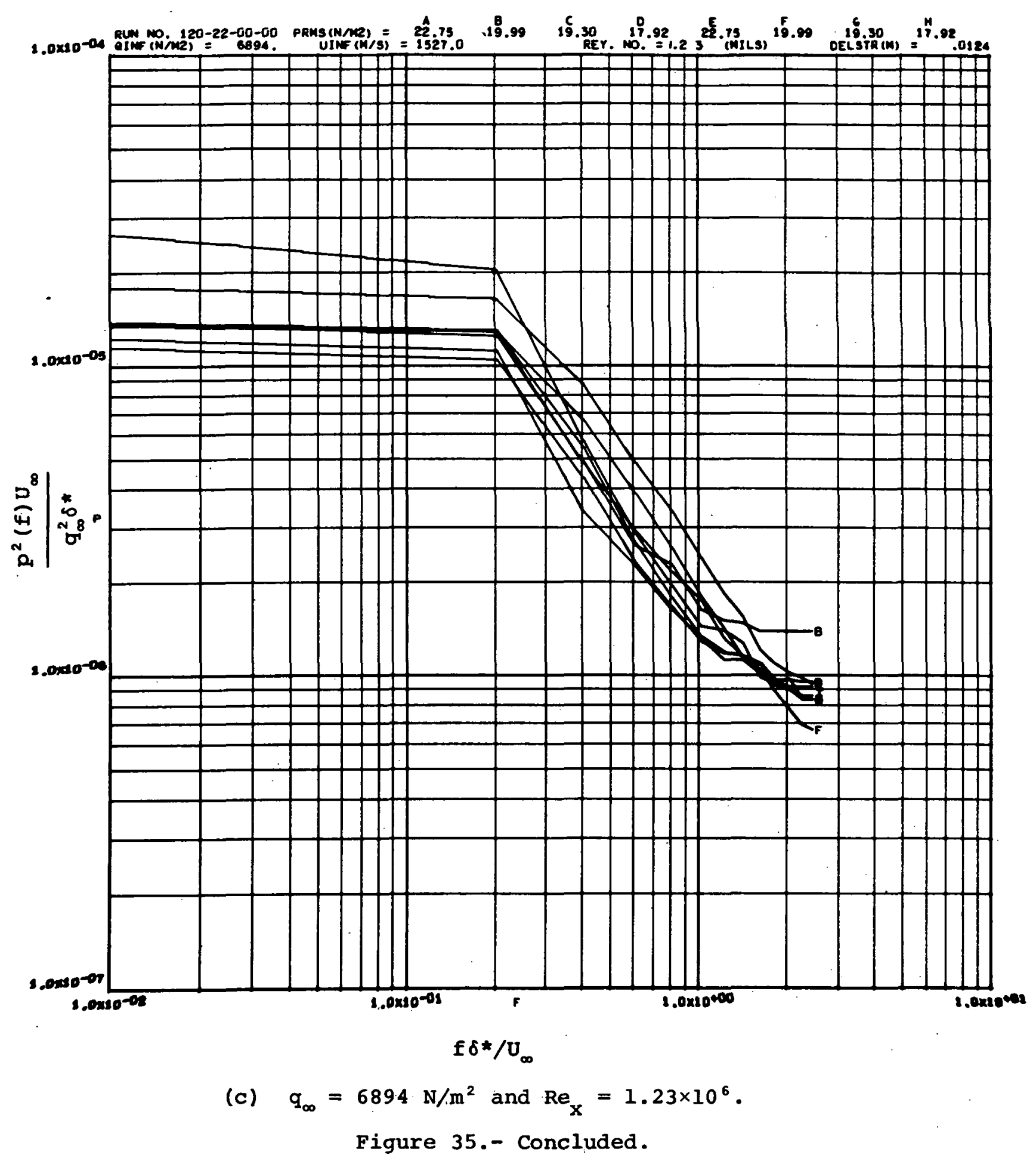




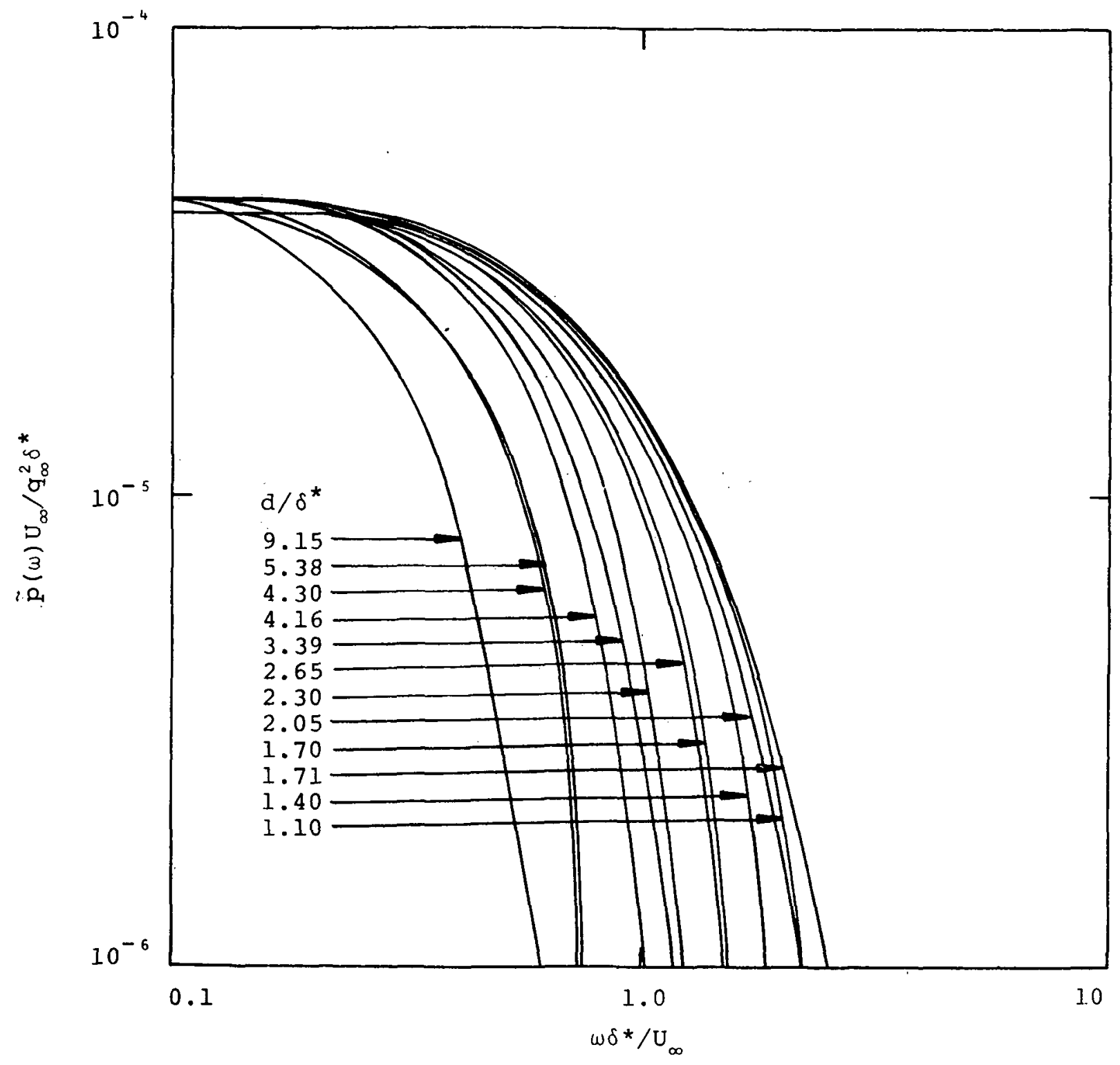

Figure 36.- Replot of Willmarth and Serafini's data. 


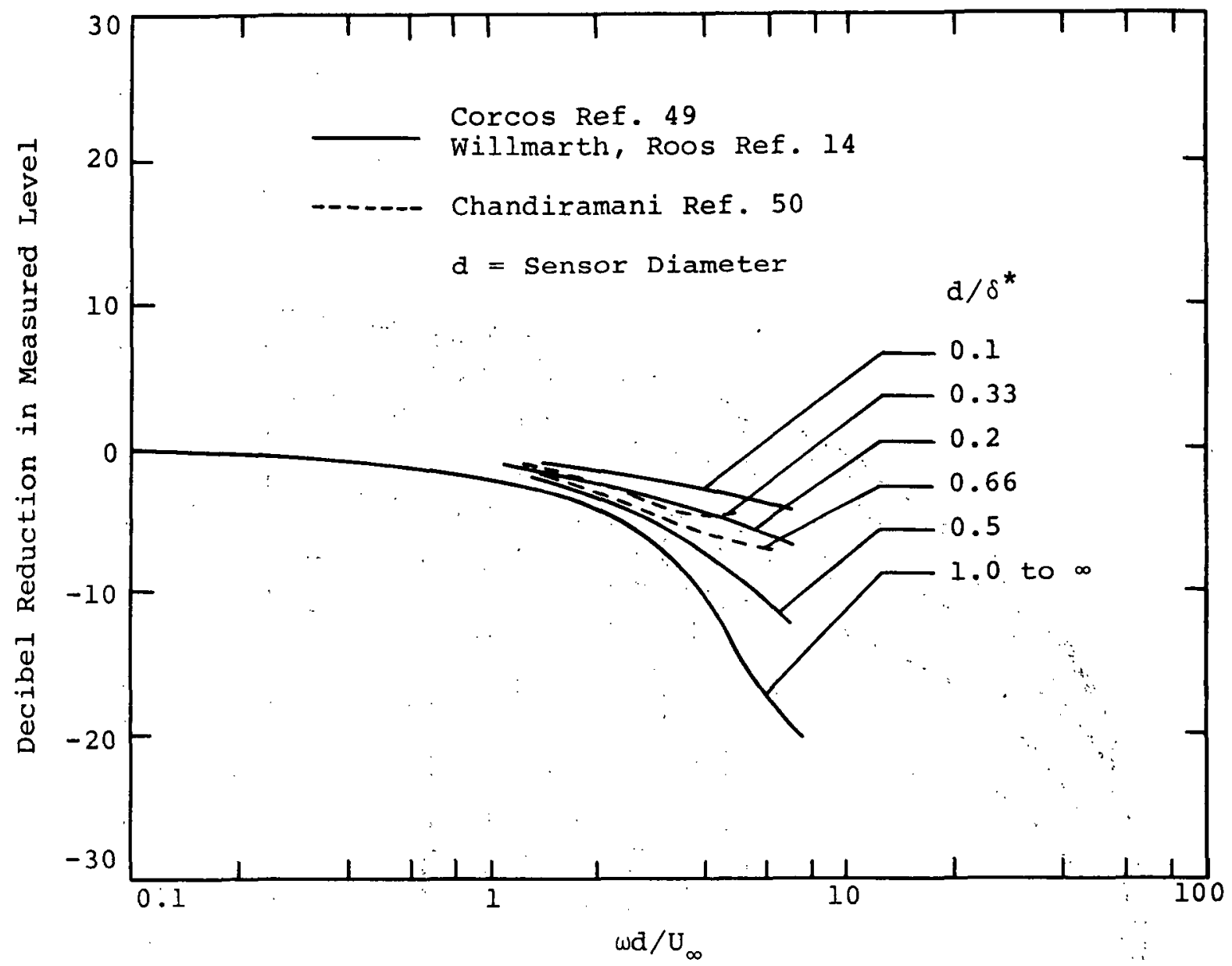

Figure 37.- Transducer size and its effects on frequency response. 

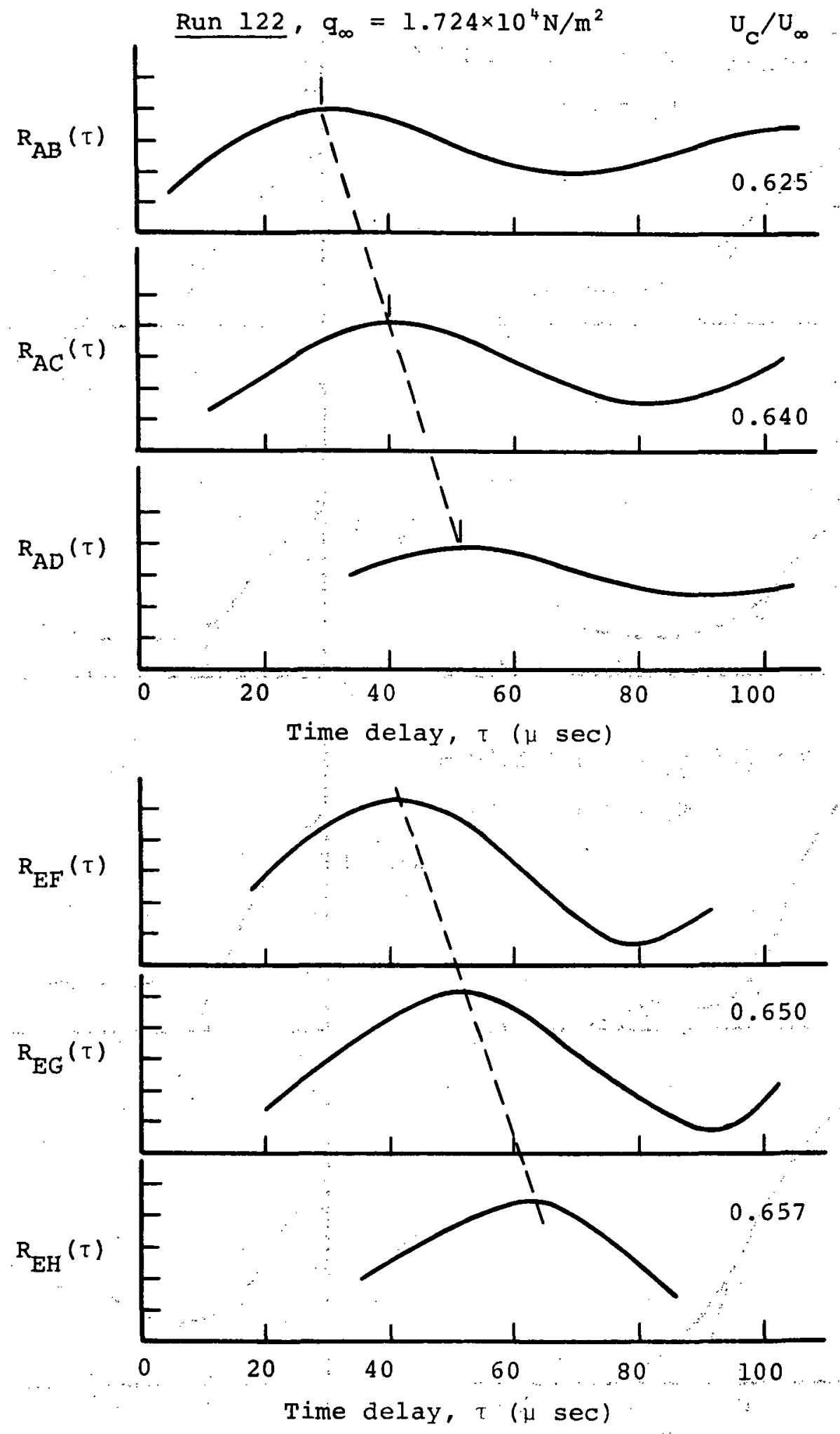

Figure 38.- Space-time correlation for $M_{\infty}=7.4$. 

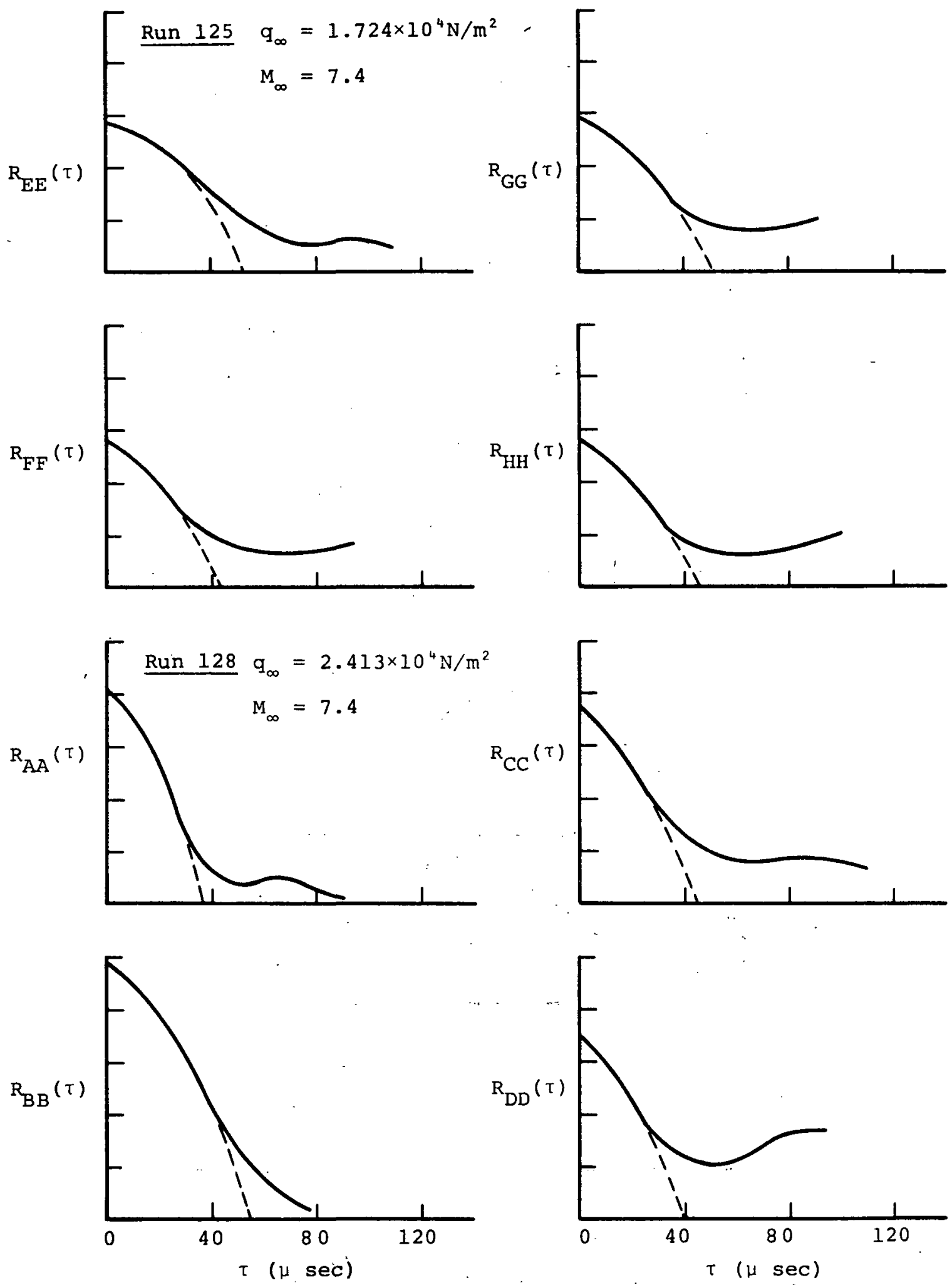

Figure 39.- Auto correlations. 